Visual Regulation of Manual Aiming 


\title{
VISUAL REGULATION OF MANUAL AIMING
}

\section{By}

ROMEO C. CHUA

\begin{abstract}
A Thesis
Submitted to the School of Graduate Studies in Partial Fulfillment of the Requirements for the Degree Master of Science McMaster University
\end{abstract}

(c) Copyright by Romeo C. Chua, June 1992 
MASTER OF SCIENCE (1992)

MCMASTER UNIVERSITY

(Human Biodynamics)

Hamilton, Ontario

TITLE: Visual Regulation of Manual Aiming

AUTHOR: Romeo C. Chua, B.Sc.(Kinesiology)

(Simon Fraser University).

SUPERVISOR: Dr. Digby Elliott

NUMBER OF PAGES: ix, 188 


\begin{abstract}
Traditional models of visuomotor control have generally emphasized the importance of vision in the guidance of limb movements. Vision is thought to subserve the modificational processes underlying the control of these movements. The objectives of the present work were to elaborate upon the role of vision in the regulation of an ongoing limb movement, address issues pertaining to the nature of this regulation, and examine predictions of the Optimized Submovement Model (Meyer, Abrams, Kornblum, Wright, \& Smith, 1988) of limb control. An aiming task was adopted in which subjects were required to displace a graphics cursor on a monitor toward a target. The presence of visual feedback proved to be a potent determinant of performance. In Experiment 1, superior performance consistency with visual feedback was attributable to the prevalence of discrete and continuous modifications made to the movement when visual information was available. In Experiment 2, the same visually-based performance advantage was found. However, this advantage was no longer related to the presence of adjustments to the movement. The present results are discussed with reference to current issues in the nature of visuomotor regulation and their implications toward the Optimized Submovement Model.
\end{abstract}




\section{Acknowledgments}

There are a number of people to whom I wish to extend my sincere gratitude.

Heather Carnahan, Jim Dowling, and Janet Starkes, for serving as members of my committee and providing me with the benefits of their expertise

Brian Maraj, Barb Pollock, Shannon Robertson, and Laurie Swanson, for their friendship and support.

Joe, Tricia, and Alexa Winik, for being my family away from home.

Richard Carson, for the valuable lessons from the work we have done and all that fun with the Touchscreen

David Goodman, for providing me with the opportunity to work in the lab while completing my undergraduate degree

Digby Elliott, for his teachings, guidance, advise, enthusiasm, and friendship I could not have asked for a better advisor Thanks for always leaving your door open, Dig

Brenda and my family, for their love and encouragement 
Table of contents

Descriptive Note ii

Abstract iii

Acknowledgements iv

List of Tables. vii

List of Figures. viii

Introduction 1

Woodworth (1899): A Beginning 2

Fitts' LaW

Iterative Corrections Model

Stochastic optimized Dual Submovement Model 11

Visual-Motor Control and the optimized Dual

Submovement Model

Optimized Dual Submovement Model 15

Contribution of Vision During the Early and

Latter Stages of Aiming Movements.

Modificational Role of Vision and the Nature of

Visual-Motor Regulation 25

Examination of the Role of Vision in Manual Aiming and of the Stochastic Optimized Dual Submovement Model 32

Experiment 1 34

Method 37

Subjects. 37

Apparatus 37

Procedure. 39

Data Reduction 45

Movement Modifications 46 Results 56

Performance Measures 56

Kinematic Measures 60

Correlational Analyses 67 
Discussion 68

Experiment 2 78

Method 80

Subjects 80

ApparatUS 81

Procedure

Data Reduction 84

Results 84

Performance Measures $S_{\text {ans }} 85$

Kinematic Measures _ _ 88

Correlational Analyses 93

Partial-Vision Conditions 94

Performance Measures 94

Kinematic Measures

Discussion 100

General Discussion 113

ReferenCeS 122

Appendix A

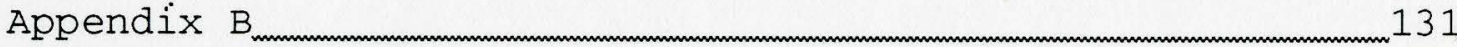

Appendix $C_{\text {n }} 153$ 


\section{List of Tables}

Table

Page

1 Experiment 1: Mean sum of zero crossings in the acceleration profile as a function of Vision condition and target size 


\section{List of Figures}

Figure

Page

1 Graph of results from Woodworth (1899) Tables I-IV 5

2 Diagram of graphics tablet mouse 40

3 Diagram of graphics tablet and computer monitor mumm 41

4 Definition of a significant deviation in the acceleration profile prior to time of peak velocity 48

5 Schematic of an acceleration profile with two significant deviations prior to time of peak velocity

6 Definition of significant deviation in the acceleration profile following time of peak velocity 51

7 Schematic of an acceleration profile with two significant deviations following time of peak velocity

8 Definition of a zero crossing from velocity and acceleration profiles 54

9 Definition of a reversal of direction of movement 55

10 Experiment 1: Movement time as a function of Vision condition and target size

11 Experiment 1: Variable error as a function of Vision condition and target size

12 Experiment 1: Time to peak velocity/Movement time as a function of Vision condition and target size 61

13 Experiment 1: Displacement at time of peak velocity/target distance as function of Vision condition and target size,

14 Experiment 1: Mean sum of significant deviations in the acceleration profile following time of peak velocity as a function of vision condition and target size 
15 Experiment 2: Constant error as a function of Vision condition

16 Experiment 2:Variable error as a function of Vision condition

17 Experiment 2: Time to peak velocity/Movement time as a function of Vision condition

18 Experiment 2: Displacement at time of peak velocity/ target distance as a function of Vision condition 92

19 Experiment 2: Constant error as a function of Partial-Vision condition and group type

20 Experiment 2: Time to peak velocity/Movement Time as a function of Partial-Vision condition and group type

21 Experiment 2: Displacement at time of peak velocity/target distance as a function of PartialVision condition and group type 
Introduction

Movements of the limb which require a reasonable degree of precision are usually performed much more slowly than those which have no such requirements. If one is to increase the speed of execution, it is often done at the expense of movement accuracy or finesse. Accuracy is compromised for speed. This speed-accuracy tradeoff has received considerable attention over the past century. In the search for the sources underlying the speed-accuracy tradeoff, interest in the role which vision plays in regulating movements have also emerged. Not only have scientists been interested in why movement accuracy suffers with increases in the speed of execution, but also in how visual information is used to control the movement.

Several theories or models have been forwarded to account for the speed-accuracy relation and the role of vision in the regulation of goal-directed movement (e.g., see Jeannerod, 1988; Meyer, Smith, Kornblum, Abrams, \& Wright, 1990, for reviews). Many of these models have emphasized the importance of visual information for the accurate guidance of limb movements. Vision has been the primary modality thought to subserve modificational processes in the control of these movements. The manner in which vision mediates these processes, however, has yet to be clarified. The primary 
objective of the present work was to examine the modificational role of vision during goal-directed limb movements. In addition, a current model for the control of rapid aimed limb movements, recently proposed by Meyer, Abrams, Kornblum, Wright, and Smith (1988) was evaluated. My interest was to examine selected predictions of the model and the role of vision within the framework of the model.

The Stochastic Optimized Dual Submovement Model of Meyer et al. (1988) is the latest in a line of submovement models for limb control. Before describing the model, a brief survey of the relevant literature provides a foundation for my discussion.

\section{Woodworth (1899): A Beginning}

One of the earliest investigations into the control of goal-directed arm movements was conducted by R.S. Woodworth in 1899. His interest in how movement speed influenced movement accuracy led to his pioneering work on the nature of the speed-accuracy tradeoff and visual control in rapid arm movements. Woodworth's (1899) observation that movement accuracy decreased as movement speed increased stimulated his search for the sources of this speed-accuracy tradeoff.

Woodworth's (1899) experimental paradigm was simple by today's standards, and yet it established the basic foundation of the paradigms used today. The subjects' task 
was to repeatedly draw lines of a specified distance on chart paper which was continually displaced by a kymograph rotating along its horizontal axis The surface of the paper was visible through a slot on a table top, oriented perpendicularly to the direction of paper displacement The subjects' movements were paced by a metronome, such that a complete movement back and forth was made with each beat of the metronome Woodworth used a range of 20 to 200 beats per minute to pace the movements In addition, subjects had to perform under two visual conditions In the eyes open condition, subjects had full visual feedback about their movements In the eyes closed condition, subjects kept their eyes closed during the task, thus receiving no visual feedback regarding their movements

Woodworth (1899 proposed that rapid aimed movements consisted of two phases: an initial adjustment phase and a current control phase According to woodworth, the initial adjustment phase corresponded to the transport of the hand toward the target. This early phase was supposedly preprogrammed and ballistic The latter, current control, phase was an error correction phase, allowing for precision in the movement Woodworth 1899) posited that visual feedback was an important source of sensory information upon which adjustments in the current control phase were based. Thus, woodworth was interested not only in the effect of 
movement speed on accuracy, but also on the role and importance of visual feedback during the movement. He expected that unless movements were of sufficient duration to allow enough time to process visual feedback, there would be no benefit from vision during the movement. Woodworth's experiments provided the first attempt to determine the speed at which visual information can be processed.

The results of Woodworth (see Figure 1, eyes open condition) showed that as movement speed increased, movement accuracy deteriorated, reaching a plateau at movement rates above 140 cycles per minute. 1 As movement speed increased, accuracy decreased. Also illustrated in Figure 1 are the results from the eyes closed condition. In this condition, errors were generally greater compared to the eyes open condition, and remained relatively constant across the different movement speeds. What is evident in woodworth's results is that at higher movement rates (at $140 \mathrm{c} / \mathrm{min}$ and above) the accuracy of movements made with the eyes open was no greater than those made with the eyes closed. As the average duration of movements increased, greater benefits were accrued from having vision available.

In his discussion, woodworth suggested that movements made with full visual feedback were composed of both the

1 A similar result was obtained in a later experiment in which woodworth varied movement speed by instructing subjects to either draw slowly or quickly. 


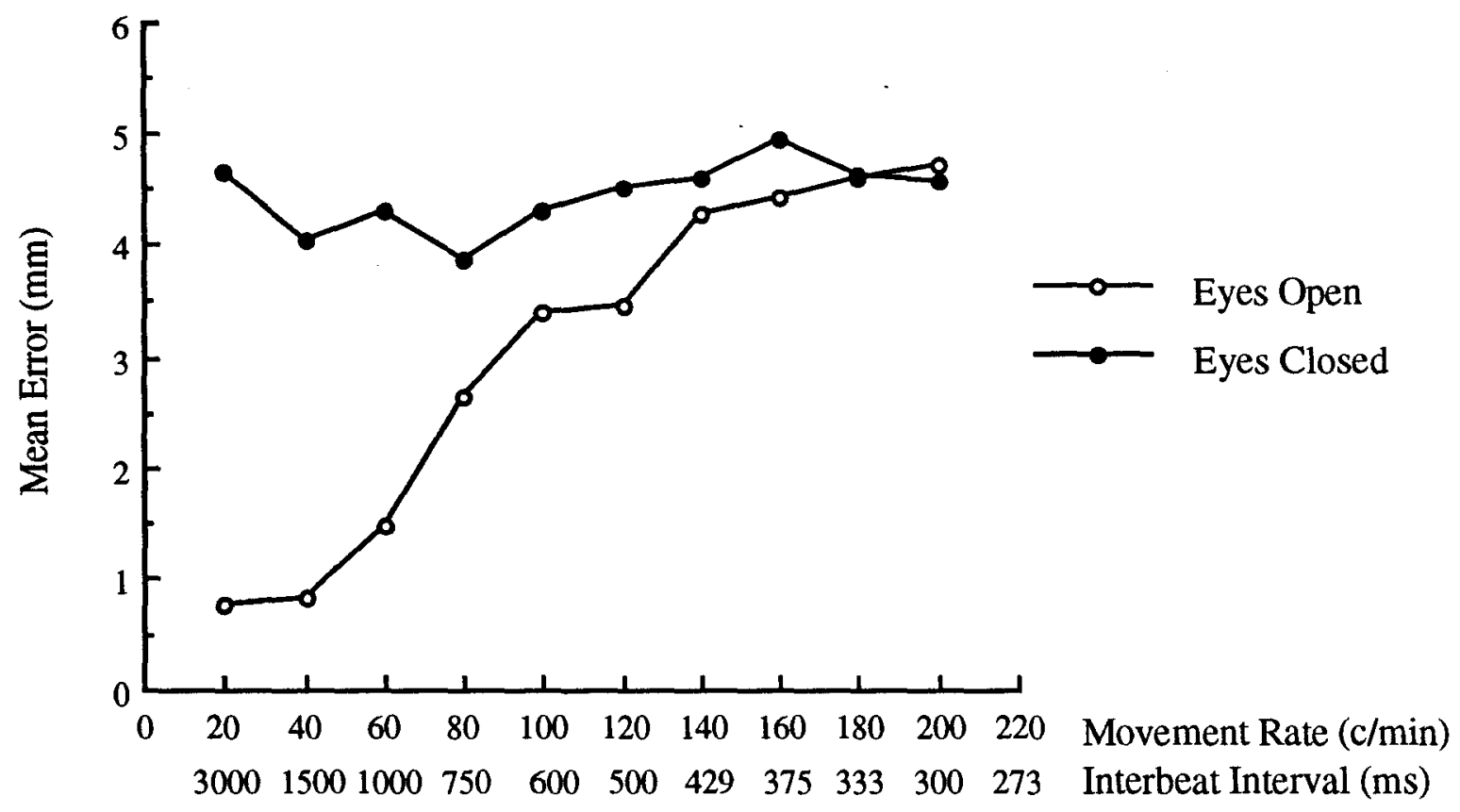

Eigure 1. Results from Woodworth's (1899) experiments. The data presented are from four subjects (Tables I - IV, Woodworth, 1899). The interbeat interval has been calculated from the corresponding movement rate and shown on the same $\mathrm{x}-$ axis. 
initial adjustment and current control phases, whereas under conditions of no vision, movements only consisted of the initial adjustment phase. He concluded that increased movement speed did not affect the initial adjustment phase since movement errors remained relatively constant in the eyes closed condition. Instead, he inferred that the negative influence of movement speed was in preventing error corrections mediated by visual feedback in the current control phase. He postulated that "the bad effect of speed consists in rendering impossible a delicate current control in preventing those later and finer adjustments by means of which a movement is enabled to approximate more and more closely to its goal" (Woodworth, 1899, p. 42). In addition to these conclusions, Woodworth proposed that the time to process visual feedback from the movement was approximately $450 \mathrm{~ms}$, since at higher movement rates, there were no differences in accuracy between the full vision and no vision conditions. Present estimates of the time of visual feedback processing are considerably shorter than that proposed by Woodworth (see Carlton, 1992, for a review).

\section{Fitts Law}

It was not until the work of Fitts (1954; Fitts \& Peterson, 1964) that a formal description of the relation between movement speed, amplitude and accuracy requirements emerged. In his now classic reciprocal tapping task (Fitts, 
1954), Fitts had subjects make reciprocal tapping movements with a stylus between two metal target plates. Subjects were instructed to make their reciprocal movements as rapidly as possible, with the constraint that they hit the targets on at least $95 \%$ of the taps. Fitts manipulated the amplitude of the movement and the widths of the targets. He found that the relation between movement duration, movement amplitude and target size could be described by a logarithmic equation describing a linear relation between movement duration and the logarithm of the ratio of twice the movement amplitude divided by the target width. The relation is expressed by Equation 1:

$$
M T=a+b \log _{2}(2 D / W)
$$

In Equation 1, MT is the average movement time to move from one target to the other; $\mathrm{D}$ is the movement amplitude or distance between the targets; $W$ is the width of the target, and $\mathrm{a}$ and $\mathrm{b}$ are empirically derived constants. The term $\log _{2}(2 \mathrm{D} / \mathrm{W})$ has been defined as the index of difficulty of the movement (Fitts, 1954; Fitts \& Peterson, 1964).

The logarithmic speed-accuracy relation observed by Fitts (1954) has been found to be highly reliable and to hold under various conditions (see Keele, 1981; Meyer et al., 1990, for reviews). This relation has subsequently been termed Fitts' Law (Crossman \& Goodeve, 1963/1983; Keele, 1968, 1981). Fitts (1954; Fitts \& Peterson, 1964) chose to 
interpret his results in terms of information theory. He viewed the human motor system as a noisy, 1imited capacity information channel (Fitts, 1954; Fitts \& Peterson, 1964). Other investigators (e.g., Crossman \& Goodeve, 1963/1983), however, have questioned this approach and have proposed alternative explanations for Fitts' Law.

\section{Iterative corrections Model}

In his interpretation, Fitts (1954) did not consider a feedback based explanation for the speed-accuracy relation he formalized. Subsequent investigators proposed that Fitts' Law could be derived from a feedback based model. The iterative corrections model, originally proposed by crossman and Goodeve (1963/1983) and later elaborated by Keele (1968), was proposed as an alternative to information theory for explaining Fitts' Law.

The iterative corrections model proposes that a movement toward a target is composed of a series of discrete submovements. The model makes some assumptions with regards to these submovements. Each submovement is assumed to be of constant duration, this duration being equivalent to the minimal time required to process feedback (Crossman \& Goodeve, 1963/1983; Keele, 1968). Thus, if there are $n$ submovements, and each submovement takes a certain constant amount of time, $t$, to complete, the total movement duration would simply be equal to nt. A second assumption is that 
each submovement moves a constant proportion of the remaining distance to the target. A third assumption is that submovements are guided by sensory feedback, of which visual feedback was considered to be the most important (Keele, 1968, 1981). Once a movement to a target has been initiated, the submovement sequence is supposed to continue until the target is reached or the remaining distance is less than half the target width. Keele (1968) has mathematically derived Fitts' Law using this model.

A number of problems with the iterative corrections model have been identified by other authors (e.g., see Keele, 1981; Meyer, Smith, \& Wright, 1982; Meyer et al., 1988, 1990; Schmidt, Zelaznik, \& Frank, 1978). A model which incorporates discrete error corrections must find justification for such a process in the dynamics of a movement. Specifically, it would be necessary to find discontinuities in the movement dynamics, suggesting the presence of discrete amendments to the movement. Although such evidence is present (Annett, Golby, \& Kay, 1958; Crossman \& Goodeve, 1963/1983; Jagacinski, Repperger, Moran, Ward, \& Glass, 1980; Langolf, Chaffin, \& Foulke, 1976), movements may seldom contain more than one or two submovements, if any at all (e.g., Carlton, 1979, 1981; Elliott, Carson, Goodman, \& Chua, 1991; Langolf et al., 1976; Mackenzie, Marteniuk, Dugas, Liske, \& Eickmeier, 1987). The 
iterative corrections model is unable to deal with movements in which no corrections are observed. The model faces further problems with findings which show that submovement durations are not necessarily equal in duration (e.g.. Jagacinski et al., 1980; Langolf et al., 1976), and that initial submovement velocity and amplitude may vary depending on target distance and width (e.g., Jagacinski et al., 1980; Langolf et al., 1976; Meyer et al., 1988). These findings violate the assumptions and predictions of the model.

As an alternative to a multiple correction model (Crossman \& Goodeve, 1963/1983; Keele, 1968) a "terminal correction" model of aiming has been proposed (Beggs \& Howarth, 1988; Howarth \& Beggs, 1985; Howarth, Beggs, \& Bowden, 1971). The model proposed by these authors has been referred to as a single correction model by other authors (e.g., Elliott et al., 1991; Keele, 1981). However, it has subsequently been clarified that the model does not constrain movements to a single correction (Beggs \& Howarth, 1988; Howarth \& Beggs, 1985). Rather, the emphasis of the model is on where in space, relative to the target, the final correction to a movement occurs (Beggs \& Howarth, 1988; Howarth \& Beggs, 1985). I have therefore chosen to refer to the model as a "terminal correction" model.

The basic premise of the terminal correction model is that movement accuracy is related to the distance the hand is 
from the target at the time the final amendment to the movement occurs. Greater accuracy is achieved when the hand is closer to the target at the time of correction. It is assumed that for movements requiring greater accuracy, the velocity of the limb is decreased, bringing the hand closer to the target prior to the final correction Like the iterative corrections model, the correction is mediated by visual feedback Unlike the iterative corrections model, greater precision is achieved not by making a greater number of visually based corrections, but by decreasing the speed of the movement and increasing the proximity of the limb with respect to the target prior to the final correction The Stochastic Optimized Dual submovement Model Accounts of the speed-accuracy tradeoff have not been limited to feedback-based models of limb control In reaction to multiple correction models of limb control (e $\mathrm{g}$, Crossman \& Goodeve, 1963/1983, theories of impulse variability have been advanced as an alternative account of the relation between movement time, amplitude, and accuracy (e g., Schmidt et al, 1978 The basic premise of the impulse variability model is that neuromotor noise present in the motor system causes variability in the output of the system Due to this noise, movements of greater velocity are associated with greater variability The impulse variability model has stimulated a considerable amount of research on 
force variability and speed-accuracy principles This literature is beyond the scope of the present review and readers are referred to the relevant literature (e g, Meyer et al , 1982; Schmidt et al , 1978; Schmidt, Zelaznik, Hawkins, Frank, \& Quinn, 1979; Schmidt, Sherwood, Zelaznik, \& Leikind, 1985; Sherwood, 1986; see also Newell, 1980; Hancock \& Newell, 1985, for a discussion of the space-time approach One result which has emerged from the interest in impulse variability (e g, Meyer et al, 1982; Schmidt et al., 1979) is the latest submovement model for limb control The dual submovement model of Meyer et al 1988 has been proposed to account for the speed-accuracy tradeoff in spatially constrained tasks The model makes some mathematical and empirical predictions with regards to the speed-accuracy tradeoff and rapid aimed movements The empirical predictions are of concern here

The optimized dual submovement model proposes that rapid aimed movements are made up of a primary and secondary submovement whose durations are optimized in order to cope with a noisy motor system The dual submovement model makes six basic assumptions.

The first assumption involves the notion of noise present in the neuromotor system This concept has been proposed earlier (Meyer et al , 1982; Schmidt et al, 1978, 1979) It is this neuromotor noise which is proposed to lead 
to variability in the output of the motor system Due to this noise, a person making rapid aimed movements cannot always produce the exact same movement across trials The second assumption relates to the number of submovements produced to reach the target region In contrast to the multiple correction model crossman \& Goodeve, 1963/1983; Keele, 1968 , the dual submovement model assumes that rapid goal-directed movements are composed of either one or two component submovements, this number being independent of the movement amplitude and target width The initial, or primary, submovement is assumed to be programmed to end at the center of the target A secondary submovement, prepared through sensory feedback (e g, visual) is assumed to occur if the primary submovement misses the target due to motor output variability otherwise, if the primary submovement hits the target, then no secondary submovement occurs

A third assumption involves the distribution of the submovement endpoints The model assumes that due to neuromotor noise, the endpoints of the primary and secondary submovements are normally (or symmetrically) distributed about the center of the target

A fourth assumption is that the standard deviations of the primary and secondary submovement endpoints are proportional to the average velocity of the respective 
submovements (see also Meyer et al., 1982; Schmidt et al., 1978, 1979). This would require that there be a strong correlation between the average velocity of the submovement and the magnitude of the neuromotor noise associated with the production of that submovement (Meyer et al., 1988). In a spatially constrained movement task, the standard deviation of the secondary submovement endpoints is constrained by the requirement that the movement end within the target region on at least $95 \%$ of the trials.

In the model, the total movement time is simply the sum of the durations of the primary and secondary submovements. In a spatially constrained task in which the objective is to hit the target as quickly and as accurately as possible, the model claims that movement times are minimized conditional on the submovement endpoint variability and neuromotor noise. This optimization of the total movement time is achieved through an ideal compromise between the durations of the component submovements (Meyer et al., 1988).

The last assumption incorporated by the model deals with the processing of information for movement production. This assumption will be discussed later as it is an issue which concerns the discussion on the role of vision within the framework of the model. 
The dual submovement model makes a number of quantitative predictions concerning the average total movement time, the standard deviations of submovement endpoints, average durations of the component submovements, and the relative frequencies of secondary submovements and errors, on how they relate to target distance and width (see Meyer et al., 1988 for details). Meyer et al. (1988) have provided some quantitative and qualitative support for these predictions in a wrist rotation task in which rotation of a hand held handle displaces a cursor on a monitor toward a target region. Although Meyer et al. (1988) have provided some support for their dual submovement model, there are some issues concerning the role of vision on which their model is not very clear or consistent. This one aspect of their model provides one of the focal points of this investigation.

Visual-Motor Control and the optimized Dual Submovement Model The optimized Dual Submovement Model

The dual submovement model claims that rapid aimed movements are composed of either a primary submovement or a primary and secondary submovement. Meyer et al.'s (1988) discussion on the manner in which these component submovements are prepared raises some questions about the role of vision as it relates to the model. 
In their model, Meyer et al. (1988) assume that the preparation of the primary submovement requires the processing of information pertaining to movement amplitude or target distance, and the width of the target. In addition, this submovement is programmed to hit the center of the target. If the initial submovement is planned prior to initiation, then perhaps elimination of visual feedback may not have a significant impact upon the characteristics of this submovement. Indeed, the results of the invisiblecursor condition of Experiment 2 in Meyer et al. (1988) show no apparent effects on the nature of the primary submovement when visual feedback was eliminated at the onset of the movement. In this experiment visual feedback availability was manipulated in order to examine its effects on performance in a wrist rotation aiming task. In the invisible-cursor condition visual feedback was eliminated by extinguishing the cursor which the subject moved to the target. The target remained illuminated throughout the movement. In the visible-cursor condition subjects had vision of both the cursor and the target at all times. In both visual conditions, there was no vision of the hand; thus visual feedback was with respect to the cursor only. From Meyer et al.'s (1988) assumptions and results, it would be plausible to expect that eliminating vision of the target rather than the cursor at movement initiation would also have 
no impact upon the primary submovement. Whether this would be true in a situation in which visual feedback was eliminated completely (eliminate vision of both cursor and target) remains to be investigated.

of even greater interest with respect to Meyer et al.'s (1988) model are the questions raised by their assumptions about the preparation of the secondary submovement. With respect to the secondary submovement, Meyer et al. (1988) assume:

A secondary submovement may be prepared on the basis of the target's location and visual or kinesthetic feedback regarding dynamic characteristics (e.g., velocity and acceleration) of the primary submovement. The process that prepares secondary submovements may also use "feedforward" (efference copy) from the primary submovements (von Holst, 1954). We assume that feedback and feedforward are processed "on the fly" during movement production, so that essentially no delay ensues between the end of a primary submovement and the beginning of a subsequent secondary submovement. (...) The model does not allow for "dead time" (i.e.. intermediate pauses during which movement velocity is zero over an extended time interval) after a primary submovement has begun. (p. 347)

Although Meyer et al. (1988) propose and initially

acknowledge that preparation of the secondary submovement may use feedforward information, the authors seem to opt for a feedback (visual) based preparation process. Moreover, although they contend that this feedback may be feedback concerning the dynamic characteristics of the primary submovement and that processing occurs "on the fly" during movement production, they later adopt an explanation (to 
explain results in the invisible-cursor condition, Experiment 2) that is not consistent with their assumption of a zerodelay between the end of the primary submovement and the start of the secondary submovement.

If the model does not allow for any delay between submovements, then this would necessitate the preparation of the secondary submovement based on feedforward information or on feedback information from the earlier portions of the primary submovement. This constraint further implies that preparation of the secondary submovement cannot be made on the basis of visual feedback of the primary submovement endpoint (Carson, Goodman, Chua, \& Elliott, in press). Processing of such feedback would take time and would therefore not be in agreement with the model's zero-delay between submovements. Therefore, if visual feedback from the primary submovement is used to prepare the secondary submovement, it must be feedback from the earlier stages of this initial submovement, providing it is of sufficient duration. It would be unlikely that feedback from the late stages of the primary submovement, especially its endpoint, can be used. Problems related to this issue are evident in Meyer et al.'s (1988) discussion concerning the invisiblecursor condition in their second experiment.

In the invisible-cursor condition (Meyer et al., 1988, Experiment 2), the authors found that the error rates 
(target region missed completely) increased, as would be expected. Additionally, the frequency of secondary submovements did not differ from that found in a condition in which the cursor was visible. This latter finding suggests that the presence of secondary corrections are not dependent on visual feedback (see Jeannerod, 1988 for a discussion), and is consistent with the findings of others (e.g., Carson, Goodman, \& Elliott, 1992; Elliott et al., 1991; Jeannerod, 1984).

Meyer et al.'s (1988) explanation for the increased error rates is predicated on the assumption that accurate secondary submovements require visual feedback of the primary submovement outcome. The authors' explanation is as follows:

Our account of error rates under the invisible-cursor condition assumes that when subjects cannot see the cursor, they are uncertain about the spatial positions of primary submovement endpoints. According to this view, subjects make guesses about where their inaccurate primary submovements have ended, and on the basis of these guesses, they produce attempted corrective secondary submovements as if the cursor were visible. (p. 363)

That their explanation involves feedback concerning the primary submovement endpoint poses a problem for their model, in light of the discussion earlier regarding the model's zero-delay constraint.

If the preparation of an accurate secondary submovement is dependent upon visual feedback from the primary submovement, then this raises the question of what 
visual information pertaining to the initial submovement would be important? It has been argued that this visual information cannot be concerned with the endpoint of the primary submovement If such were the case, then some delay must exist between the end of the primary submovement and the beginning of the secondary submovement Therefore, it would imply that visual information regarding the earlier segments of the primary submovement would be important for movement accuracy (cf. Beaubaton \& Hay, 1986; Carlton, 1981) The contribution of Vision During the Early and Latter stages of Aiming Movements

The implication that vision is important primarily in the latter stages of a reaching movement stems from models which suggest that amendments are mediated primarily by a comparison of the position of the hand with the position of the target From the perspective of these models (e g, Carlton, 1979, 1981; Crossman \& Goodeve, 1963/1983; Howarth et al., 1971; Keele, 1968; Woodworth, 1899) the initial movement of the limb toward a target is ballistic and visually based error corrections do not occur until the latter portion of the overall movement e g, Paillard, 1980) This view has led to suggestions that visual information from the early stages of an aiming movement is not of any benefit in terms of movement accuracy 
Carlton (1981) has questioned the effectiveness of early visual feedback in improving pointing accuracy. In his study, Carlton (1981) manipulated the amount of visual information a subject received during an aiming movement. By using a shield, he eliminated from the subject's view the initial 25, 50, 75, and 93 percent of the movement trajectory to the target. He found that error rates increased only when more than 50 percent of the movement was hidden from view, leading him to conclude that visual information from the initial portions of the movement had little influence on its accuracy .

Carlton's (1981) conclusion was based upon the similarity of errors between the full visual feedback condition and the conditions in which the initial 25 and 50 percent of the movement was masked. Although movement times in these conditions were also similar, this does not exclude the possibility that a greater proportion of the movement may have been spent in the latter portion of the movement when vision became available, allowing sufficient time to amend the movement if necessary and achieve the required accuracy. Moreover, Carlton's (1981) manipulation did not include any conditions in which vision was available only on the early stages of the movement. Therefore, he was not able to determine directly whether early visual information would 
increase accuracy relative to a condition in which no visual feedback was provided.

Another experiment in which visual feedback was selectively excluded during pointing movements was conducted by Beaubaton and Hay (1986). Unlike Carlton (1981), these authors also included a condition in which vision was available only in the first half of the trajectory. Subjects performed under five feedback conditions. These were: complete feedback (full vision), no feedback (no vision with no endpoint KR), error feedback (no vision with endpoint KR), initial feedback (vision on first half of trajectory), and terminal feedback (vision on the second half of the trajectory). Subjects also performed at four specified movement durations: $110-150 \mathrm{~ms}, 150-190 \mathrm{~ms}, 190-230 \mathrm{~ms}$, and 230-270 ms. Examination of radial error revealed that subjects performed most accurately in the complete feedback and terminal feedback conditions, and worst on the initial feedback and no feedback conditions, with the error feedback condition being intermediate. 2 These findings led the authors to conclude that the early visual information during the pointing movement did not benefit performance (Beaubaton \& Hay, 1986). Some caution may be warranted in interpreting these results, however. Since it was also the authors.

2 Beaubaton and Hay (1986) labelled radial error as constant spatial error. 
purpose to investigate the contribution of visual information to feedforward processes in the control of aiming movements, the five visual feedback conditions were not equated in terms of the knowledge of results (KR) relating to movement accuracy. Subjects did not receive KR during the no feedback and initial feedback conditions. It is possible that inequality of $\mathrm{KR}$ across conditions may have confounded the results, particularly if the feedback conditions were performed in blocked fashion.

In contrast to the studies of carlton (1981) and Beaubaton and Hay (1986), earlier work by Conti and Beaubaton (1976) demonstrated a positive contribution of early visual information to movement accuracy. In this study (Conti \& Beaubaton, 1976), six vision conditions were employed: full vision, no vision, vision on the first half of the trajectory, vision on the second half of the trajectory, vision on the middle third of the trajectory, and vision during the first and final thirds of the trajectory. Subjects were instructed to make either "fast" (<200 ms), "normal" (200-700 ms), or "slow" (>700 ms) movements. In the no vision condition, movements were equally inaccurate for all three classes of movements. When movements were executed at a "normal" or "slow" speed, movements were most accurate when either full vision or vision of the latter part of the trajectory was available. of interest is that when 
visual feedback was limited to the first half of the movement trajectory, accuracy improved over that when no visual feedback was provided. This suggests that early visual cues could have been used later in the movement to improve its accuracy (Jeannerod, 1988; Paillard, 1982).

More recently, additional evidence to suggest the benefits of early visual information has been provided by Young and Zelaznik (1992). The subjects' task was to make rapid aiming movements to either stationary or moving targets in movement time ranges of $150 \mathrm{~ms}$ and $350 \mathrm{~ms}$. Of interest was the absence of accuracy differences for movements made under "lights-on" and "lights-off" conditions. Also of importance was the nature of the "lights-off" condition. In this condition, visual feedback was eliminated by extinguishing ambient lighting $50 \mathrm{~ms}$ after the initiation of the movement. Thus, in both vision conditions, visual information was available for the first $50 \mathrm{~ms}$ of the movement. Given that removing visual information $50 \mathrm{~ms}$ after the movement had been initiated did not reduce accuracy with respect to the "lights-on" condition, this suggests that subjects were able to obtain useful information from the early portion of the movement (Young \& Zelaznik, 1992).

It seems reasonable to believe that for relatively rapid aiming movements, early visual information could significantly contribute to accuracy. If visual feedback is 
to be utilized, the intrinsic delay in visuomotor processing implies that visual information must be picked up relatively early in the movement and used to extrapolate the final position of the hand (Jeannerod \& Prablanc, 1983). The importance of early visual information has already been discussed with respect to the dual submovement model proposed by Meyer et al. (1988). I have argued that preparation of the corrective submovement cannot be mediated by visual feedback of the endpoint of the initial submovement. In light of the remarks of Jeannerod and Prablanc (1983), it may be that secondary submovements are prepared on the basis of the projected submovement endpoint, the calculation of which may be based upon early visual information from the movement trajectory. Note that Meyer et al. (1988) did initially acknowledge that corrective submovements may be prepared on the basis of feedforward information.

The Modificational Role of Vision and the Nature of

\section{Visual-Motor Regulation}

Submovement models of limb control have generally emphasized the importance of vision for discrete error corrections (e.g., Carlton, 1979; Crossman \& Goodeve, 1963/1983; Keele, 1968; Howarth et al., 1971). Support for submovement models have come from the observation that discrete adjustments to movements do occur (e.g., Carlton, 1979; Elliott et al., 1991; Jagacinski et al., 1980; 
Jeannerod, 1984; Langolf et al., 1976). However, the role that vision plays in the process remains to be elucidated (Elliott et al., 1991). If discrete adjustments are based solely on visual information, then these models (e.g., Crossman \& Goodeve, 1963/1983; Keele, 1968; Howarth et al., 1971) would predict that corrections should be evident in conditions of full visual feedback, in which vision can mediate the comparison of the hand and target position (see Elliott, 1988, for discussion of the relative importance of limb and target information). In the absence of visual feedback, the models would predict that adjustments should not be evident (Elliott et al., 1991), implying that movements should go uncorrected. Findings which demonstrate the presence of discrete adjustments in the absence of visual feedback (e.g., Abrams, Meyer, \& Kornblum, 1990; Carson et al., 1992; Elliott et al., 1991; Jeannerod, 1984; Meyer et al., 1988) therefore pose a problem for these submovement models. An additional problem is the demonstration that pointing movements to visual targets can be modified despite the absence of visual feedback of the limb (e.g., Pélisson, Prablanc, Goodale, \& Jeannerod, 1986; Prablanc, Pélisson, \& Goodale, 1986). These results tend to discredit the visual feedback hypothesis of Fitts' Law (e.g., Crossman \& Goodeve, 1963/1983; Keele, 1968) which implies that this speed- 
accuracy relation relies on visually based corrections (Jeannerod, 1988).

Meyer et al. (1988) have suggested that vision mediates the execution of more accurate corrective submovements. In their study (Meyer et al., 1988), the authors proposed that the presence of secondary submovements despite the absence of visual feedback was a consequence of the strategy adopted by subjects. They maintained that subjects prepared and executed their primary submovements in the usual manner, and supplemented them with secondary submovements even though visual feedback was absent (see Meyer et al., 1988). These secondary submovements were assumed to be based on the subject's guesses about the endpoints of the primary submovements (see above discussion). Therefore, the implication is that the presence of secondary submovements might not necessarily be mediated by the availability of visual information; rather, it may be the relative accuracy of these submovements on which vision impresses its influence.

The nature of feedback-based error corrections has historically been implied to be in the form of discrete amendments to the movement trajectory. Corrective submovements (e.g., Crossman \& Goodeve, 1963/1983; Keele, 1968; Meyer et al., 1988) have been defined as impulses "responding to and reducing visual error" (Keele, 1981, 
p.1393). These discrete amendments have been inferred from discontinuities evident in the kinematic profiles of aiming movements. Moreover, these discontinuities have primarily been in the form of discrete, secondary adjustments in the velocity or acceleration profiles (e.g., Brooks, 1974;

Crossman \& Goodeve, 1963/1983; Elliott et al., 1991;

Jagacinski et al., 1980; Langolf et al., 1976). One measure in particular which has been used to infer the presence of trajectory modifications has been "zero crossings", or negative to positive transitions in the acceleration profile (e.g., Brooks, 1974; Carson et al., 1992, in press; Elliott et al., 1991), for which "motor commands for successive steps are supposed to be given only after intermittent referral to peripheral and central information" (Brooks, 1974, p. 306). Although the use of zero crossings may provide an indicator of the presence of what may be termed as discrete "submovements", one obvious limitation is that this may not be the only form of modification which could be made to a limb movement.

Recent discussions (e.g., Carson et al., 1992, in press; Elliott et al., 1991; Pélisson et al., 1986) on the nature of movement regulation have given rise to the idea that visuomotor control of pointing may also proceed in a "continuous" or "pseudo-continuous" fashion. The emergence of this concept has been stimulated by findings which have 
shown that the presence of visual information contributes to performance accuracy (Carson et al., 1992; Elliott et al., 1991) despite the absence of measurable additional processing time (Carson et al., 1992; Pélisson et al., 1986) and of additional discrete corrections to the trajectory (Carson et al., 1992; Elliott et al., 1991; Pélisson et al., 1986). Moreover, additional impetus for this perspective has come from the demonstration that, whereas the availability of visual information is a potent determinant of movement accuracy, the presence of discrete corrections itself has little influence on accuracy (Carson et al., in press; Elliott et al., 1991). The term "continuous" control has been offered to reflect a regulatory process whereby vision might subserve graded, as opposed to discrete, adjustments to the impulses acting upon the limb (Elliott et al., 1991). "Pseudo-continuous" control has been suggested to reflect the "impossibility in practice to differentiate between continuous control and early discrete correction filtered by the inertial properties of the moving limb" (Pélisson et al., 1986, p.309). Because the nature of the control processes must be inferred indirectly from observation of the active limb, it is possible that the response of the muscles, and therefore the limb, may not entirely reflect the nature of the underlying neural impulses. 
In light of the possibility that visual control of pointing movements may proceed in a continuous, as well as a discrete fashion, it may not be too surprising therefore, that previous investigators (Carson et al., 1992; Elliott et al., 1991) have failed to reveal any differences in the number of trajectory adjustments between different visual conditions (cf. Carson et al., in press). These authors (Carson et al., 1992; Elliott et al., 1991) only examined zero crossings, or secondary accelerations. Perhaps, if zero crossings are more reflective of a "discrete" mode of control, then this measure would be insensitive to "continuous" types of modifications which would therefore be overlooked. Hence, one question to consider is whether there are modifications reflective of a continuous mode of control which, in turn, can be inferred from kinematic profiles. Similarly, what kinematic indices, if any, might be sensitive to "continuous" forms of adjustments? To date, little consideration has been given to this issue.

In a recent study, Carson et al. (in press) have examined, in addition to zero crossings, the presence of "significant deviations" in the acceleration profiles (see also van Donkelaar \& Franks, 1991) of aiming movements. Significant deviations reflect uncharacteristic changes in acceleration which do not lead to a transition in the sign of the acceleration (e.g., see Carlton, 1979, 1981; Carson et 
al., in press; Meyer et al., 1988; van Donkelaar \& Franks, 1991). The addition of this measure, therefore, provides a method for detecting movement modifications to which zero crossings would be insensitive. Given that continuous visuomotor control may operate via graded variations of the "gain" of muscle activity in the active limb (Carson et al., in press; Elliott, 1992; Elliott et al., 1991), perhaps a significant deviation in the acceleration trace might serve as a kinematic index more reflective of continuous or pseudocontinuous regulation. Although viewing "significant deviations" in this manner is speculative, and may be at variance with others (cf. Carlton, 1979, 1981; Carson et al., in press; Meyer et al., 1988), perhaps it would serve as an initial step in the consideration of differing modes of control (e.g., discrete vs continuous) and their relation to observable kinematic variables. In the majority of the work to date, the presence of what may be termed as a significant deviation has been used simply to identify the end of the initial submovement (e.g., Abrams et al., 1990; Carlton, 1979; 1981; Meyer et al., 1988; Worringham, 1991), and few attempts have been made in distinguishing between different types of modifications (cf. Carson et al., in press; van Donkelaar \& Franks, 1991).

The present studies were designed to elaborate upon the role of vision in the regulation of an ongoing limb 
movement and to address some issues pertaining to the nature of this regulation. An additional purpose of this work was to examine certain predictions of the dual submovement model (Meyer et al., 1988), with the general emphasis being placed upon vision and motor control. Of interest was the influence of varying visual conditions on manual aiming performance and on the kinematics underlying these actions.

Examination of the Role of Vision in Manual Aiming and of the Stochastic optimized Dual Submovement Model

In evaluating their model, Meyer et al. (1988)

employed an aiming movement requiring wrist rotations. The rationale which they provided for using such a task was based on the demonstration that wrist rotations have been shown to exhibit different forms of the speed-accuracy tradeoff, depending on whether the task was spatially constrained or temporally constrained (e.g., see Crossman \& Goodeve, 1963/1983; Jagacinski et al., 1980; Wright \& Meyer, 1983).3 Furthermore, the analyses of movement kinematics were simplified because of the unidimensional nature of the movement, and, since the motion must be stopped entirely by antagonist muscle activity and friction, movement records

3 A linear tradeoff has been demonstrated for temporally constrained movements in which subjects are required to move as accurately as possible to a target within a specified movement duration. A logarithmic tradeoff has been demonstrated for spatially constrained movements in which subjects must terminate their movements within a specified target region as quickly as possible. 
were not contaminated by impact with a target as in stylus tapping (Meyer et al., 1988).

Although the wrist rotation task has been demonstrated to follow typical speed-accuracy tradeoff principles (Crossman \& Goodeve, 1963/1983; Jagacinski et al., 1980; Meyer et al., 1988; Wright \& Meyer, 1983), it is not very representative of arm movements in which the limb is actually transported toward some goal. The aiming task utilized in the present experiments represented a compromise between wrist rotation (e.g., Meyer et al., 1988; Wright \& Meyer, 1983) and more traditional target aiming (e.g., Carlton, 1981; Carson et al., 1990; Elliott et al., 1991; Mackenzie et al., 1987; Prablanc et al., 1986; Schmidt et al., 1979; Todor \& Smiley, 1985). The task utilized a digitizing tablet and mouse interfaced with a microcomputer. Movement of the mouse along the surface of the tablet displaced a cursor on the computer monitor such that movement of the cursor paralleled that of the mouse. Therefore, the experimental set-up was similar to that of Meyer et al. (1988) in that a zero order control function was imposed, relating the displacement of the arm to the position of a cursor displayed on the monitor. However, unlike Meyer et al. (1988), the arm movement required more closely resembled a multiarticular pointing movement, although performed along a single plane. An additional similarity to wrist rotation 
was the absence of target impact and the requirement that movements be terminated by muscle activity and friction.

\section{Experiment 1}

In this study and Experiment 2, three further issues were addressed. These concerned the distribution of submovement endpoints, the influence of visual condition on movement strategies, and the symmetry of movement dynamics. As mentioned earlier, Meyer et al. (1988) assumed that the endpoints of submovements have a normal (or symmetrical) distribution about the center of the target. However, in their experiments, these authors did not report any data concerning movement bias. Although movements end within a target region, movement bias may still be measured with respect to the center of the target. In the dual submovement model, subjects' movements are thought to be programmed to hit the target center (Meyer et al., 1988; cf. Barret \& Glencross, 1989). There are data to suggest that the above assumptions do not necessarily hold. Several aiming studies have shown a consistent tendency by subjects to undershoot targets (e.g., Elliott \& Allard, 1985; Jeannerod, 1988; Prablanc, Echallier, Komilis, \& Jeannerod, 1979; Pélisson et al., 1986; Prablanc et al., 1986). Moreover, Elliott et al. (1991) have shown that pointing movements are more likely to undershoot the target in visually degraded conditions (e.g., see also Prablanc et al., 
1979; Prablanc, Echallier, Jeannerod, \& Komilis, 1979). Similarly, Abrams et al. (1990), employing a wrist rotation paradigm, did not find movement endpoints to be distributed about the center of the target; rather, the pervasive range effect (see Jeannerod, 1988) was demonstrated in that subjects tended to overshoot near targets and undershoot distant targets (Pepper \& Herman, 1970).

It may be that in movements requiring a reasonable degree of terminal accuracy, subjects may systematically undershoot the target with their initial movement (Jeannerod, 1988). Moreover, Worringham (1991) has proposed that initial submovement amplitude may be optimized as a function of variability (cf. Meyer et al., 1988). More specifically, the hypothesis predicts that initial submovements undershoot the target, with the degree of undershooting being a function of variability in the initial submovements (Worringham, 1991). That subjects may optimize the amplitude of their initial submovements (Worringham, 1991) in addition to movement time (Meyer et al., 1988) suggests that initial submovements may not necessarily be programmed to end at the center of the target region (cf. Meyer et al., 1988). However, in terms of the dual submovement model and multiple correction models, this would imply that rapid movements would always require secondary submovements, given that the primary submovement amplitudes would fall short of the target. 
In conditions in which visual feedback is eliminated, the dual submovement model predicts that the average total movement times or overall error rates should increase (Meyer et al., 1988). Meyer et al. (1988) suggest two possible strategies which subjects may employ in the absence of visual feedback. They adopt the latter of these strategies, which proposes that in the absence of visual feedback, subjects prepare and execute their primary submovements as usual, supplementing them with secondary submovements. This would allow the maintenance of the average total movement times at their normal minimum level. The other strategy (see Meyer et al., 1988) results in an increase in movement times. Neither of the strategies proposed, and their predicted effects on movement times, are consistent with findings that in visually degraded conditions, movement times may actually be of shorter duration (e.g., Abrams et al., 1990; Carson et al., 1990, in press; Elliott et al., 1991; Elliott \& Madalena, 1987).

The analysis of movement kinematics allows for a closer investigation of the nature of the movement dynamics. The characteristics of the trajectory profiles may better elucidate movement strategies over an analysis including only behavioural indices (e.g., MT, accuracy), and, in addition, may reflect organizational principles at some level in the central nervous system (Mackenzie et al., 1987). 
Furthermore, kinematic analyses provide a means with which to test models which assume symmetry in movement dynamics (e.g., Meyer et al., 1982). Several authors have already provided evidence against the validity of such symmetry assumptions (e.g., Heuer, 1984.; Jeannerod, 1984, 1988; Mackenzie et al., 1987; Schmidt et al., 1985; Todor \& Smiley, 1985; Zelaznik, Schmidt, \& Gielen, 1986).

Experiment 1 examined the effects of varying visual conditions and target size on manual aiming performance and movement dynamics.

\section{Method}

Subjects

Subjects were 10 right-handed (Oldfield, 1971) undergraduate and graduate students ( 4 male, 6 female; age: 17 - 27 years). All subjects had normal or corrected-tonormal vision. Apparatus

Subjects were seated facing a computer monitor (AMA VGA color monitor, model SC-431VS), and a $58.5 \mathrm{~cm} \times 44.5 \mathrm{~cm}$ graphics tablet (Summagraphics summasketch II professional MMII 1812). The monitor was located approximately $65 \mathrm{~cm}$ in front of the subject, and raised $16 \mathrm{~cm}$ from the table surface (91.5 $\mathrm{cm}$ from the floor) to bring it closer to eye level. The graphics tablet was placed directly in between the subject and the monitor, at a level which allowed the subject 
to comfortably rest the forearm horizontally on the tablet surface.

The subject held a mouse (Summasketch 4-button mouse) with the right hand and made all movements with this mouse. The mouse is fashioned such that at its anterior end is a clear plastic pointer, the center of which is demarcated by a circle 0.5 inches in diameter. Two lines/wires intersect at the center of this circular area, forming a crosshair within the circle. It is the coordinates of the crosshair intersection on the tablet surface which is translated by the graphics tablet and computer as the location of the mouse. Subjects held the mouse with the tip of their index finger placed within the crosshair circle (see Figure 2). The location of the mouse (crosshair) on the tablet, and thus the location of the index finger, was translated into the coordinates of the cursor on the monitor. This translation was performed with unity gain, such that a $1 \mathrm{~mm}$ displacement of the mouse crosshair resulted in a $1 \mathrm{~mm}$ displacement of the cursor on the monitor. The cursor appeared as a blue solid circle $3 \mathrm{~mm}$ in diameter. The output signal from the graphics tablet was sent to a computer (AMA325 386 computer), and was sampled at a rate of $138.85 \mathrm{~Hz}$.

In order to constrain movement of the mouse to a single dimension ( $y$-axis), a wooden track, fashioned out of two halves of a metre stick, was secured to the tablet 
surface along its midline (see Figure 3 The track allowed for the mouse to be moved forward and backward freely, while limiting lateral displacement to approximately $1 \mathrm{~mm}$ To reduce movement resistance due to friction, paper masking tape was used to cover the bottom surface of the mouse Since all movements were to be made without vision of the limb, a sheet of black plastic was mounted over the tablet to shield the limb from view For illustrations of the apparatus, see Figures 2 and 3

Procedure

A repeated measures $(2 \times 3$ factorial design was employed The two independent variables were vision condition (Vision-Cursor: VC; No Vision-Cursor NC and target size $(5 \mathrm{~mm}, 10 \mathrm{~mm}, 20 \mathrm{~mm})$ A single target distance of $130 \mathrm{~mm}$ was used, thereby yielding three index of difficulty levels (37,4 7, 5.7) (see Equation 1

The subject was seated facing the graphics tablet and monitor, with the midline aligned approximately with the monitor. In this orientation, the track on the tablet surface was to the right of the subject's midline, thereby allowing for movements of the right limb to be made comfortably within ipsilateral space The subject sat sufficiently close to the tablet such that the ensuing reaching movements did not necessitate full extension of the arm Ambient illumination within the black-walled experiment 

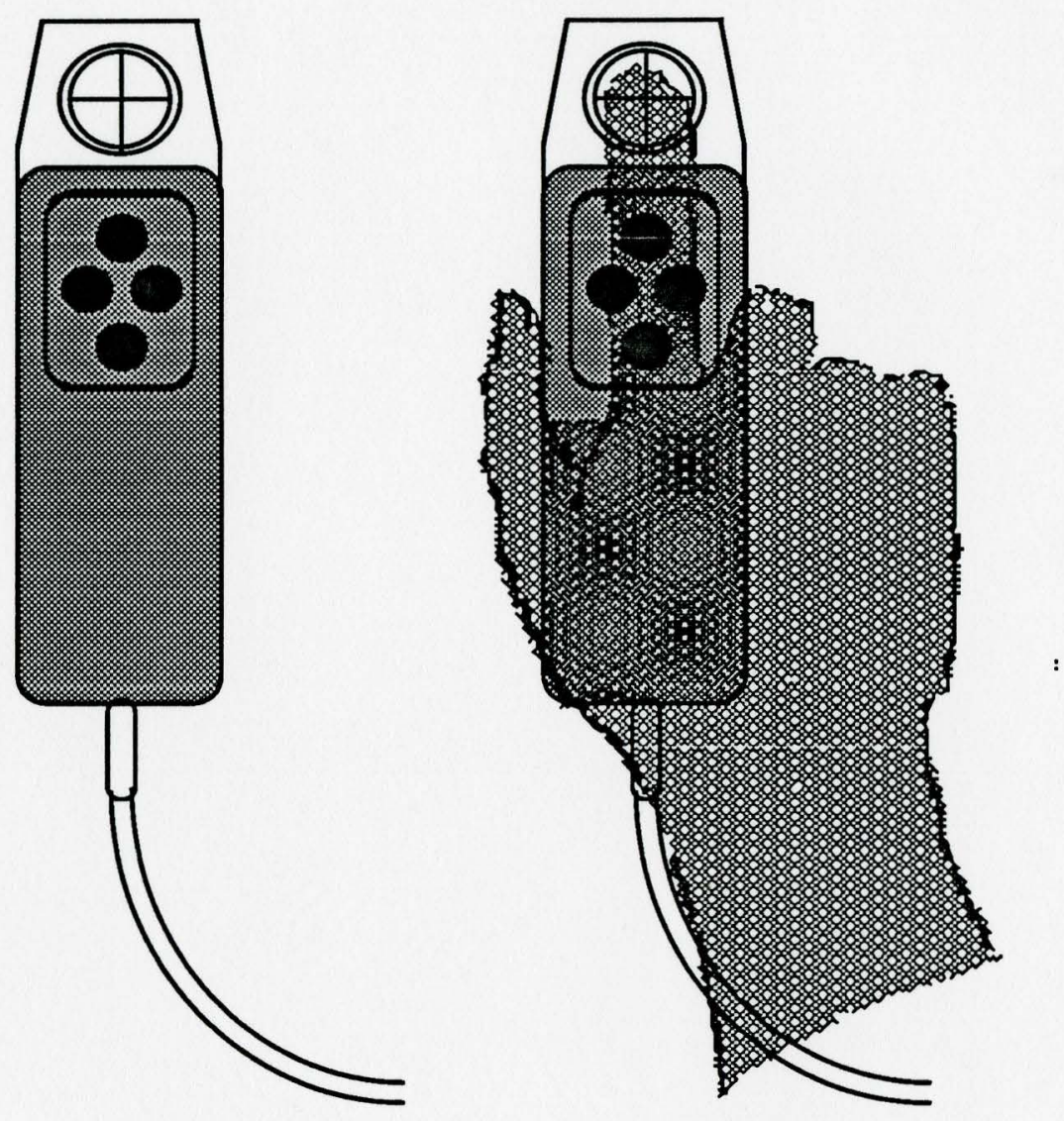

Figure 2 Diagram of graphics tablet mouse and the manner in which subjects held the mouse during the aiming movements 


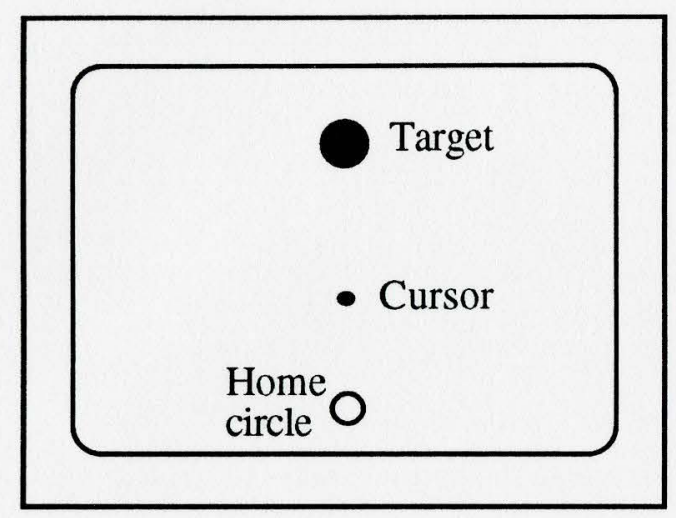

Computer Monitor

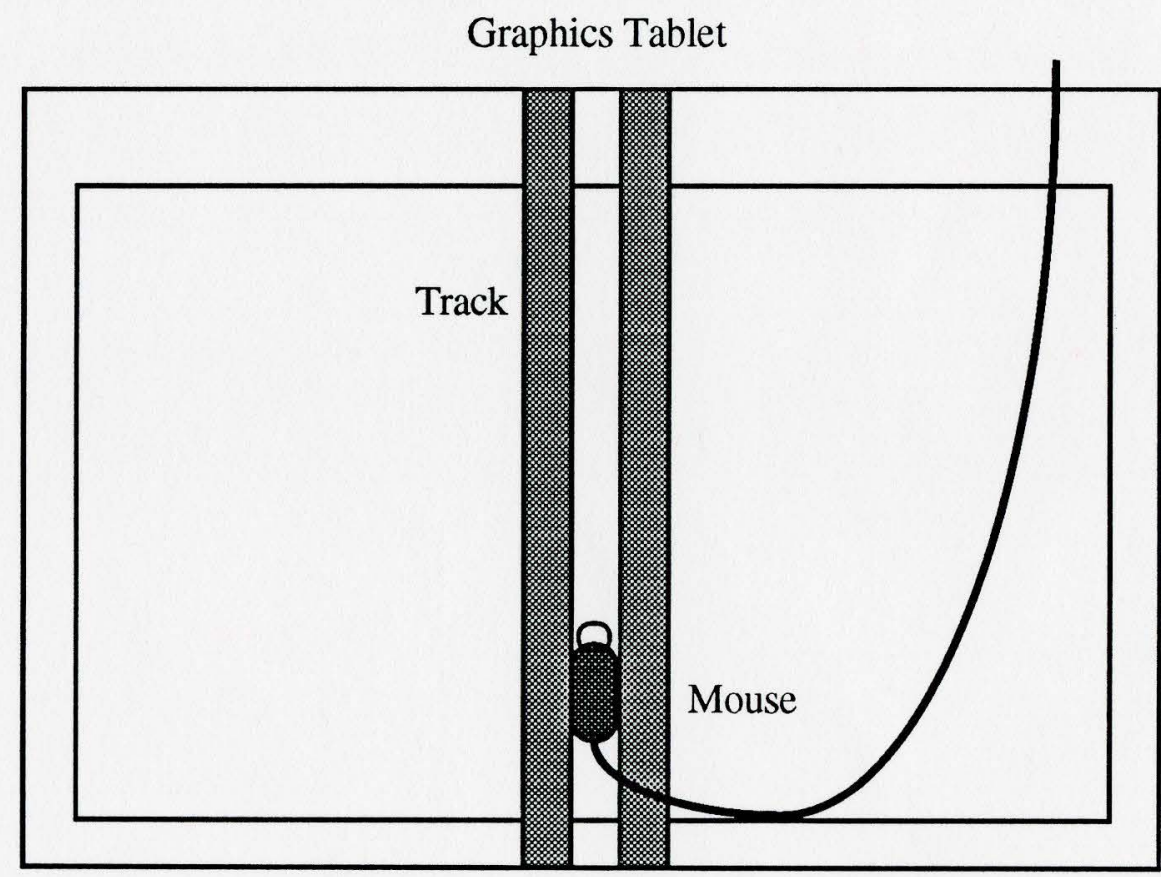

Figure 3 Diagram of graphics tablet and computer monitor 
chamber was provided by a table lamp with a 3 watt neon bulb. The lamp was faced away from the monitor and subject. Contrast and brightness levels of the monitor were kept approximately the same for all subjects.

Each trial began with a display of the mouse-cursor and the home circle or starting position. The home circle consisted of a $8 \mathrm{~mm}$ diameter red circle with a central circular opening $4 \mathrm{~mm}$ in diameter, and was located centrally at the bottom of the monitor display. In terms of the graphics tablet, this starting position corresponded to a central location approximately $8 \mathrm{~cm}$ from the bottom edge of the tablet's active area.

Once the home circle and cursor appeared on the monitor, the subject moved the cursor to the center of the home circle. The subject was instructed to remain motionless once this had been accomplished. Three seconds following the appearance of the home circle and cursor, a circular target appeared at the upper region of the display. The center of the target was aligned with that of the home circle and was located $130 \mathrm{~mm}$ from the center of the home circle. The subject was free to initiate movement at anytime within a second of the target's appearance, and was instructed not to be concerned with reaction time. The subject was instructed to move as quickly and as accurately as possible, and to ensure that whenever visual feedback was available, the 
cursor should at least be in contact with the target. The subject was also instructed to keep the limb still at the end of the movement until the feedback display appeared.

Two vision conditions were employed. These vision conditions pertain only to vision of the cursor, as all movements were made without vision of the limb. In the Vision-Cursor (VC) condition, the cursor remained visible throughout the movement. In the No Vision-Cursor (NC) condition, the cursor disappeared as soon as its center was moved outside of the small central opening of the home circle. The cursor subsequently reappeared at the end of the trial. At the end of each trial in both vision conditions, a feedback display was provided which showed the home circle, target, and the cursor at its initial position at the time of the target's appearance. In addition, the display showed the cursor at its location at the time the movement ended. Depending upon the nature of the movement, either one, a light-colored cursor, or two, a light and dark-colored cursor was shown. The dual cursor display occurred whenever the executed movement contained a discrete secondary movement which was likely initiated late according to temporal criterions. Thus, subjects received feedback which informed them that an unacceptable delay occurred between the end of 
their initial movement and an attempted correction. ${ }^{4}$ The position of the dark-colored cursor corresponded to the position of the mouse/index finger at the time the subject terminated the arm movement completely. The light-colored cursor indicated where the movement was initially terminated prior to the attempted correction (see section on Data Reduction for a definition of movement termination).

Each subject participated in a single experimental session. At the start of the session, a demonstration of the task and instructions were provided. The subject then performed one block of 12 practice trials for each vision condition. Within each practice block, 4 trials were performed for each target size. Following these two practice blocks, 2 sets of 6 blocks of trials (12 trials/block) were performed. Each set contained one block of each one of the six vision by target size condition combinations. Block/condition order within the first set was randomized with the constraint that three consecutive blocks could not be of the same vision condition. The second set of blocks were randomized in the same fashion, in addition to the constraint that, in combination with the first set of six

4 An unacceptable delay corresponded to a duration exceeding the temporal criterion of $72.0 \mathrm{milliseconds.} \mathrm{This}$ value approximates a lower bound for current estimates of the minimum time to process visual feedback (see Carlton, 1992, for review). 
blocks, no three consecutive blocks of trials could be of the same vision condition.

The first set of 6 blocks were considered as practice at each of the unique vision by target size conditions, and only the last set of 6 blocks were analyzed. In addition, within each block of 12 trials, the first 2 trials were considered as practice and were also not included in the analyses.

\section{Data Reduction}

Following the experimental sessions, and for each trial, the raw displacement data were filtered using a second order dual pass Butterworth filter (low pass cutoff frequency of $6.0 \mathrm{~Hz}$ ). Instantaneous velocity was calculated by differentiating displacement data using a two point central finite difference algorithm. Instantaneous acceleration was calculated by differentiating velocity data using the same algorithm. Since the movement was constrained to the $y$ axis, kinematic data were analyzed with respect to this axis only. An interactive program was developed to identify certain critical points in the displacement, velocity, and acceleration profiles for each trial. The beginning of a movement was defined as the first instance at which the instantaneous velocity was greater than or equal to $1.0 \mathrm{~mm} / \mathrm{s}$. The end of the movement was defined as the first instance at which instantaneous velocity fell within $+/-1.0 \mathrm{~mm} / \mathrm{s}$, and 
remained within these thresholds for a period greater than 10 sampling frames $(72.0 \mathrm{~ms})$. If a subsequent reacceleration (an increase in velocity in either the positive or negative direction and exceeding threshold values) occurred prior to the end of the 10 frame window following the temporarily defined movement end, then depending upon the nature of this reacceleration, movement end was redefined. If the increase in velocity was equal to or exceeded a duration of $72 \mathrm{~ms}$ (10 frames), then a search for a new movement end was done, according to the same criterion and procedures above. However, if the duration was less than 10 frames and velocity returned to within threshold values, then movement end was not redefined and was taken as the previously defined endpoint. Movement time was therefore calculated as the number of frames between the defined start and end of the movement, divided by the sampling frequency. Constant error was calculated as the signed difference between the $\mathrm{Y}$ coordinates of the center of the cursor and the center of the target.

\section{Movement Modifications}

Critical points marked on the velocity and acceleration profiles aided in the identification of kinematic indices of movement modifications. Four types of modifications were defined and enumerated from the kinematic data for each trial. These were significant deviations in 
the acceleration profile prior to peak velocity and following peak velocity, negative to positive transitions of the acceleration profile in the period between peak velocity and the end of the movement, and reversals of the direction of movement.

To identify significant deviations in the acceleration trace in the period between the start of the movement and peak velocity (SDPV), a search for a reversal point, other than that of peak acceleration was first performed. If such a reversal point was present, then the second reversal point following the start of the movement was marked. For a significant deviation to be present, two criterions had to be satisfied. First, the amplitude between this marked reversal and the consequent one had to satisfy the predefined amplitude criterion. 5 second, the duration between this marked reversal and the point at which the magnitude of acceleration fell below that value at the marked reversal had to equal or exceed the temporal criterion. ${ }^{6}$ Any subsequent deviations were defined in the same manner. For additional details and illustrations of this procedure, see Figures 4 and 5 .

5 The amplitude criterion was a value corresponding to ten percent of the greatest absolute magnitude in acceleration.

6 The temporal criterion was constant at 72.0 milliseconds. Although this value was arrived at arbitrarily, it is consistent with estimates of other investigators (e.g., see van Donkelaar \& Franks, 1991). 

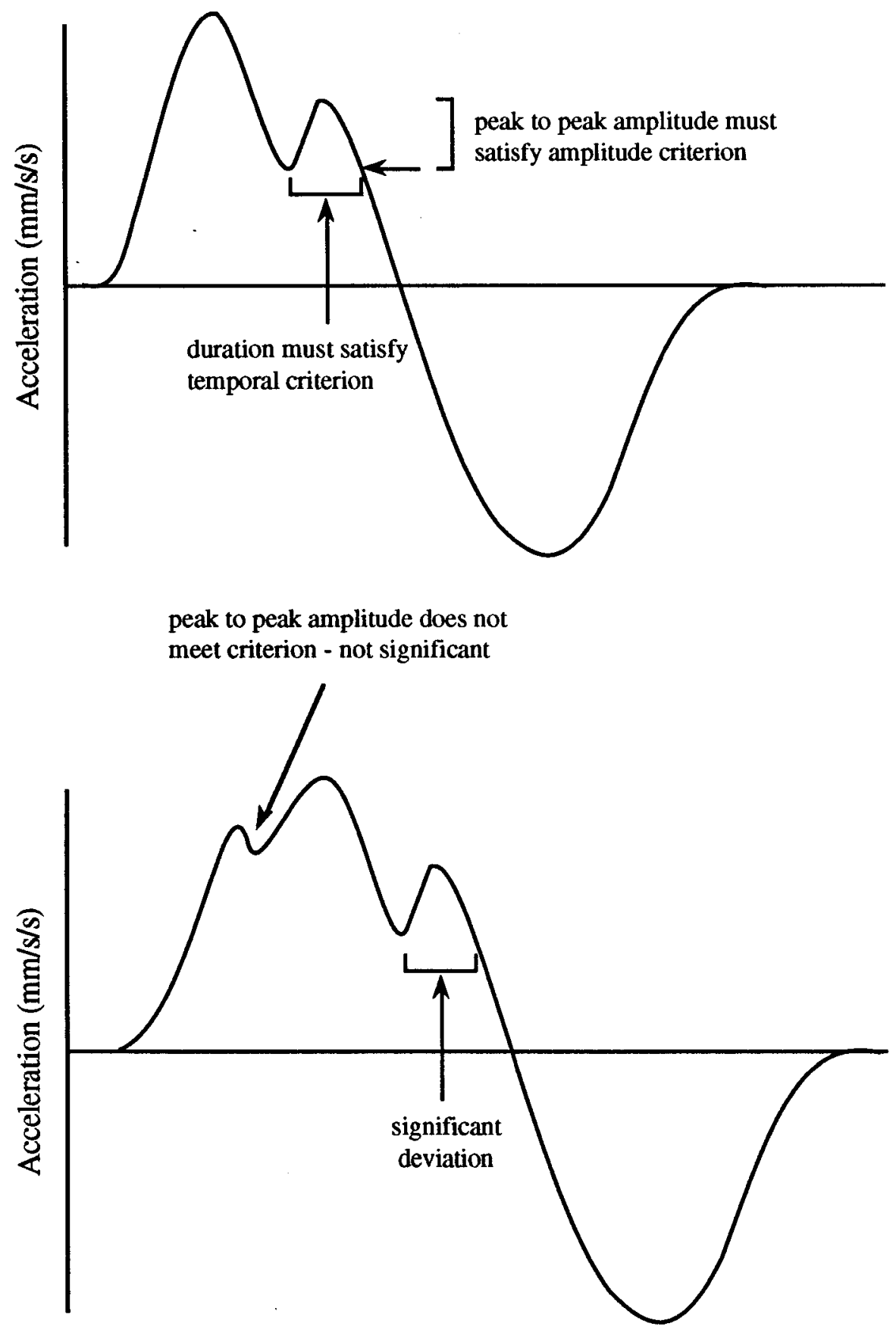

Figure 4. Schematic illustrations of the definition of a significant deviation in the acceleration profile prior to time of peak velocity. 


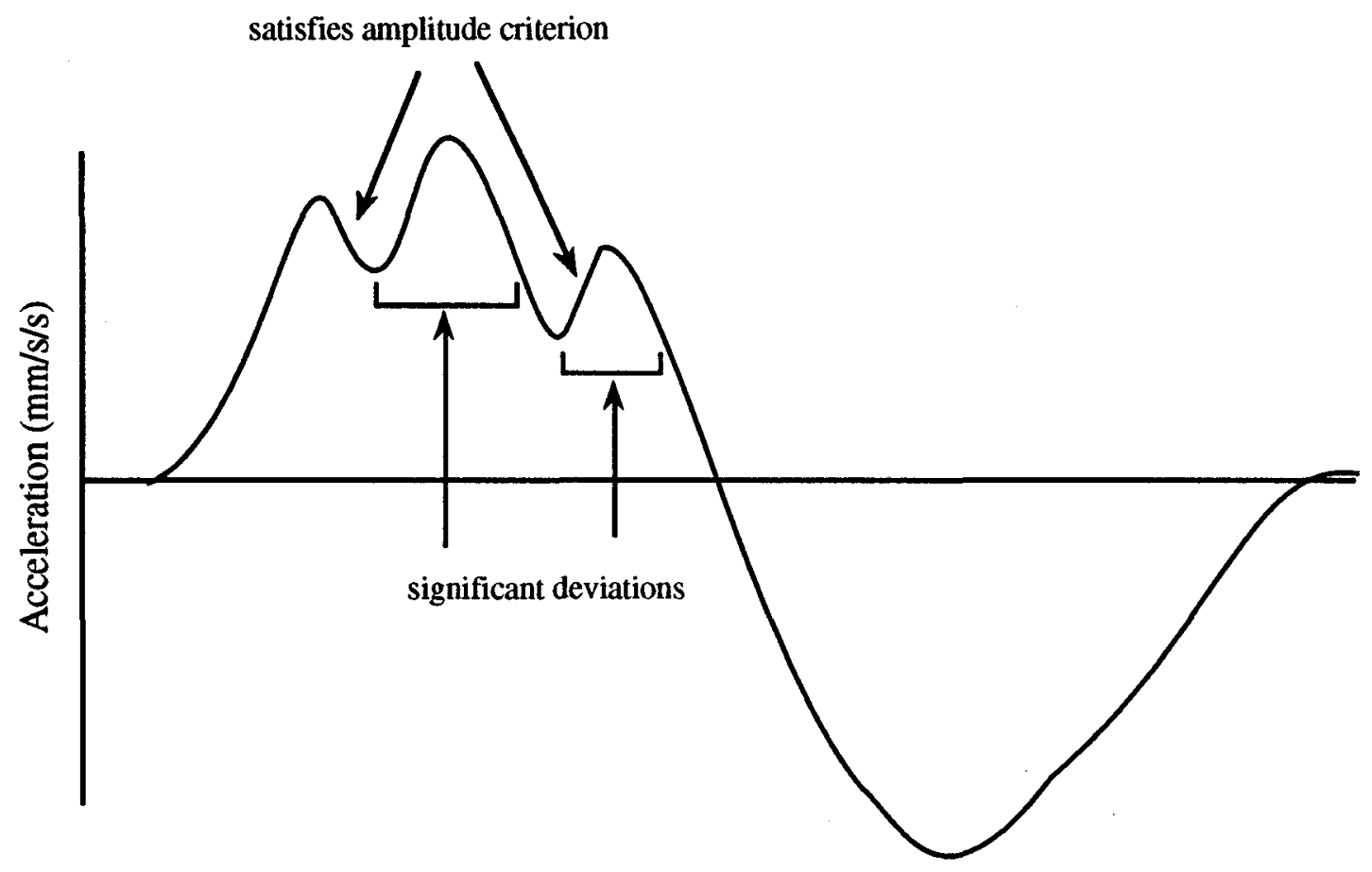

Figure 5. Diagrammatic example of an acceleration profile with two significant deviations prior to the time of peak velocity. 
To identify significant deviations in the period between peak velocity and the end of the movement (PVSD), a search was again performed for any reversal points other than that of peak negative acceleration. If such a reversal point was present, then the second reversal point following the point of peak velocity was marked. For a significant deviation to be present, two criterions had to be satisfied. First, the amplitude between this marked reversal and the consequent one had to satisfy the amplitude criterion. Second, the duration between this marked reversal, and the next reversal point (or the end of the movement) had to satisfy the temporal criterion. Other deviations were defined in the same manner. Figures 6 and 7 provide additional details of this procedure.

To identify zero crossings in the acceleration profile following peak velocity $(\mathrm{ZC})$, a search was made for a negative to positive transition in acceleration. For any such transitions to be defined as a zero crossing, the "peak" in the velocity profile associated with this transition had to satisfy two criterions. First, the amplitude of the velocity peak, measured from the relative velocity minimum to this peak, had to equal or exceed $5.0 \mathrm{~mm} / \mathrm{s}$. Second, the duration between the start of this "reacceleration" in velocity and the point at which the velocity value fell below that value at the start had to satisfy the temporal 

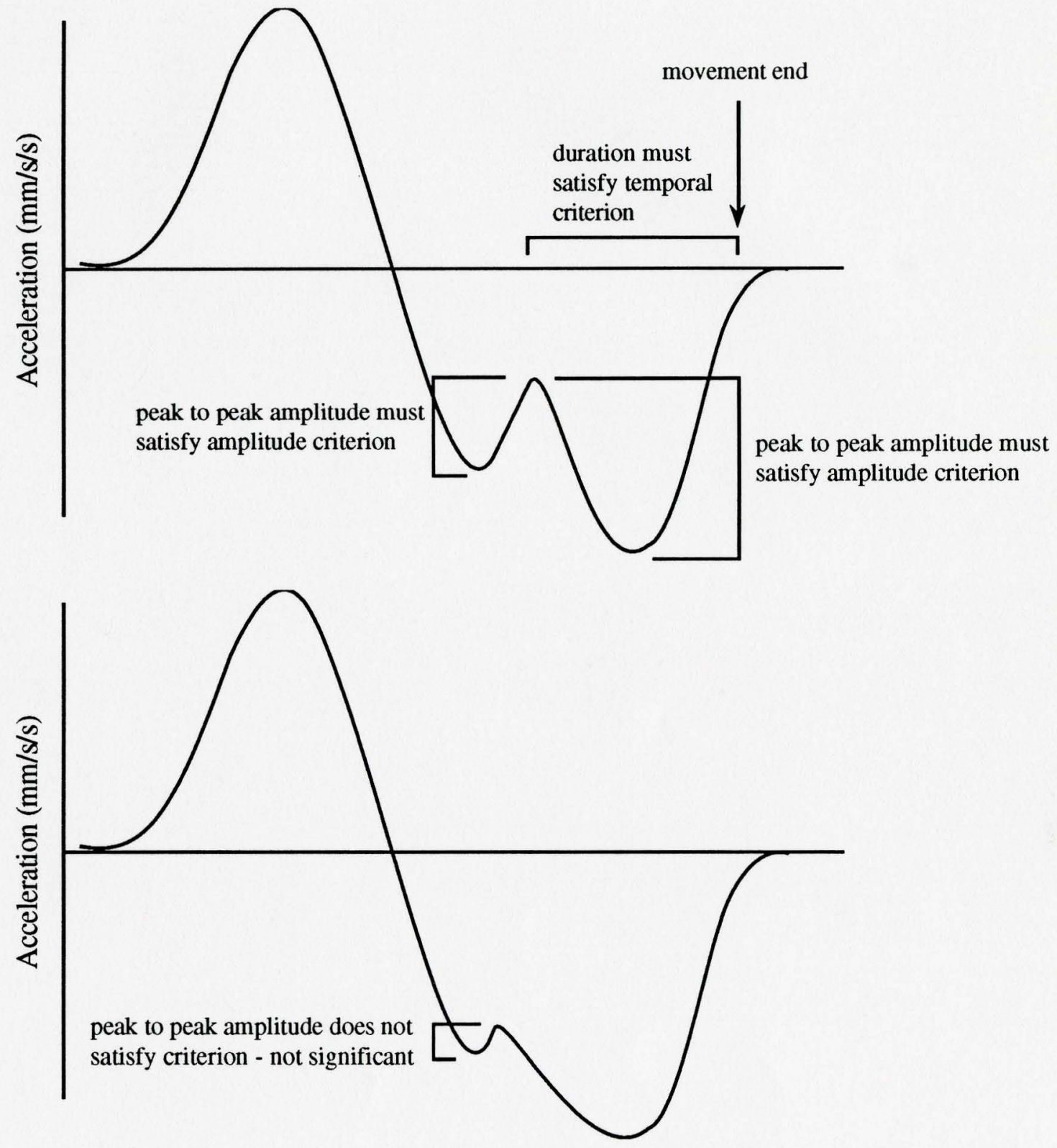

Figure 6. Schematic illustrations of the definition of a significant deviation in the acceleration profile following the time of peak velocity 


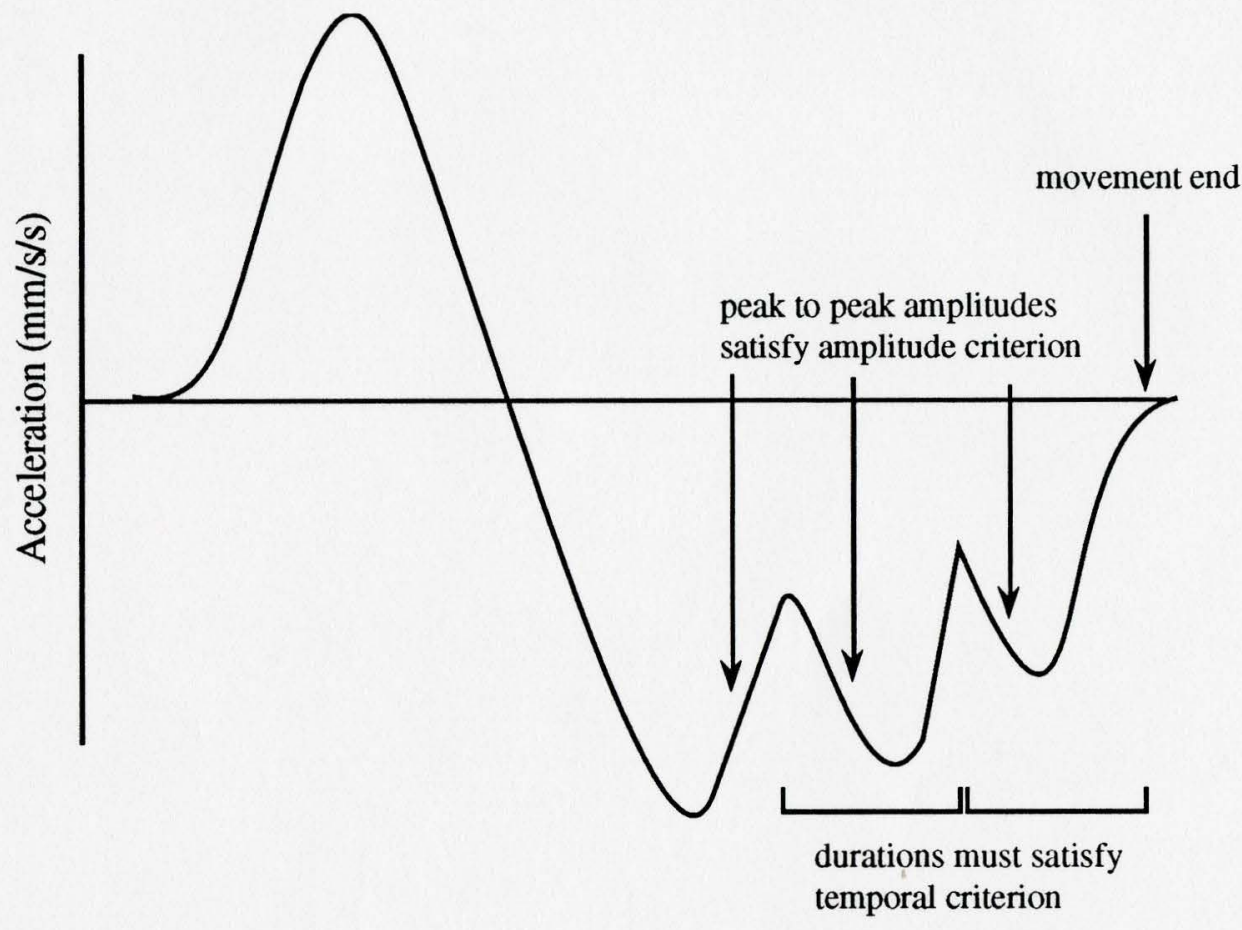

Fiqure 7. Schematic example of an acceleration profile containing two significant deviations following time of peak velocity. 
criterion See Figure 8 for an illustration and additional details.

Reversals in the movement (REVS) were identified from a change in the sign of velocity for the movement reversal or negative velocity to be significant, the absolute magnitude of negative velocity had to remain above the velocity threshold $(10 \mathrm{~mm} / \mathrm{s}$ for a minimum duration equal to the temporal criterion (see Figure 9

Figures 4 to 9 provide illustrative schematic examples of these analytic procedures plots of actual kinematic profiles are contained in Appendix B Appendix A provides a summary of terms and their definitions

For each trial, performance data were available relating to movement time, constant error, and variable error. Kinematic data were available relating to peak velocity of the movement, time to peak velocity as a proportion of the movement time, and the displacement at the time of peak velocity expressed as a proportion of target distance. In addition, data pertaining to movement modifications included the number of significant deviations in the acceleration profile prior to peak velocity and following peak velocity, the number of negative to positive zero crossings of the acceleration profile following peak velocity, and movement reversals 


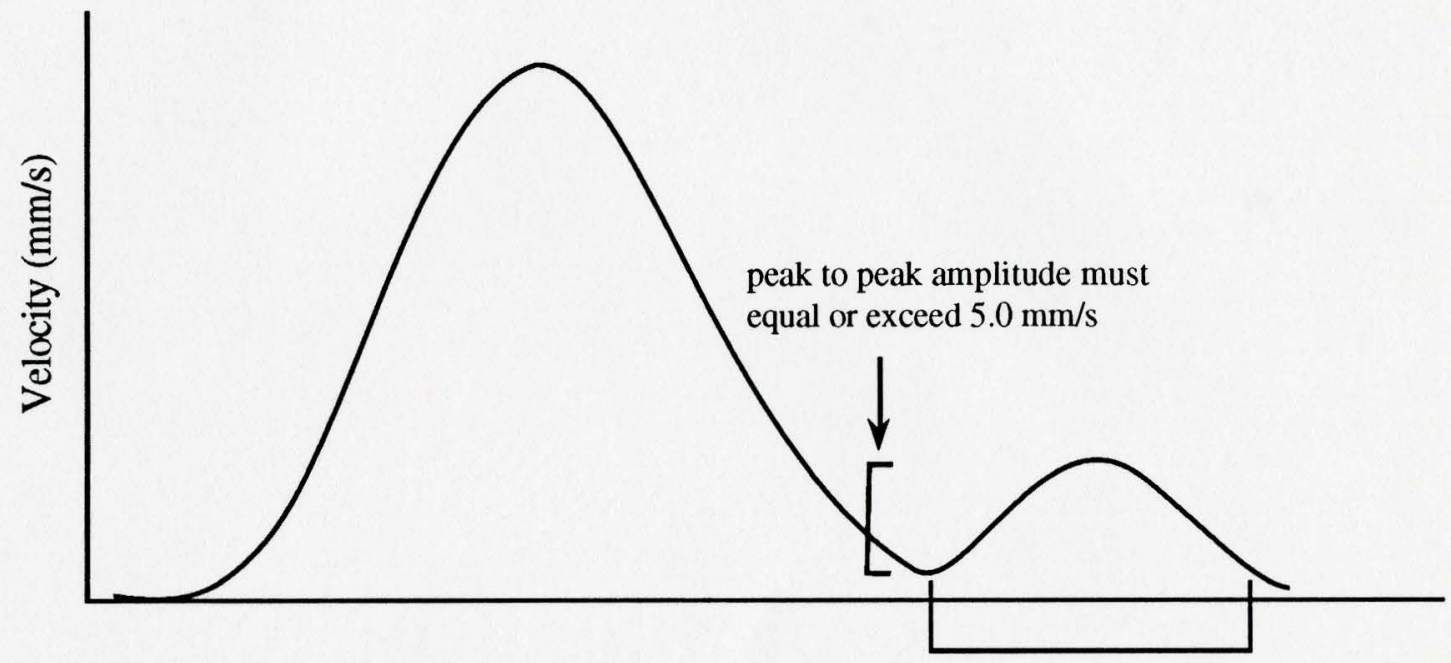
duration must satisfy

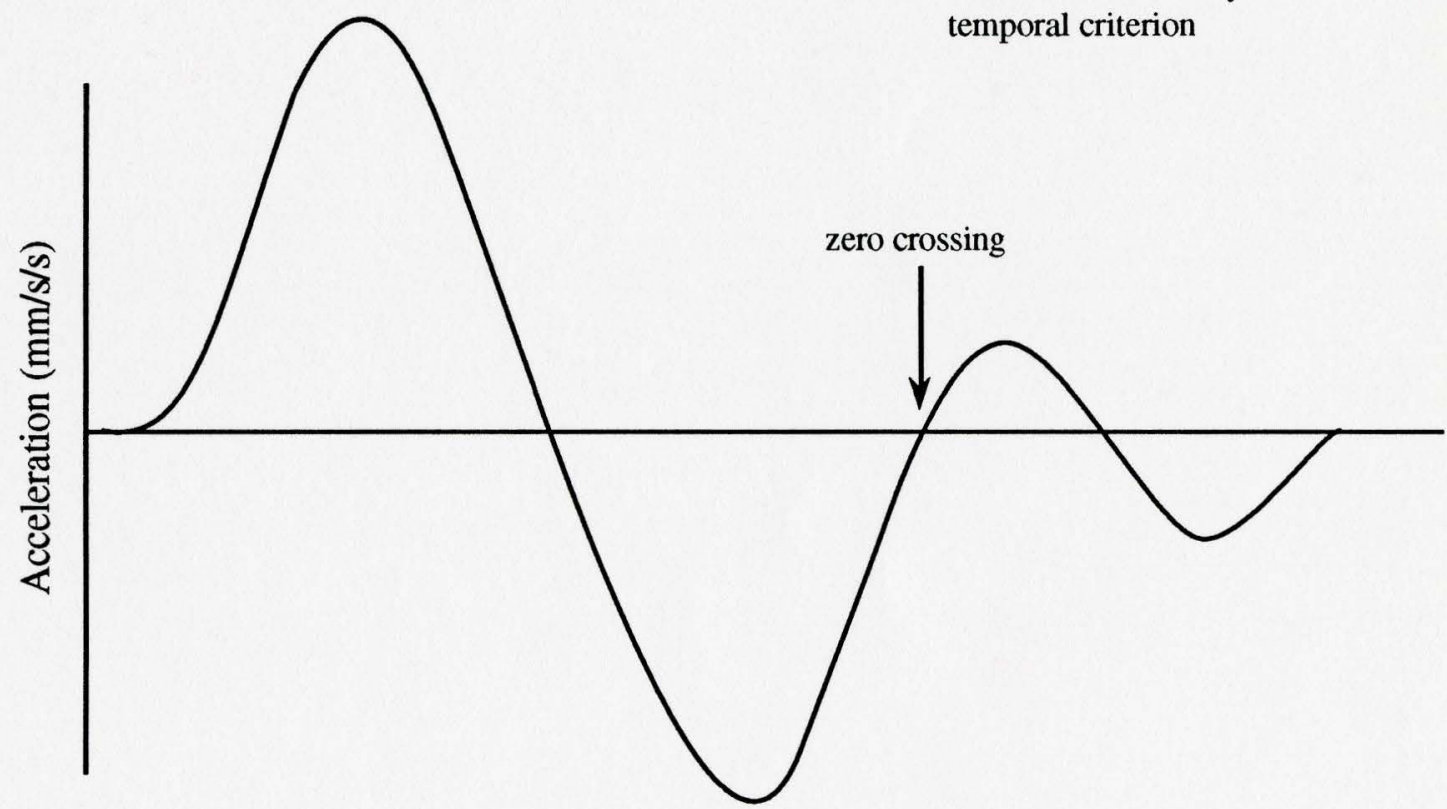

Figure 8 Schematic illustration of a zero crossing defined from the velocity and acceleration profiles 


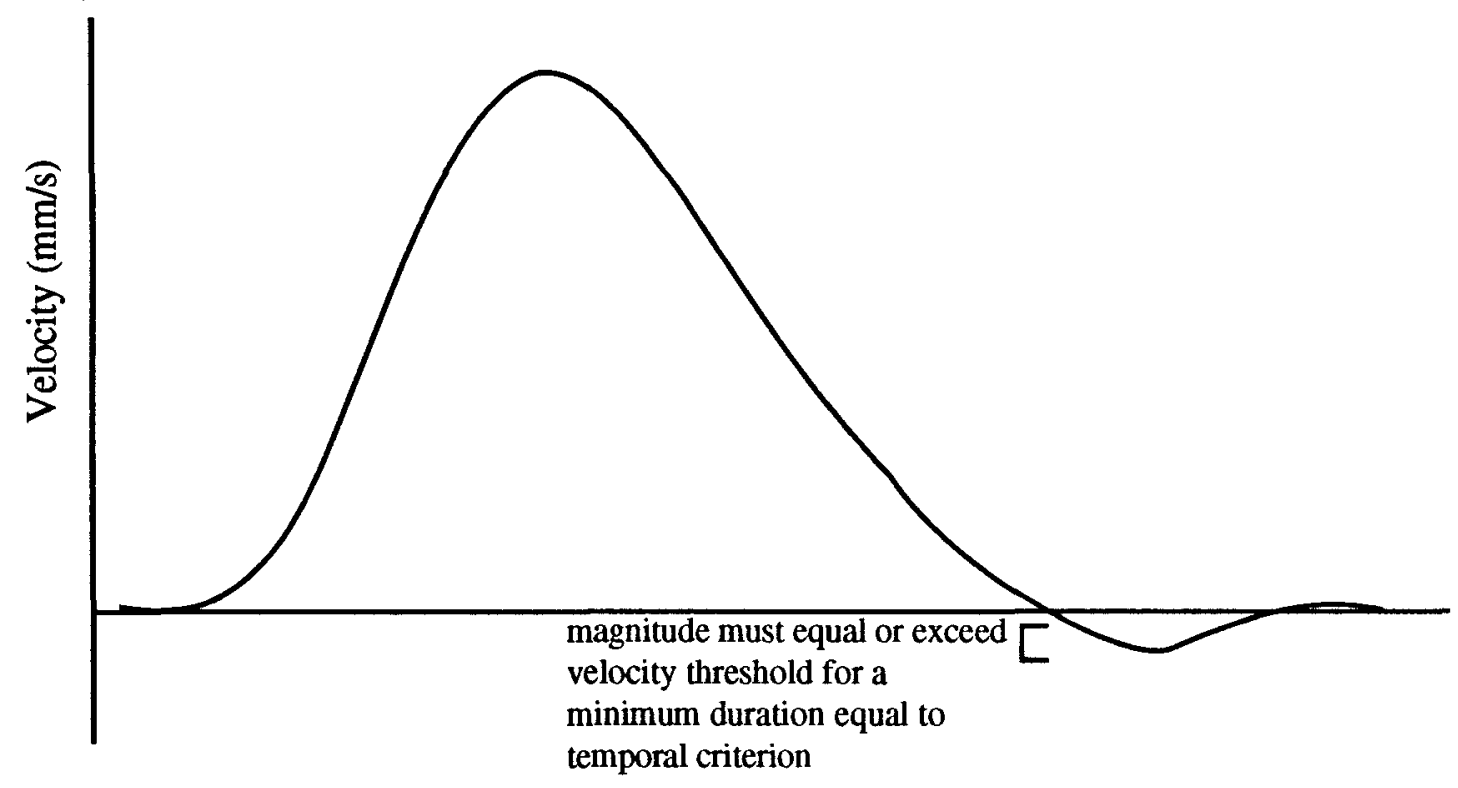

Eiqure 9. Illustration of the definition of a movement reversal. 
Results

Mean values for each of the dependent variables were obtained from cells formed from a combination of the independent factors, with each mean value calculated from 10 trials. 7 Whenever possible, the dependent variables were analyzed separately using a $2 \times 3$ (Vision condition $\mathrm{x}$ target size) repeated measures analysis of variance for those variables for which parametric analyses were not possible, non-parametric analyses were performed

\section{Performance Measures}

Analysis of movement time revealed a main effect for Vision condition, $\underline{E}(1,9)=1096, \underline{p}<01$, and an effect for target size which approached conventional levels of significance, $\underline{E}(2,18=350, \underline{\underline{D}}=.051$ Consistent with previous studies which have shown faster movement times in situations of degraded visual conditions (e g, Carson et al, 1990, in press; Elliott et al, 1991; Elliott \& Madalena, 1987; Wallace \& Newell, 1983; cf Meyer et al, 1988; Prablanc et al., 1979a, subjects executed their movements more quickly in the No Vision condition compared to the Vision-cursor condition (see Figure 10 As would be expected (e g , Fitts, 1954), movement times also tended to

7 For the movement modification variables, the sums, rather than the means, were used for analysis This was done merely for convenience and does not affect the outcome of the analyses 
decrease with increasing target size and hence, decreasing index of difficulty

Analysis of constant error revealed no significant effects. Based on previous work (e g, Elliott et al, 1991; Prablanc et al, 1979a, 1986, it was expected that subjects would tend to undershoot the target center in the No Vision condition The absence of any effects for constant error is more supportive of Meyer et al 1988, whose model predicts that movement endpoints will be distributed about the center of the target and will not vary with vision condition The variable error analysis indicated that subjects were more consistent in the Vision-cursor condition compared to the No Vision condition, $\underline{\mathrm{F}} 1,9)=9163, \underline{\mathrm{p}}<001$ This vision main effect accounted for a relatively large proportion of the variance $\omega^{2}=512$ The effect for vision was mediated by target size, indicated by the interaction of vision and target size, $\underline{F} 2,18=4.53, \underline{\mathrm{p}}<$ .05. Figure 11 illustrates that with vision of the cursor, subjects' movement endpoints became more variable with increasing target size, indicating that subjects made use of the larger target region e $g$, larger effective target widths). When vision of the cursor was not available, however, this effect was not apparent 


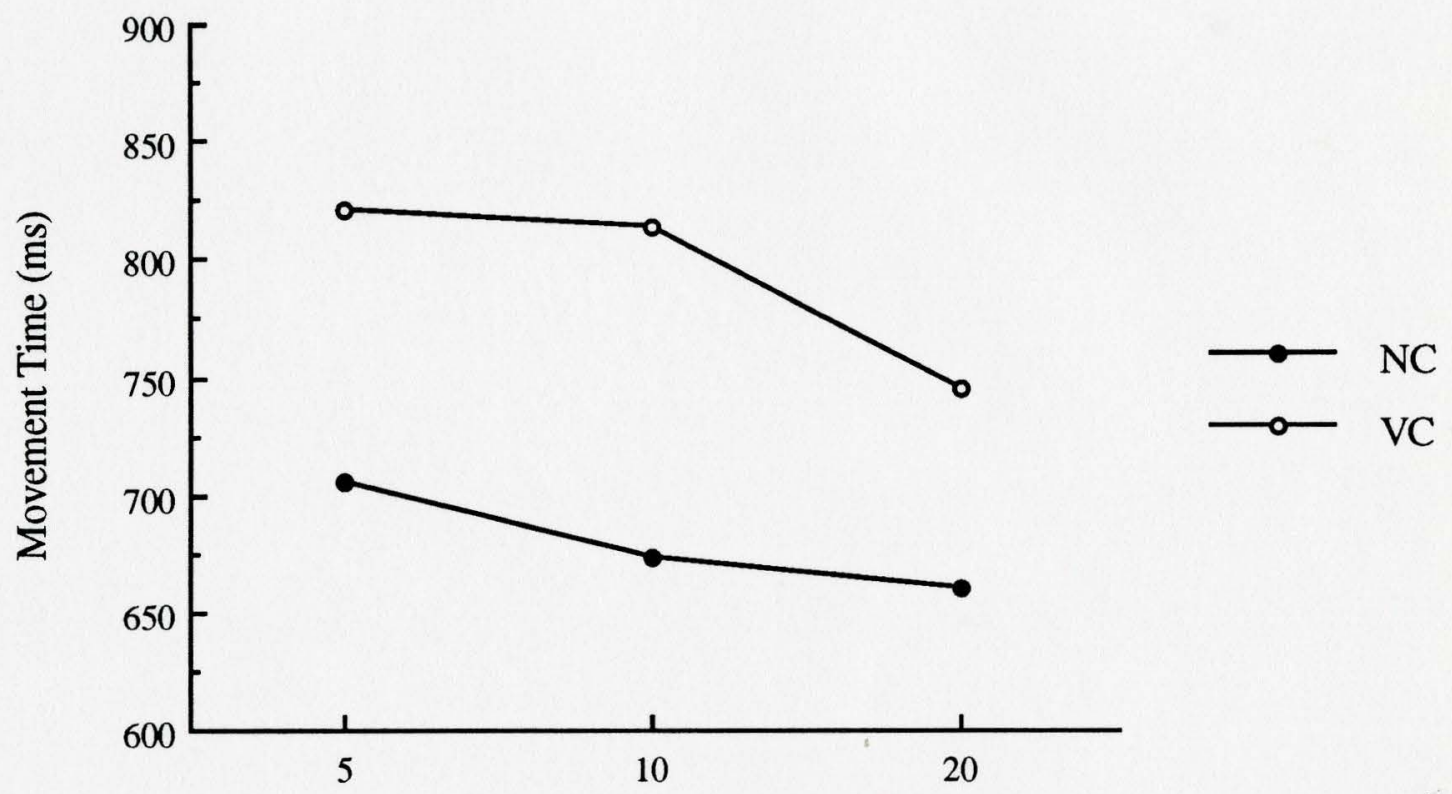

Target Size $(\mathrm{mm})$

Figure 10. Experiment 1 Movement time (ms) as a function of Vision condition and target size (mm 


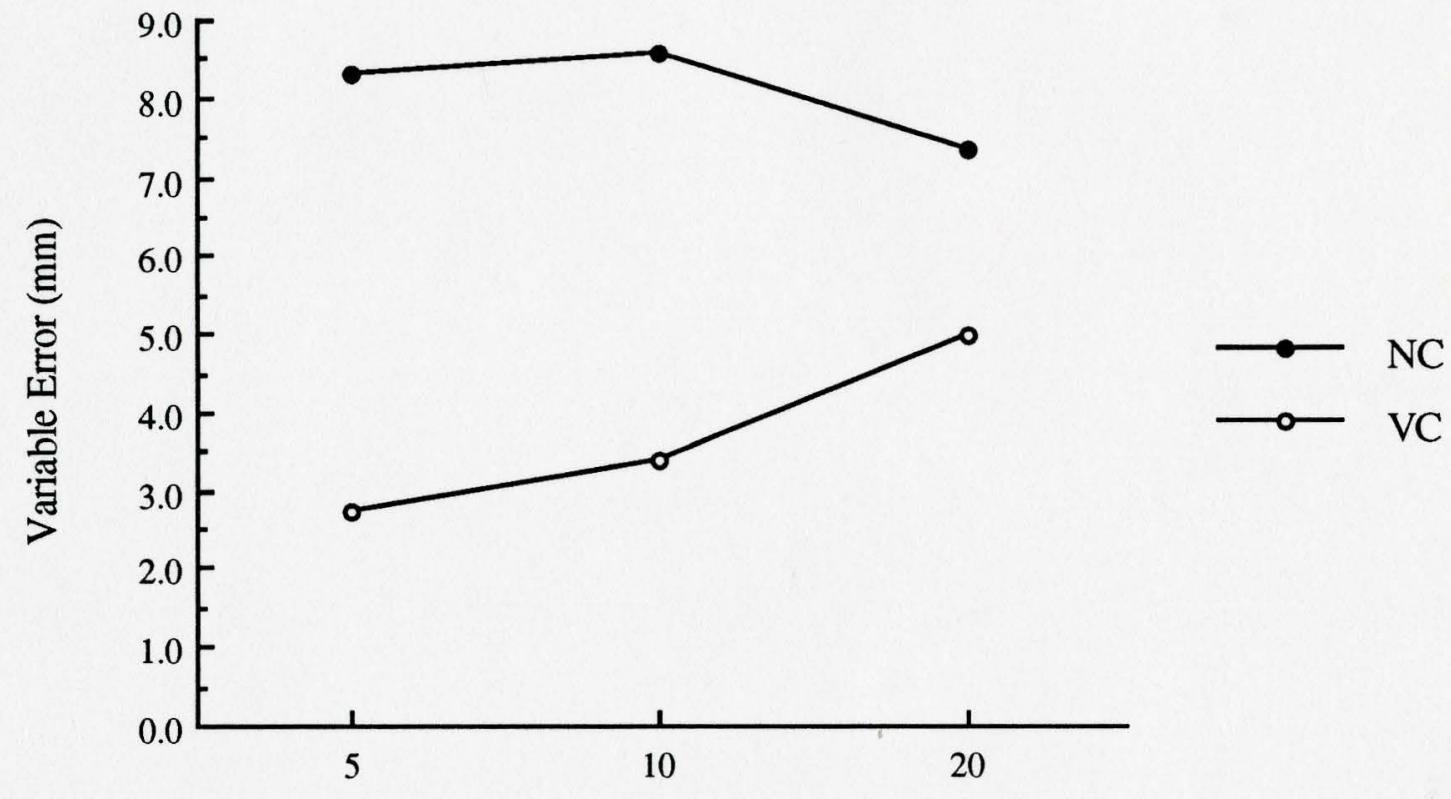

Target Size $(\mathrm{mm})$

Figure 11 Experiment 1: Variable error (mm) as a function of Vision condition and target size (mm) 


\section{Kinematic Measures}

While the kinematic analyses revealed no significant effects for peak velocity (cf., Mackenzie et al., 1987; Milner \& Ijaz, 1990), a main effect for vision condition was found for both the time to peak velocity as a proportion of movement time (TPV/MT), $\underline{\mathrm{E}}\left(1,9=7204, \underline{\mathrm{p}}<001,\left(\omega^{2}=\right.\right.$ .665), and the distance travelled at the time of peak velocity as a proportion of the target distance (PVDISP/DIST), $\underline{\mathbf{F}}(1,9)=3960, \underline{\mathrm{D}}<.001$ As depicted in Figure 12, subjects attained peak velocity relatively earlier when vision of the cursor was available, and consequently spent a greater proportion of their movements in the deceleration phase Presumably, this allowed time to utilize available visual information and for modifications to be made to the movement if necessary The absence of a target size effect is in contrast with previous studies which have shown that increasing demands for accuracy results in the prolongation of the deceleration phase of the movement (Langolf et al., 1976; Mackenzie et al , 1987; Milner \& Ijaz, 1990; Soechting, 1984; Todor \& Cisneros, 1985

Since peak velocity was reached proportionally earlier in the Vision-cursor condition, subjects also travelled a lesser proportion of the trajectory at the time of peak velocity in this condition (see Figure 13) The absence of a target size effect for PVDISP/DIST further 


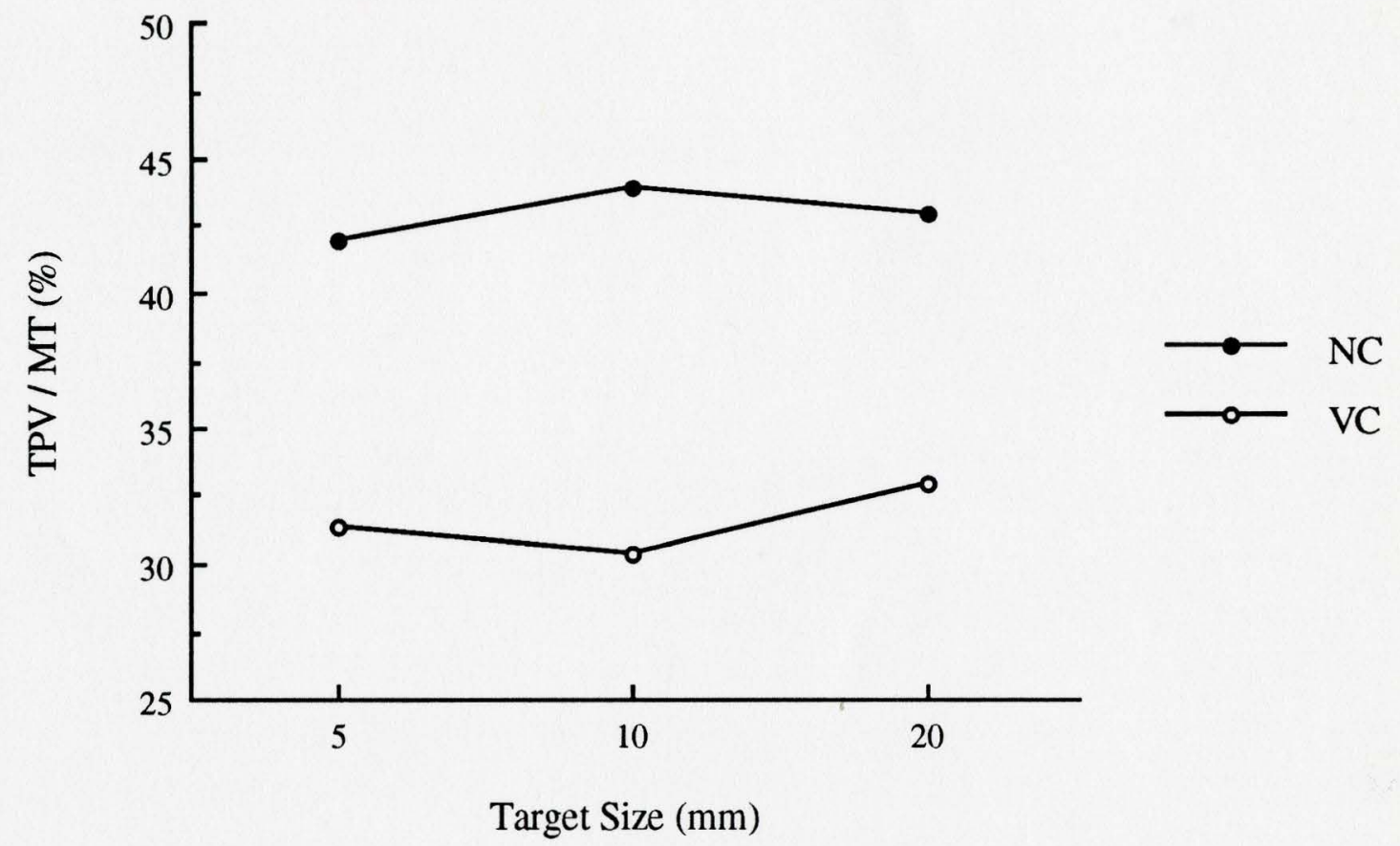

Figure 12 Experiment 1 Time to peak velocity, expressed as a proportion of movement time ( $\%$, as a function of Vision condition and target size $\mathrm{mm}$ 
indicates that this initial displacement was independent of target size, a finding which replicates Mackenzie et al (1987). The overall mean of 44 is comparable to that (40) found by these previous authors

The longer deceleration phase characteristic of accurate visually guided movements has traditionally been presumed to allow time for the visual detection and reduction of errors in the movement see Jeannerod, 1988, for review I therefore examined whether the kinematics of this portion of the movement reflected the active process thought to occur in bringing the hand/cursor to the target. In addition, I searched for evidence of modifications which occurred during the movement. Examination of the variables reflecting movement modifications revealed that with the exception of the variable PVSD, the distributions of the other three dependent variables, SDPV, ZC, and REVS, were markedly skewed. Therefore, these latter variables were analysed using friedman analysis of variance by ranks

A 2 × 3 repeated measures analysis of variance performed on the number of significant deviations in the acceleration profile following peak velocity PVSD revealed a main effect for vision condition, $\underline{\mathrm{F}} 1,9=717, \underline{\mathrm{p}}<.025$ Figure 14 illustrates the greater number of deviations in the acceleration profiles of movements made with vision of the cursor available This finding concurs with that of carson 


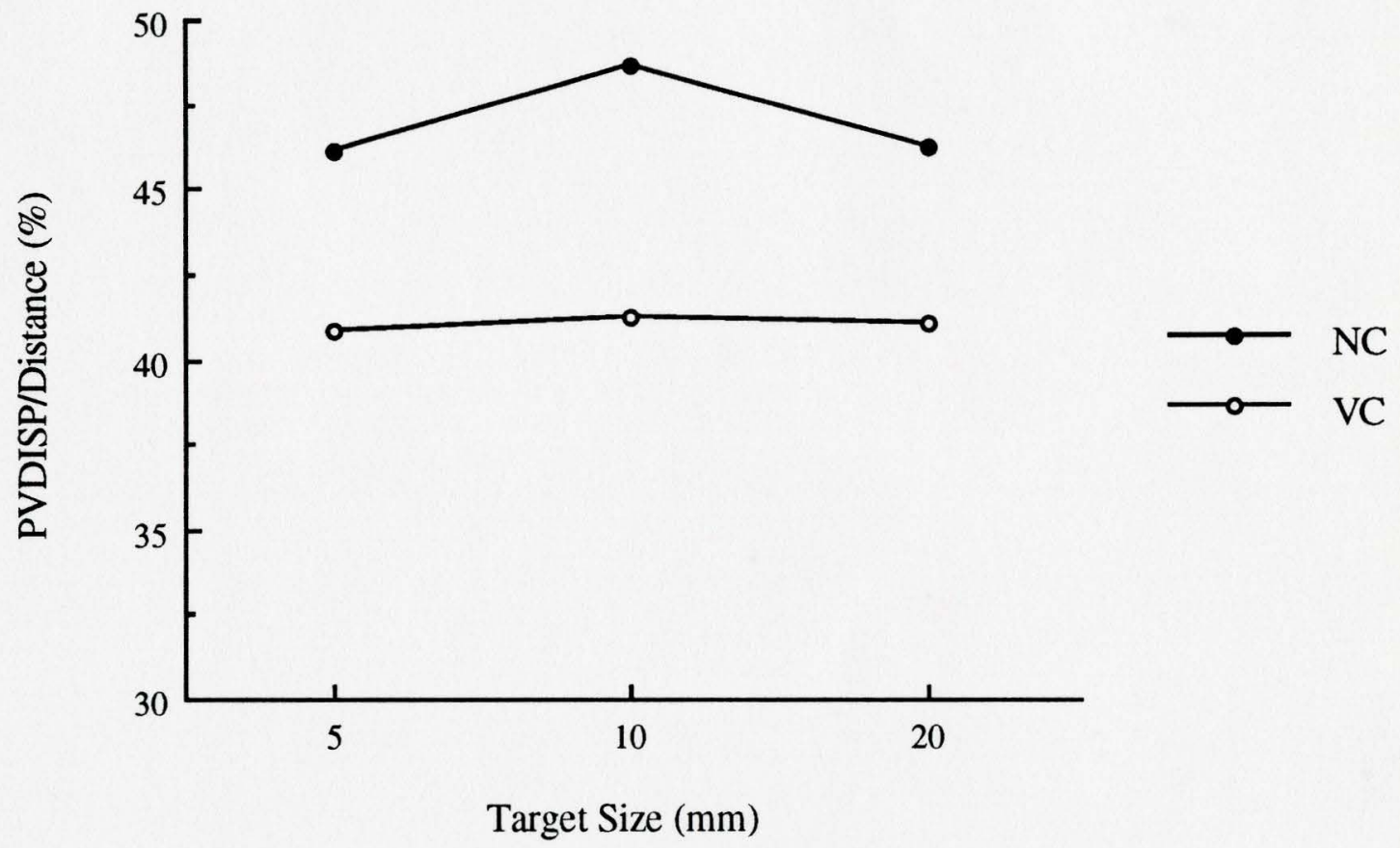

Figure 13 Experiment 1: Displacement at time of peak velocity, expressed as a proportion of target distance \% as a function of Vision condition and target size (mm) 
et al. (in press).

The initial analysis of positive zero crossings ( $\mathrm{ZC}$ ) using a Friedman anova revealed a significant effect amongst the six vision $x$ target size conditions, $\chi^{2}(5, N=10)=$ $31.89, \mathrm{D}<.001$. In order to determine the source of this effect, further tests were performed to examine specific questions. The means for zero crossings were collapsed across Vision condition and a test for an effect of target size was performed. This comparison failed to yield significance. To test for an effect for Vision condition, means were collapsed across target size. The Friedman anova revealed a significant effect for Vision condition, $\chi^{2}(1, \mathrm{~N}=$ 10) $=10.00, \mathrm{Q}<.005$, thus indicating that the difference found in the initial six condition analysis was likely due to the influence of vision. Specifically, subjects exhibited a greater number of secondary accelerations in their movements when vision was available (see Table 1 ).

Initial six level Friedman analyses performed on the number of significant deviations in the acceleration profile prior to peak velocity (SDPV) and on the number of movement reversals (REVS) failed to establish significance. Therefore, no further tests were performed on these variables. Thus, of the four variables relating to movement modifications, two, the number of significant deviations following peak velocity and zero crossings, were found to 


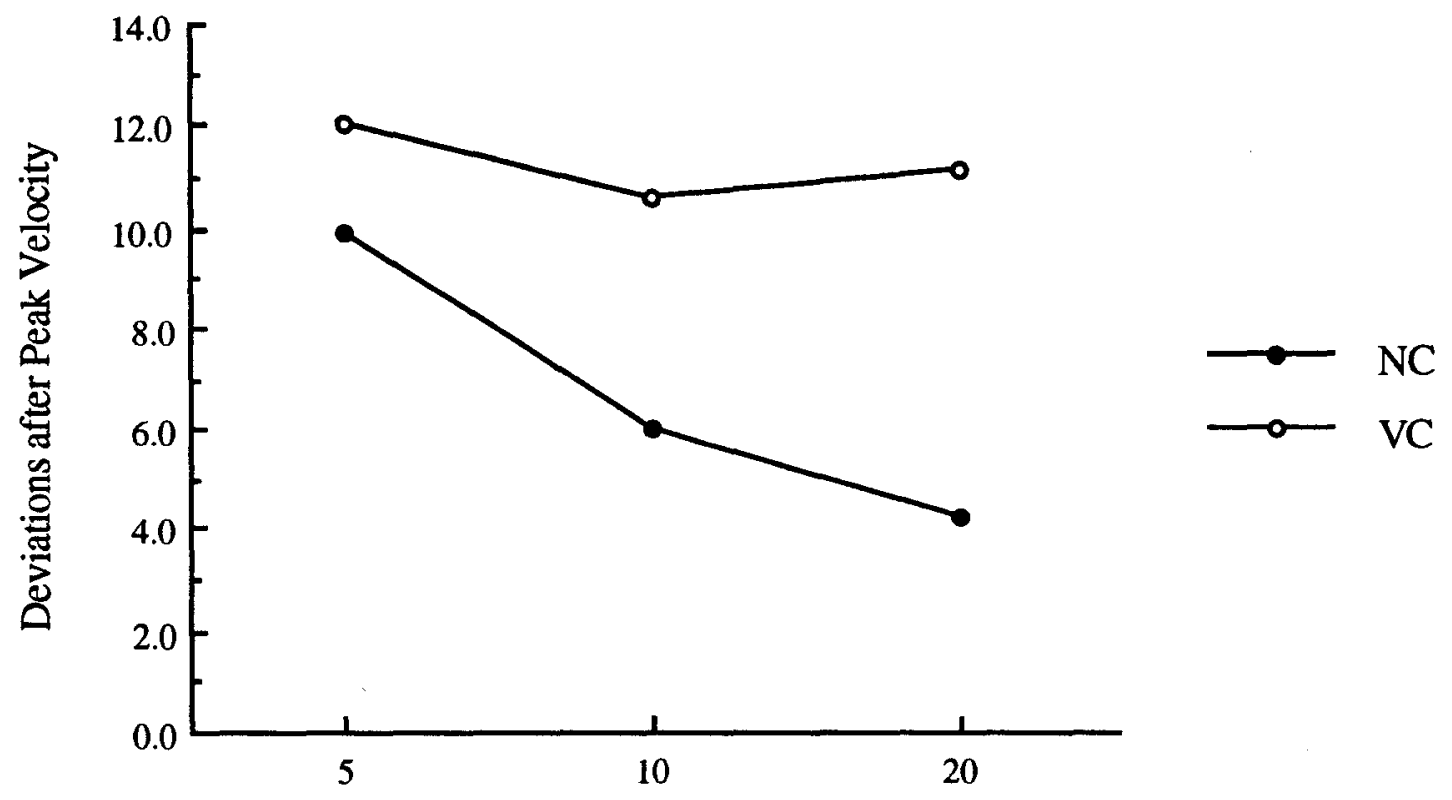

Target Size (mm)

Eigure 14. Experiment 1: Mean sum of significant deviations in the acceleration profile following time of peak velocity as a function of Vision condition and target size (mm). 
Table 1

Mean sum of zero crossings in the Acceleration Profile as a Function of Vision condition and Target size

Target Size

Vision Condition

$5 \mathrm{~mm} \quad 10 \mathrm{~mm} \quad 20 \mathrm{~mm}$

Vision-Cursor

4.50

6.10

2.80

No Vision-Cursor

0.40

0.10

0.50 
vary as a function of vision condition. Presumably, this reflects a greater number of modifications in movements made under visual guidance, and these modifications, in turn, contribute to the better performance observed. This particular finding is in direct opposition with those of previous investigators (e.g., Carson et al., 1992; Elliott et al., 1991; Meyer et al., 1988) who have found that performance advantages associated with visual feedback were independent of the number of corrections in the movement trajectory. However, the finding once again is consistent with Carson et al. (in press).

Correlational Analyses

To examine whether any relationships existed between selected performance and kinematic variables, a number of correlational analyses were performed. Correlations were performed between movement time (MT) and the total number of modifications (MODS), between the time from peak velocity to the end of the movement (TPV-END) and absolute error (AE), and the total number of modifications and absolute error. For each analysis, within-subject correlation coefficients were derived for each subject for each condition. These coefficients were then transformed into Fisher $z$-scores and analysed using a 2 Vision condition $\times 3$ target size repeated measures analysis of variance. 
The overall positive relation between MT and MODS (grand mean $\mathrm{z}=0.85, \underline{\mathrm{Q}}<.001$ ) indicated that increased movement durations were associated with more modifications to the trajectory. Moreover, this relation was more pronounced when vision of the cursor was available $(z=1.01)$ compared to when it was not $(z=0.68), \underline{E}(1,9)=5.72, \underline{\mathrm{D}}<.05$.

There was an overall negative relation between TPVEND and $\mathrm{AE}$ (grand mean $\mathrm{z}=-0.19, \underline{\mathrm{Q}}<.01$ ), indicating that movements with longer deceleration phases were associated with less error. This relation was primarily present in the Vision-cursor condition $(z=-0.39)$ and essentially absent in the No Vision condition $(z=-0.01), E(1,9)=10.89, \mathbb{Q}<.01$. In addition, the extent of the negative relation was greater for the smallest target size $(5 \mathrm{~mm} \mathrm{z}=-0.38 ; 10 \mathrm{~mm} \mathrm{z}=-$ $0.17 ; 20 \mathrm{~mm} z=-0.02), \underline{E}(2,18)=4.31, \underline{Q}<.05$.

The relation between MODS and AE were found to be independent of both Vision condition and target size. There was, however, an overall negative relation between the two variables (grand mean $\mathrm{z}=-0.20, \mathrm{p}<.01$ ). Thus, it appears that better accuracy was achieved when modifications were made to the movement.

\section{Discussion}

The primary purpose of this experiment was to examine the influence of varying visual condition and target size on manual aiming performance and aiming trajectories. of 
interest were the relationships between the performance and kinematic measures, and how these indices varied with visual condition.

The availability of visual information proved to be the predominant influence on the performance and kinematic measures. In contrast, target size was not of major significance, having only a small influence upon movement time and the relationship between TPV-END and absolute error, and mediating the effect of vision on variable error. The minimal influence of target size in this experiment may have been due to the limited number of target widths employed. Increasing demands for accuracy by decreasing target size has previously been found to affect both the magnitude of peak velocity and the duration of the decelerative phase of aiming movements (e.g., Langolf et al., 1976; Mackenzie et al., 1987; Milner \& Ijaz, 1990; Soechting, 1984).

Some support was found for the optimized dual submovement model (Meyer et al., 1988) with respect to predictions concerning the distribution of movement endpoints. According to Meyer et al. (1988), movement endpoints should be distributed normally or symmetrically about the center of the target (i.e., mean constant error of zero) and that this distribution should be independent of visual conditions. The findings for constant error in the present experiment concur with these predictions (cf. Elliott 
\& Madalena, 1987; Elliott et al., 1991; Prablanc et al., 1979a, 1986). Specifically, subjects did not exhibit any consistent tendencies to undershoot or overshoot the center of the target region. Moreover, endpoint bias did not vary with vision condition. It may be that the continual availability of vision of the target eliminated or reduced any pattern for either overshooting or undershooting.

A significant influence of vision condition was evident in the variability of movement endpoints, accounting for half of the variable error variance. With vision of the cursor available, subjects were able to decrease their endpoint variability by $50 \%$, reflecting the beneficial effects of vision. Given this performance advantage which presumably is related to the availability of visual information, specific questions can be examined. Specifically, in what manner was vision utilized, or what underlying processes may vision have subserved, which resulted in superior performance? Changes in the kinematics of the limb movements were evident between the two visual conditions. It is of interest to determine whether these differences were reflective of, and accounted for, the differences in aiming variability.

In agreement with others (e.g., Elliott et al., 1991), movements were of longer duration when vision was available (cf. Meyer et al., 1988). Moreover, the effect of 
the visual manipulation on movement duration was primarily manifested as prolonged deceleration phases. While these movement time results are consistent with those of previous work (e.g., Elliott \& Madalena, 1987; Elliott et al., 1991), the underlying reasons probably are in opposition. It has been suggested that shorter movement times in No Vision conditions are a result of the subject's attempts to acquire the target before the representation of the target position has had time to decay (see Elliott, 1990, 1992; Elliott \& Madalena, 1987; Elliott et al., 1991). In these previous studies, vision of the target (and limb) was eliminated either coincident with, or prior to, the onset of the movement. In the present study, vision of the target was continuously available, even in the No vision condition. Only dynamic visual information pertaining to the position of the limb/cursor was absent. Subjects therefore did not have to be dependent upon a representation of the target. Thus, it seems unlikely that the above explanation would be a valid account for the present results. In support of this, the correlational analyses demonstrated no relationship between the duration of the decelerative phase of the movement and terminal accuracy when vision of the cursor was absent (cf. Elliott et al., 1991). A positive relation might have been expected if longer movement durations were accompanied by a decrease in accuracy, due, presumably, to the deterioration 
of a target representation. Indeed, when target information is present, movement time differences between Full Vision and No Vision conditions due to the aforementioned strategy, may essentially be eliminated (e.g., Elliott, 1988, 1990; Elliott et al., 1991). In the present experiment, the shorter movement times under No Vision conditions, or alternatively, the longer movement times in Vision-cursor conditions, must therefore be a consequence of some other underlying process or movement strategy.

Traditional closed-1oop models of visual-motor control assume that vision is used to detect and reduce discrepancies between the position of the hand and target (e.g., Keele, 1968; 1981). Given that corrective adjustments to the movement trajectory are based upon visual feedback (e.g., Crossman \& Goodeve, 1963/1983; Howarth et al., 1971; Woodworth, 1899), this implies that these corrections should not be evident in movements made without visual feedback (Elliott et al., 1991). Therefore, shorter movement durations for movements made under No Vision conditions may be a consequence of an absence (or a lesser number) of time consuming modifications to the trajectory. Conversely, longer movement times under Full Vision conditions may be a result of the presence of these modifications. The observation of a greater number of modifications in movements made under the Vision-cursor condition is consistent with 
this explanation Thus, the longer movement times may be due to the presence of visual feedback-based modifications The positive relationship between movement time and the number of modifications suggests that these adjustments are time consuming 8 Moreover, the influence of vision upon this relation suggests that vision plays a role in the active regulation of the movement Additionally, the present movement time findings conflict with the assumptions of the dual submovement model (Meyer et al, 1988 Meyer et al (1988), observing no differences in movement time between visual conditions, suggested that movement times should be insensitive to visual conditions since subjects will execute secondary submovements even in the absence of visual feedback.

The impact of the visual manipulation on movement trajectories was primarily evident during the latter phase of the movement. The absence of an influence of vision on the magnitude of peak velocity suggests that visual regulation occurred primarily during the decelerative phase of the movement (Carson et al, in press) Indeed, the visual manipulation proved to be a potent determinant of the relative time spent in decelerating the movement Additionally, the time between peak velocity and the end of

8 Alternatively, a positive relationship between movement time and the number of modifications may indicate that subjects slow their movements to make modifications 
the movement also predicted terminal accuracy when vision of the cursor was available. It is also during this phase in which we see a visually related difference in the number of movement adjustments. Specifically, there were more zero crossings and significant deviations in the acceleration profile of movements executed in the presence of visual feedback.

It appears that the performance advantage observed in the Vision-cursor condition was derived from a modificational process subserved by vision. The secondary accelerations and deviations in the acceleration trace, reflecting adjustments to the trajectory, apparently contributed to better accuracy and consistency. This implies that the presence of these modifications should be negatively related to terminal accuracy. Indeed, the negative relationship, albeit small, between the number of modifications and absolute error indicates that these adjustments to the movement were functional in preserving terminal accuracy.

In agreement with previous studies (e.g., Carson et al., in press; Elliott et al., 1991; Jeannerod, 1984; Meyer et al., 1988), adjustments to the movement trajectory were also evident even in the absence of vision. This finding conflicts with models which posit that corrective submovements are mediated by a visual comparison of the positions of the limb and target (e.g., Keele, 1968, 1981). 
The presence of these adjustments despite the absence of visual information of the limb position suggests that they may be based upon other sources of information, for example, kinesthetic feedback. ${ }^{9}$ Given that performance remains inferior to that when vision is available, kinesthetic feedback appears to be a relatively poor source of information upon which to base corrective adjustments. Furthermore, in the present task, kinesthetic feedback is dissociated from the visual information and therefore would be expected to provide less direct information regarding the position of the limb/cursor, compared to a situation in which a traditional pointing task is employed.

Although the performance differences between the visual conditions are comparable to what has been demonstrated previously (e.g., Elliott et al., 1991), the kinematic findings are in contrast with others (e.g., Carson et al., 1992; Elliott et al., 1991; Meyer et al., 1988). One of the primary differences is the presence of an influence of vision condition upon the number of corrections. Previous studies (Carson et al., 1992; Elliott et al., 1991; Meyer et al., 1988) have failed to show any differences in the number of secondary accelerations. The present study demonstrates a

9 Another source may be feedforward information. The presence of significant deviations prior to peak velocity in both visual conditions suggests that feedforward information may be the basis of these adjustments, given that there may not be sufficient time for feedback utilization. 
difference in the number of zero crossings and significant deviations as a function of vision condition (see also carson et al., in press). The discrepancy between these studies and the present may partly be a function of the type of task employed and the more detailed kinematic analyses performed here.

Although secondary accelerations have been found in the acceleration traces of pointing movements (e.g., Carlton, 1981; Carson et al., 1992, in press; Elliott et al., 1991), these corrections have generally been regarded as being nonfunctional (Carson, Goodman, Elliott, \& Chua, 1991; Carson et al., in press; Elliott et al., 1991). In these previous studies (e.g., Carson et al., in press; Elliott et al., 1991), the presence of a secondary acceleration failed to predict terminal accuracy. Even when vision was available, accuracy was unrelated to the number of zero crossings in the acceleration trace (Carson et al., in press; Elliott et al., 1991). This independence of zero crossings and pointing accuracy may be expected if some movements are reasonably accurate and require no corrections, or alternatively, visual regulation of the limb movement proceeds in a more continuous fashion (Elliott et al., 1991). In the present study, the negative relation between the number of modifications and absolute error suggests that the modifications observed were functional, at least to some degree (cf. Carson et al., 1991; 
in press; Elliott et al., 1991). Still, the negative relationship is small. Moreover, it is independent of vision condition. One might initially expect a stronger relation if these modifications served to maintain terminal accuracy. Furthermore, if modifications made with visual feedback are assumed to be more accurate (Meyer et al., 1988), then one might expect a stronger negative relation when vision is available. The present data, however, do not support this prediction.

There are some differences between the correlational analyses performed in the present study and those in previous studies (Carson et al., in press; Elliott et al., 1991) which should be noted. The primary discrepancy is that in the correlations involving the number of modifications, the present analyses used the total number of modifications per trial. This total was therefore comprised of zero crossings, significant deviations and reversals. In contrast, previous studies (Carson et al., in press; Elliott et al., 1991) used only the number of zero crossings. Carson et al. (in press) enumerated significant deviations in addition to zero crossings and found visual influences similar to the present study; however, in examining whether the presence of an adjustment predicted terminal accuracy, only the number of zero crossings was used. It cannot be determined therefore, whether a significant relationship between the number of 
adjustments and accuracy would have been found if the total number were used rather than just the number of zero crossings (cf. Carson et al, in press

Experiment 2

I have argued earlier that Meyer et al 's (1988 assumption that secondary submovements are prepared on the basis of visual feedback of the primary submovement endpoint is inconsistent with the model's zero-delay constraint It was suggested that if the preparation of the secondary submovement is mediated by visual feedback, important visual information must be derived from earlier stages of the movement Specifically, relevant visual information from the movement trajectory must be picked up prior to the end of the primary submovement It was expected, therefore, that visual feedback from the earlier stages of the movement trajectory prior to the endpoint of the initial submovement, would be important for movement accuracy Absence of visual feedback from the terminal portions of the trajectory assumed to encompass the primary submovement endpoint) would be expected to have less influence on accuracy (cf. Carlton, 1981 Experiment 2, therefore, was designed to investigate the effects of selectively excluding visual feedback during different stages of the movement trajectory Visual feedback was manipulated by either providing vision of the cursor (Vision-cursor), no vision of the cursor (No Vision-cursor), 
vision of the cursor only on the first half of the trajectory (FHV), or vision of the cursor only on the last half of the trajectory (LHV).

In experiments involving visual feedback manipulations, investigators are often concerned with the strategy adopted by subjects. If subjects were to adopt different strategies depending on the visual conditions, the results may be confounded. For example, when vision is provided only on a particular portion of the movement trajectory (e.g., see Carlton, 1981), subjects may decrease the speed of their movements when vision becomes available, thereby allowing them to utilize visual feedback. In this study, the goal was for subjects to maintain the same strategy across visual conditions. The intent was to have subjects prepare to move to targets in the same manner, regardless of whether complete or only partial feedback was available. It would not be too surprising to find that different strategies are employed for Full Vision and No Vision conditions (e.g., Elliott \& Allard, 1985; Zelaznik, Hawkins, \& Kisselburgh, 1983). Of greater concern was that different strategies were not adopted for the partial feedback conditions.

Therefore, the four visual conditions were not assigned to separate blocks of trials. In attempts to avoid the problem of varying movement strategies, the two partial 
feedback conditions were combined with either a Vision-cursor or No Vision-cursor condition. Thus, a block of trials consisted of either the vision-cursor condition combined with the two Partial-Vision conditions, or the No Vision-cursor condition combined with the partial-Vision conditions. It was of interest to see whether the results for the two partial feedback conditions would differ depending on whether they were combined with the Vision-cursor or No Vision-cursor condition.

Since varying target size had only a minimal influence on the dependent measures in Experiment 1, this factor was eliminated in Experiment 2. Thus, Vision condition remained the sole independent factor. Subjects in the present study also received more extensive practice than subjects in Experiment 1. Although practice effects were not dealt with directly in this work, it was an issue which could be examined afterward, should it prove to be of interest. Therefore, subjects in the present study participated in an additional experimental session identical to Experiment 1 . This session was considered as practice.

\section{Method}

Subjects

Subjects were 10 right-handed (Oldfield, 1971) undergraduate and graduate students $(5$ male, 5 female; age: 
17 - 28 years) from McMaster university. All had normal or corrected-to-normal vision.

Apparatus

The apparatus were identical to those in Experiment

1.

Procedure

There were four types of visual feedback

manipulations. These were: 1) Vision-cursor (VC), in which vision of the cursor was available throughout the movement, 2) No Vision-cursor (NC), in which the cursor was extinguished immediately upon leaving the home circle, 3) First-Half Vision (FHV), in which vision of the cursor was available only on the first half of the trajectory and was extinguished when the cursor reached the midpoint of the distance to the target, and 4) Last-Half Vision (LHV), in which the cursor was extinguished upon leaving the home circle and subsequently reappeared upon reaching the midpoint of the trajectory, thus providing vision of the cursor only after it crossed the midpoint. A single target size (10 mm) and distance $(130 \mathrm{~mm})$ was used, yielding an index of difficulty of 4.7 .

The four types of vision manipulations were combined to form two vision combination groups. These were the Vision Mix (VX), in which the two Partial-Vision manipulations, First-Half Vision and Last-Half Vision, were combined with 
the Vision-cursor condition, and the No Vision Mix (NX), in which the two Partial-Vision manipulations were combined with the No Vision-cursor condition. These two vision combination groups therefore yielded six unique vision conditions. These were 1) Vision-cursor (VC), 2) First-Half Vision within a Vision Mix (VXFH), 3) Last-Half Vision within a Vision mix (VXLH), 4) No Vision-cursor (NC), 5) First-Half Vision within a No Vision Mix (NXFH), and 6) Last-Half Vision within a No Vision $\operatorname{Mix}(\mathrm{NXLH})$

A one factor (Vision condition) repeated measures design was utilized. Each vision combination group was assigned to a block. Thus, each block was comprised of three of the vision conditions. Each block consisted of 100 trials which were divided into 60 trials of Vision-cursor for No Vision), 20 trials First-Half Vision, and 20 trials Last-Half Vision. Within each block, trials for the three vision conditions were randomized. Each subject participated in two testing sessions (60 minutes/session), generally held on successive days. The first experimental session was completely identical to Experiment 1. The second session was comprised of two practice blocks and the two vision combination blocks of Experiment 2. Vision combination/block order was counterbalanced across subjects. Prior to each vision combination block, a block of 20 practice trials was performed. If the subsequent block was a Vision Mix, 
subjects received a practice block of vision-cursor trials. If the subsequent block was a No Vision Mix, then subjects performed a practice block of No Vision-cursor trials. Prior to each vision combination block, subjects were told that the majority of the trials would be Vision-cursor (or No Vision) trials, and that on some trials, vision of the cursor would be limited to a portion of the trajectory. At the start of the Vision Mix block, subjects were instructed to prepare to use the available visual information and to prepare as they normally would in a block of vision-cursor trials. Similarly, in the No Vision Mix block, subjects were advised to prepare as they normally would during a block of No Vision-cursor trials.

Since it was also of interest to investigate whether performance might vary in the Partial-Vision conditions depending upon whether they were embedded within a Vision Mix or No Vision Mix or blocked on their own, two additional blocks of Partial-Vision trials were collected. Since the data from these trials were not included as part of the main analyses, these blocks were conducted at the end of the second session to avoid any influence on the results in the other conditions. One block of 20 First-Half Vision and one block of 20 Last-Half Vision trials were performed, the order of which was counterbalanced across subjects. 
The remainder of the procedures were identical to those in Experiment 1.

Data Reduction

The data from the first session were not analyzed. For the Vision Mix block, 4 Vision-cursor trials were picked at random from trials 1-20, 21-40, 41-60, 61-80, and 81-100, thus giving a total of 20 trials. The same process was performed for No Vision trials from the No Vision Mix block. These trials were analyzed along with the trials for the Partial-Vision conditions. Thus, there were 20 trials for each of the six vision conditions.

The remainder of the data reduction and kinematic analyses, and the dependent variables were identical to Experiment 1.

\section{Results}

Mean values were calculated for each of the dependent variables from the 20 trials in each vision condition. The dependent variables were analyzed separately using a one factor (Vision condition) repeated measures analysis of variance. Post-hoc examination of vision effects utilized the weighted means comparison procedure. This procedure is similar to planned comparisons, with the exception that contrasts need not be orthogonal to each other as a result of significance being first established with the one-way analysis of variance. Adopting this approach allowed for 
more meaningful comparisons than what multiple pairwise comparisons would yield. The four variables relating to movement modifications were analysed using Friedman analysis of variance by ranks due to marked skewness in the distributions.

\section{Performance Measures}

In contrast to Experiment 1, analysis of movement time revealed this variable to be independent of Vision condition. Although the majority of trials (60\%) within a vision combination group were either Vision-cursor or No Vision-cursor, the movement time effect found for these conditions in Experiment 1 was absent here. Perhaps, the presence of the Partial-Vision conditions (FHV and LHV) within the two Vision Mix blocks negated the movement time difference found previously.

Examination of constant error revealed an overall tendency to overshoot (grand mean $=0.81, \mathrm{p}<.01$ ) and an effect for Vision condition, $\underline{E}(5,45)=7.12, \underline{D}<.001$. Figure 15 illustrates the mean constant error of subjects in the six Vision conditions. Post-hoc analyses of these means revealed that the Vision-cursor condition was significantly different from a combination of the two First-Half Vision conditions (VXFH $+\mathrm{NXFH}), \underline{E}(1,45)=10.53, \underline{\mathrm{D}}<.01$. As depicted in Figure 15, subjects, on average, were accurate and exhibited essentially equal bias when vision of the 


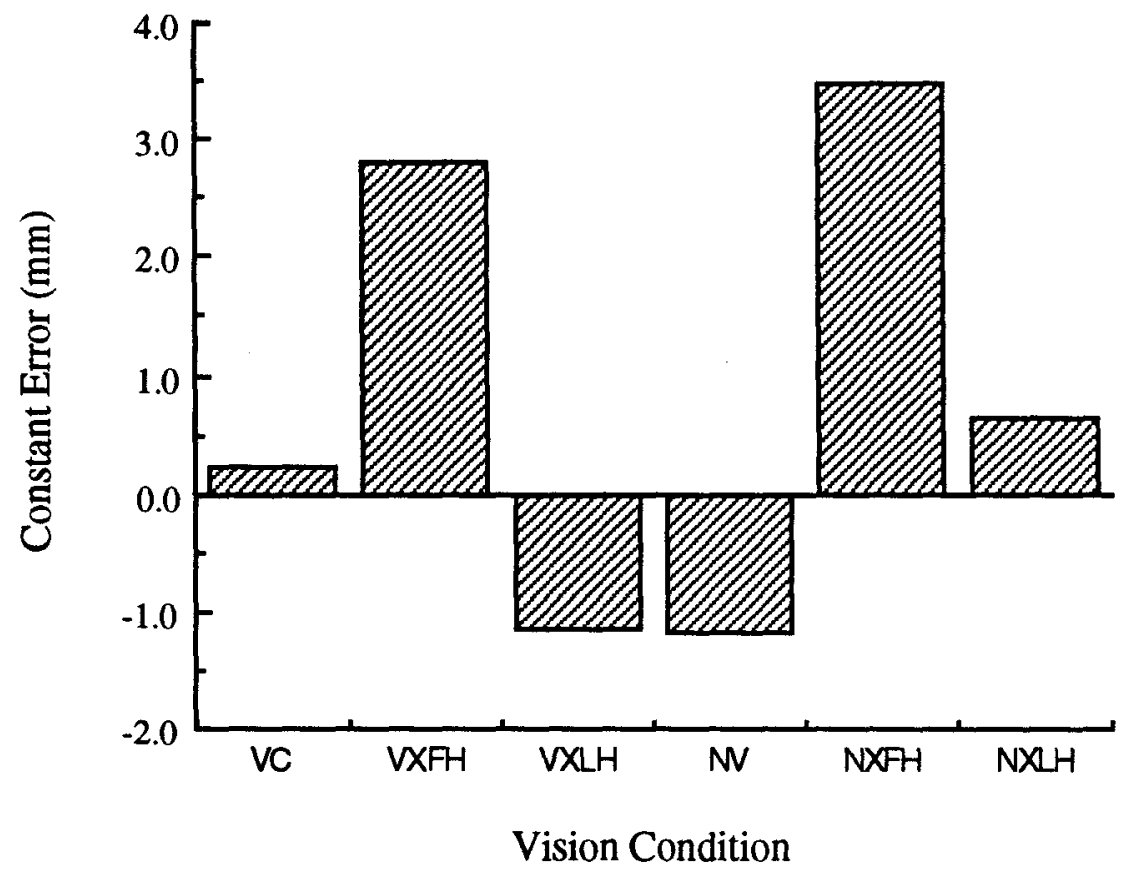

Figure 15. Experiment 2: Constant error (mm) as a function of Vision condition. 
cursor was available, but overshot the target center when vision was available only during the first half of the trajectory. A comparison of the two First-Half Vision conditions against the two Last-Half Vision conditions showed better performance by subjects in the latter conditions, $\underline{E}(1,45)=21.43, \underline{D}<001$ This is presumably a consequence of the longer duration for which visual information is available in the Last-Half Vision conditions and the proximity of the cursor to the target during this period No differences were found between these conditions and the Vision-cursor condition. Interestingly, no constant error differences were found between the Vision-cursor and No Vision conditions, in contrast to what might have been expected from previous findings (e g, Elliott et al, 1991; Prablanc et al., 1979a). There was a difference between the No Vision condition and the two First-Half Vision conditions, $\underline{F}(1,45)=2297, \underline{D}<001$

Performance differences were also evident in the analyses for variable error The availability of visual information was found to influence subjects' consistency, $\underline{E}(5,45)=967, \underline{D}<001$ Post-hoc comparisons examining specific questions revealed that subjects were less variable when they were certain that some visual information would be available during the ensuing movement compared to the situation in which such certainty was not available, $\underline{E} 1,45$ 
$=20.27, \mathrm{D}<.001$. Figure 16 illustrates these differences between the Vision-cursor combination conditions and the No Vision-cursor combination conditions. Further comparisons showed that vision of the cursor throughout the movement was of benefit compared to vision only on the first half (VXFH +

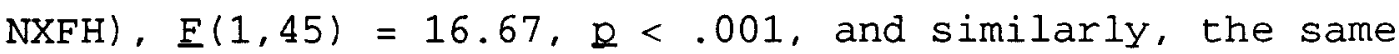
benefit was evident for vision during the last half, $E(1,45)$ $=15.93, \underline{\mathrm{D}}<.001,(\mathrm{VXLH}+\mathrm{NXLH}$ VS VXFH $+\mathrm{NXFH})$. As with constant error, there were no differences between the Visioncursor condition and the two Last-Half Vision conditions. Although performance in the First-Half Vision conditions were inferior to the Vision and Last-Half Vision conditions, it should be noted, however, that a comparison of the two FirstHalf Vision conditions against the No Vision condition revealed that some benefit was gained from the early visual information that was available, $\underline{E}(1,45)=4.71, \underline{p}<.05$. A final comparison to examine any possible differences between the Partial-Vision conditions in the Vision Mix and those in the No Vision Mix (VXFH + VXLH VS NXFH + NXLH) revealed no significant differences. Thus, performance variability in the Partial-Vision conditions were independent of the type of vision combination group (Vision vs No Vision) in which these partial conditions were embedded.

\section{Kinematic Measures}

Analysis of peak velocity revealed that, similar to 


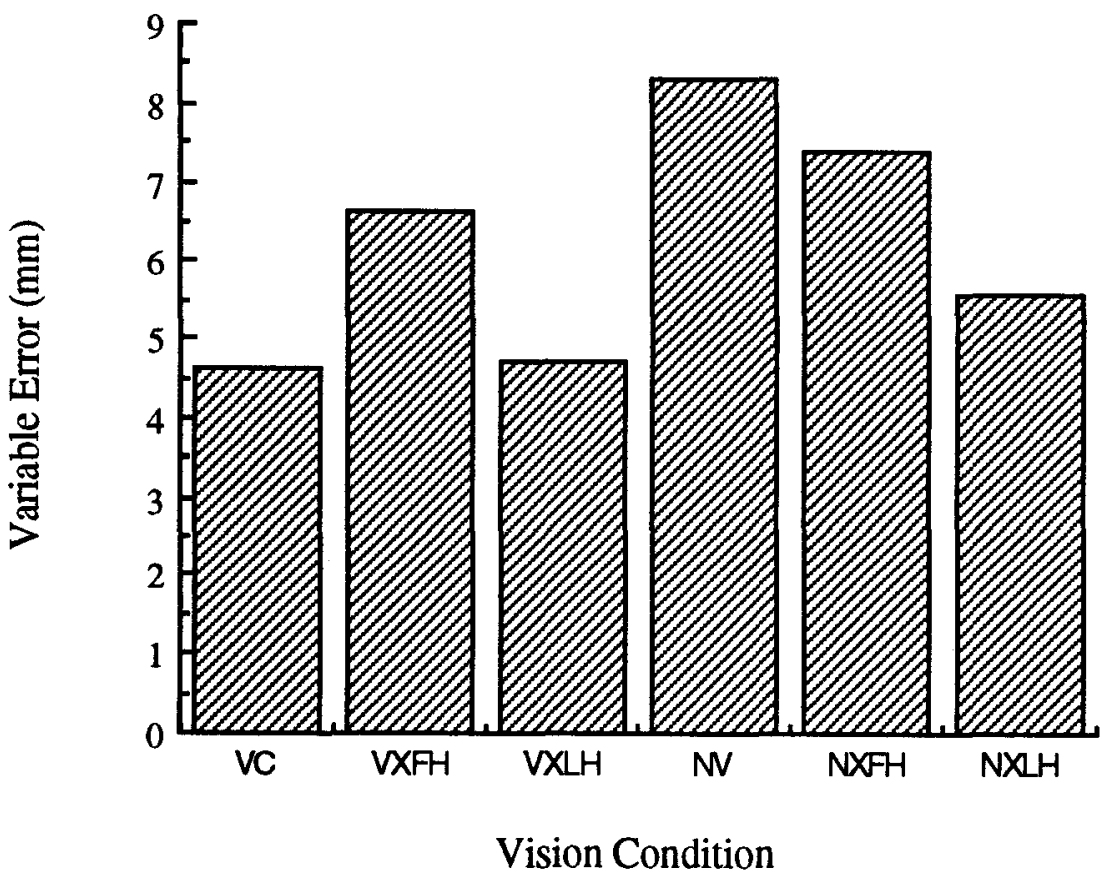

Figure 16. Experiment 2: Variable error (mm) as a function of Vision condition. 
Experiment 1, this kinematic variable was independent of Vision condition (e.g., Carson et al., in press; Elliott et al., 1991). In contrast, an effect for Vision condition was found for both the time to peak velocity as a proportion of movement time $(\mathrm{TPV} / \mathrm{MT}), \underline{F}(5,45)=3.62, \mathrm{p}<.01$, and the proportion of the target distance travelled at peak velocity (PVDISP/DIST), $\mathbf{E}(5,45)=4.25, \mathrm{D}<.005$. An examination of these effects revealed that for both variables, the primary influence was exerted by the type of vision combination group

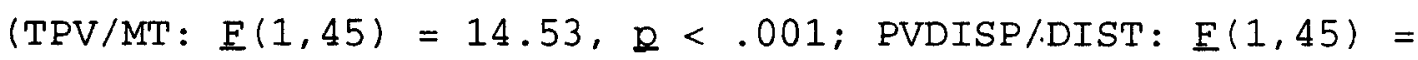
$21.12, \mathrm{D}<.001)$. Subjects spent a greater proportion of the movement in the deceleration phase when they were certain that the cursor would always be visible for at least a portion of the trajectory (see Figure 17). Moreover, as a likely consequence of attaining peak velocity earlier proportionally in vision situations, subjects also travelled a lesser proportion of the trajectory at the time of peak velocity (see Figure 18). These effects were similar to Experiment 1, although the differences observed in the present experiment were smaller. Note that in all conditions, subjects were already decelerating their movements when the cursor either appeared (LHV condition) or disappeared (FHV condition) at the midpoint of the trajectory.

Analyses of movement modifications employing Friedman 


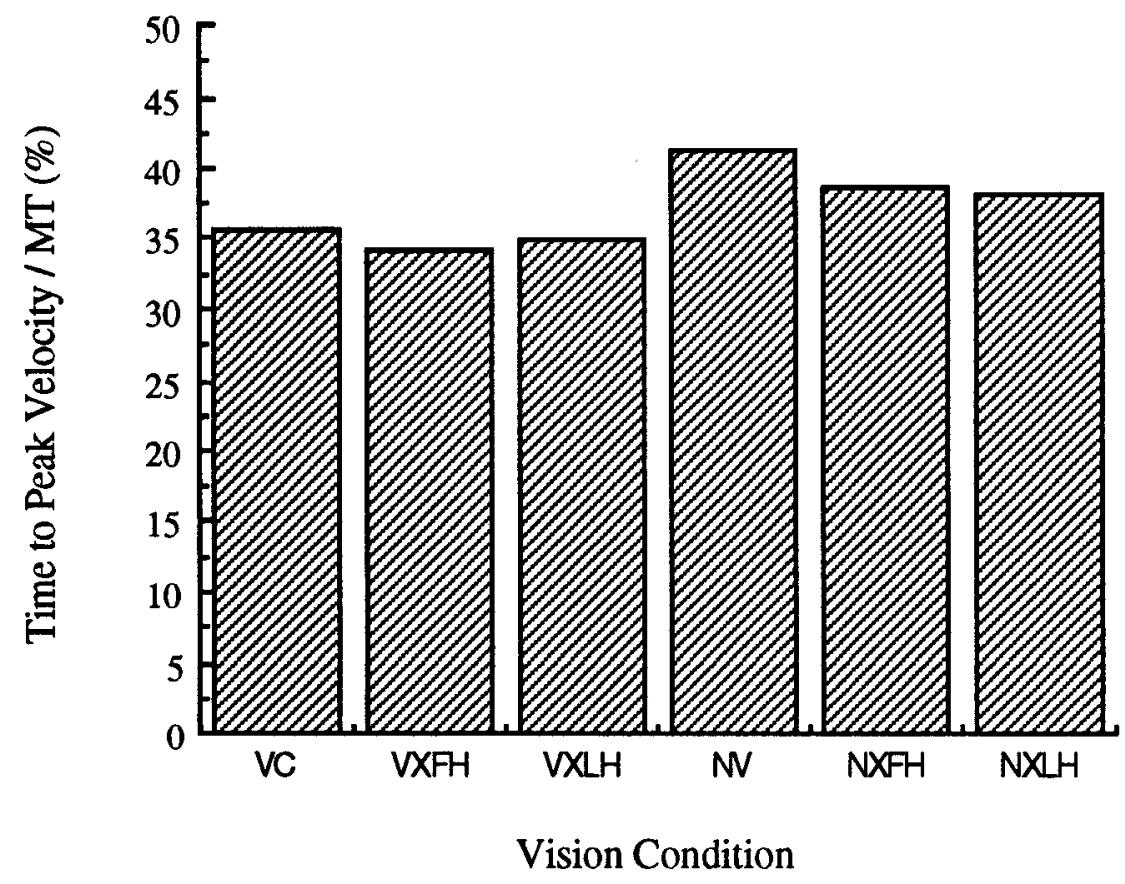

Figure 17. Experiment 2: Time to peak velocity, expressed as a proportion of movement time (\%), as a function of Vision condition. 


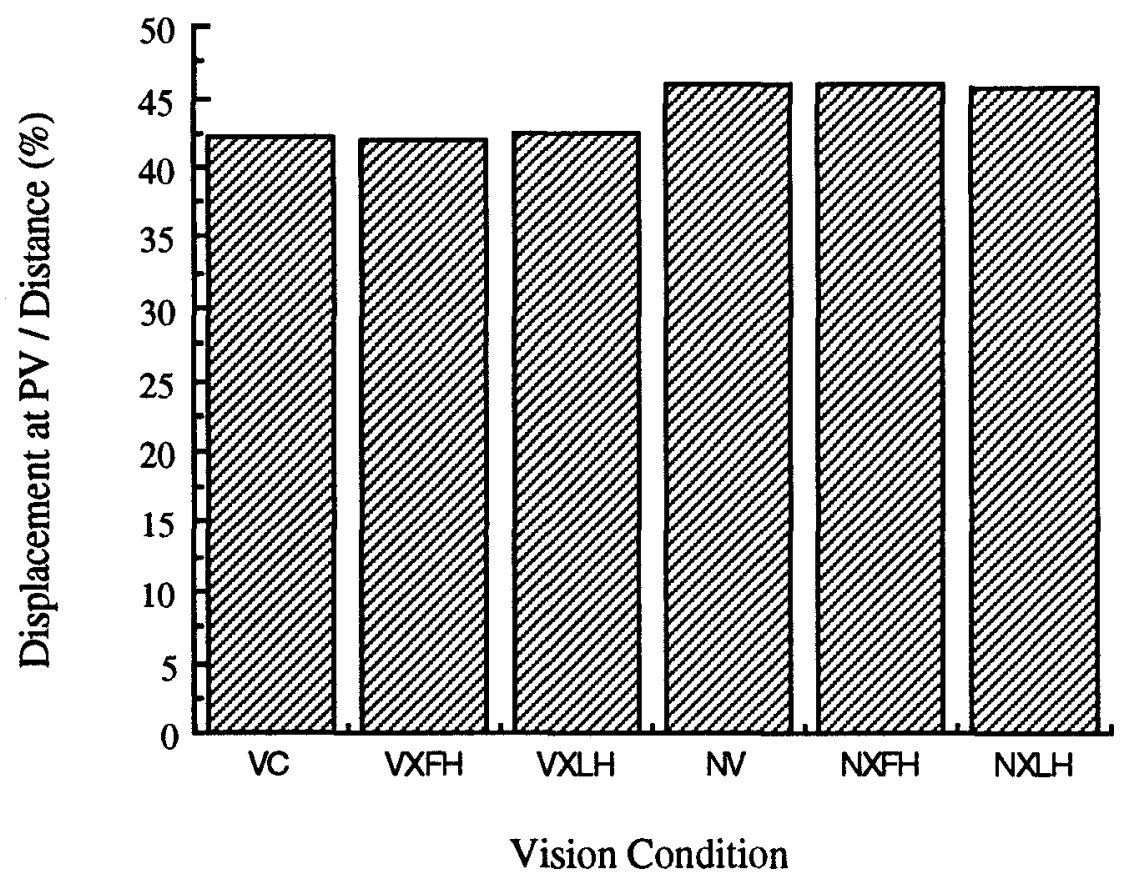

Figure 18. Experiment 2: Displacement at time of peak velocity, expressed as a proportion of target distance (\%), as a function Vision condition. 
analysis of variance failed to reveal a significant effect for any of the four modification variables (see Appendix C, Table 26). The absence of an effect for vision condition is consistent with the results of previous investigators (Elliott et al., 1991; Meyer et al., 1988) but is in direct contrast with those of Experiment 1. The situation arises, therefore, in which similar performance advantages are found for movements made under visual guidance, with the notable exception that, unlike Experiment 1, these advantages which are presumably due to the presence of visual information are no longer related to the number of movement modifications. 10 Correlational Analyses

Correlational analyses identical to Experiment 1 were performed on the same group of dependent variables.

There was an overall positive relation between MT and MODS (grand mean $z=0.76, \underline{Q}<.001$ ). An influence of vision condition, $\underline{E}(5,45)=3.02, \underline{p}<.05$, was due to a significant pairwise difference between the two extreme means (Tukey HSD, Q $<.05)$.

A slight negative relation was found between TPV-END and $\mathrm{AE}$ (grand mean $\mathrm{z}=-0.19, \mathrm{Q}<.01$ ). Once again, post-hoc analysis (Tukey HSD, $\underline{D}<.05$ ) of an effect for vision

10 A one-way repeated measures ANOVA performed on the total number of modifications also failed to reveal any influence of vision condition. 
condition, $\underline{E}(5,45)=3.83, \underline{P}<.01$, revealed only a difference between the extreme means.

There was also a small negative relation between MODS and $\mathrm{AE}$ (grand mean $\mathrm{z}=-0.14, \mathrm{D}<.01$ ). A significant difference between the two extreme means (Tukey HSD, $\mathbb{P}<.05$ ) resulted in an effect for vision condition, $\underline{F}(5,45)=3.12, \mathbb{D}$ $<.05$.

\section{Partial-Vision conditions}

To examine whether the manner in which the PartialVision conditions were grouped (ie., in combination with Vision trials, or with No Vision trials, or blocked on their own) affected performance and the manner in which subjects prepared for upcoming movements, analyses were performed which included the data from the blocked Partial-Vision conditions. The analyses utilized a factorial arrangement of two Partial-Vision conditions (FHV and LHV) and three Mixtype conditions (Partial-Vision conditions in combination with Vision-cursor trials (VX), in combination with No Vision trials (NX), and blocked on their own (BLK)), to employ a 2 x 3 (Partial-Vision $\mathrm{x}$ Mix-type) repeated measures analysis of variance.

Performance Measures. Analysis of movement time revealed an effect for Partial-Vision condition, $\underline{F}(1,9)=$ $5.74, \mathrm{p}<.05$, with subjects taking longer to complete their movements in the last-half Vision conditions (FHV $=593 \mathrm{~ms}$; 
$\mathrm{LHV}=640 \mathrm{~ms})$. Presumably, the longer movement times reflect the subjects' attempts to utilize the visual information which becomes available once the midpoint of the trajectory is reached and to make any necessary adjustments to the movement.

Subjects' movement accuracy and bias, reflected in constant error, was affected by both Partial-Vision condition, $\underline{E}(1,9)=32.13, \underline{D}<.001$, and Mix-type, $\underline{E}(2,18)=$ $3.83, \underline{\mathrm{D}}<.05$. As might be expected, subjects were more accurate when visual information was available on the last half of the trajectory $(-0.28 \mathrm{~mm})$. In contrast, when visual information was restricted to the initial half of the trajectory, subjects overshot the target center $(2.54 \mathrm{~mm})$. Figure 19 illustrates the overall overshooting that occurred in the First-Half Vision conditions. The reason for this remains unclear at this point. When the Partial-Vision trials were either blocked or combined with vision-cursor trials, subjects were more accurate (Vision $\mathrm{Mix}=0.84$; Blocked $=0.49$ ) than the situation in which these trials were combined with No Vision trials (No Vision Mix $=2.06$ ). A significant pairwise difference was found between the Blocked and No Vision Mix conditions (Tukey HSD, $\mathbb{Q}<.05$ ). It seems subjects tended to be more accurate when certain that some visual information would be available during the ensuing 


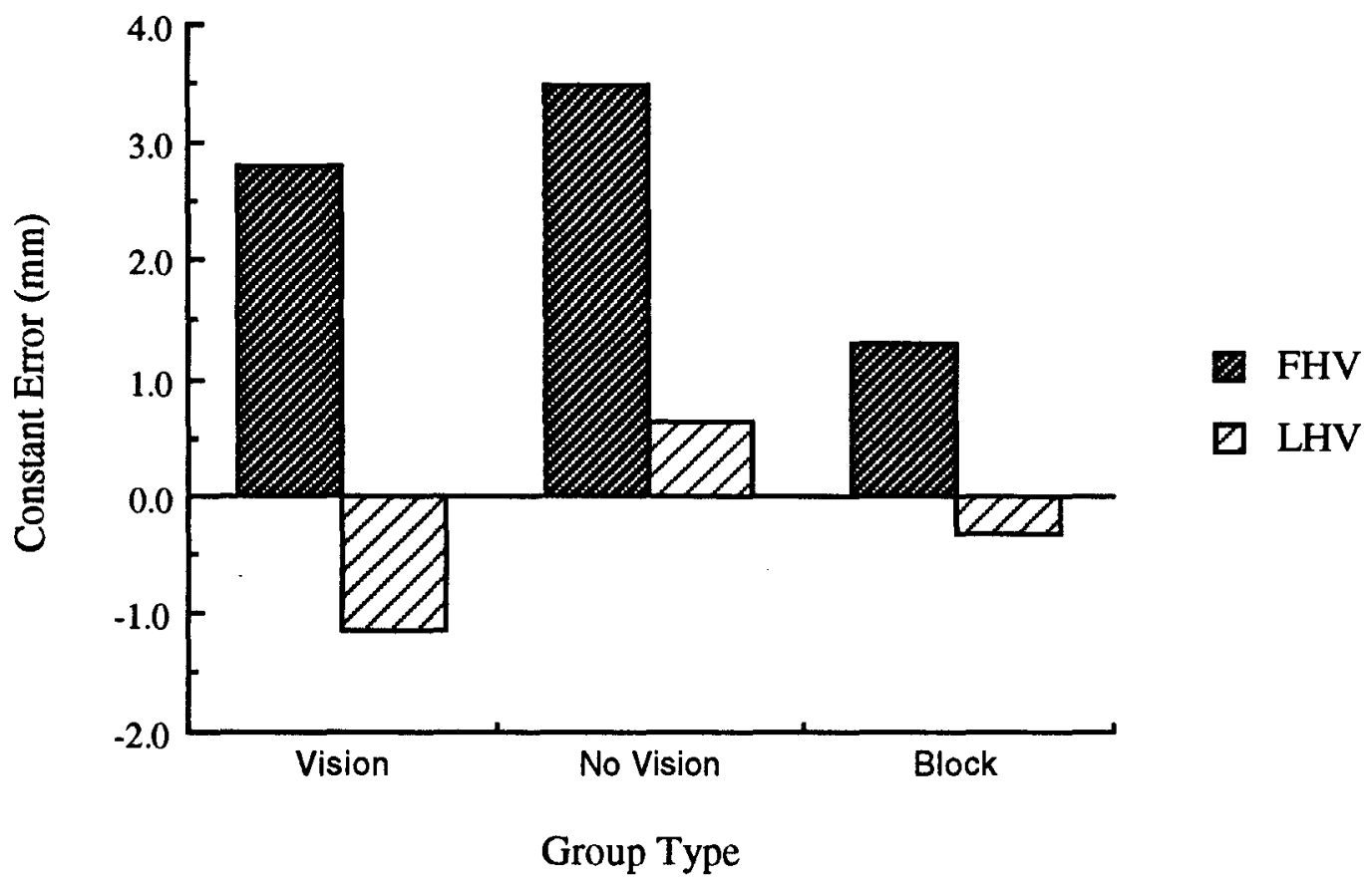

Figure 19. Experiment 2: Constant error (mm) as a function of Partial-Vision condition and Group Type. 
movement (e.g., see Elliott \& Allard, 1985; Zelaznik et al., 1983).

Partial-Vision condition was the sole influence on variable error, $\underline{E}(1,9)=26.70, \underline{\underline{D}}<.001$. Once again, with the longer durations for which visual information is available during the Last-Half Vision conditions, subjects were able to maintain greater consistency $(\mathrm{FHV}=7.10 \mathrm{~mm}$; LHV $=5.11$ ) compared to the First-Half Vision conditions.

Kinematic Measures. Analysis of peak velocity again revealed no significant effects, while the analyses for TPV/MT and PVDISP/DIST revealed only an interaction of Partial-Vision and Mix-type conditions for both variables $(\mathrm{TPV} / \mathrm{MT}: \underline{\mathrm{E}}(2,18)=5.36, \underline{\mathrm{D}}<.025 ;$ PVDISP/DIST $: \underline{\mathrm{F}}(2,18)=$ $10.487, \underline{D}=.001$. As is evident in Figure 20, the interaction for TPV/MT was reflective of subjects spending a greater proportion of the movement in deceleration in the Last-Half Vision condition compared to the First-Half Vision condition when these conditions were blocked. When these conditions were combined with either Vision or No Vision trials, there was no such differences. In parallel, subjects travelled a lesser proportion of the trajectory in the LastHalf condition compared to the First-Half condition when these conditions were blocked, whereas no difference was found when these conditions were embedded within the other two Mix-type conditions (see Figure 21). 


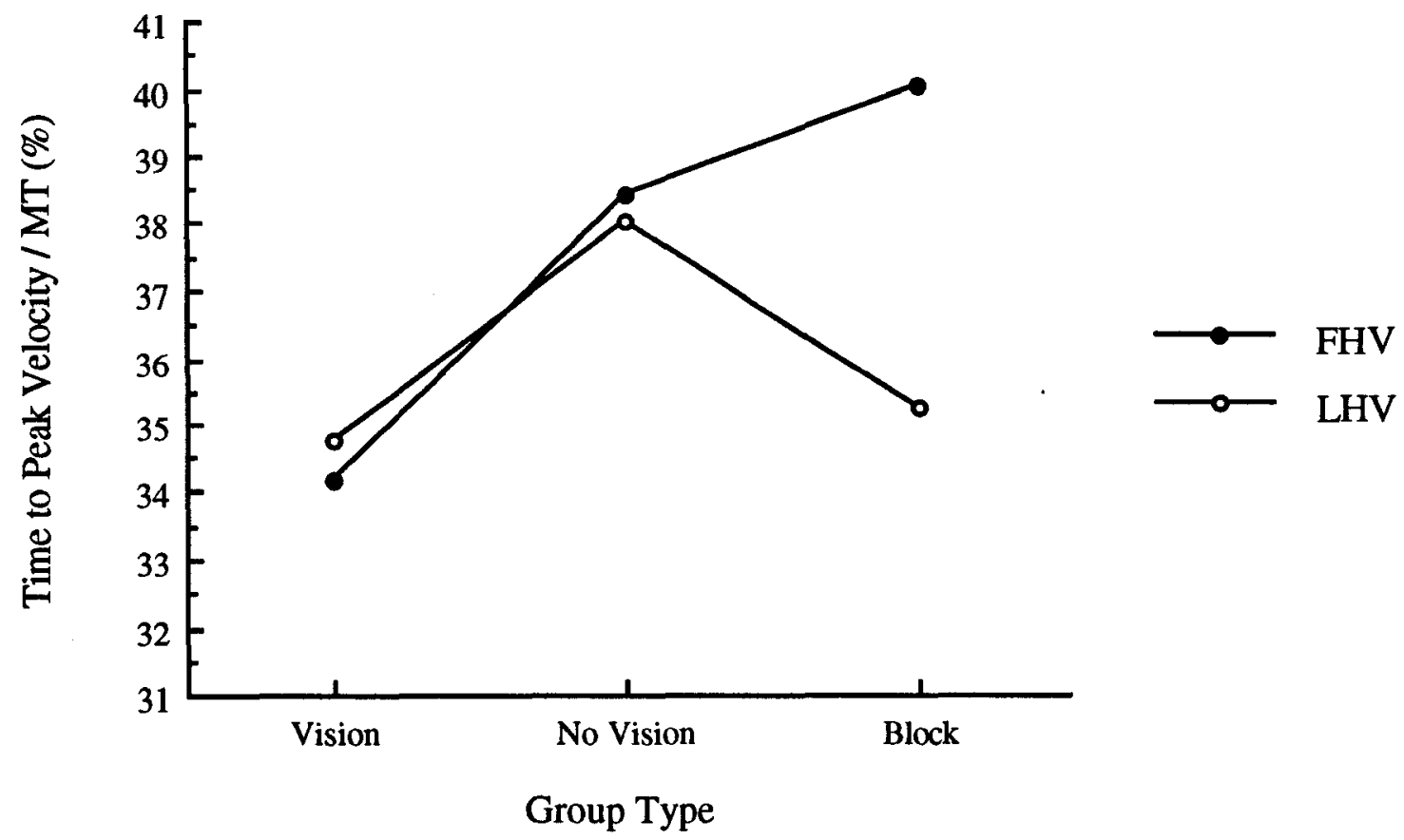

Eigure 20. Experiment 2: Time to peak velocity, expressed as a proportion of movement time $(\%)$, as a function of PartialVision condition and Group Type (mm). 


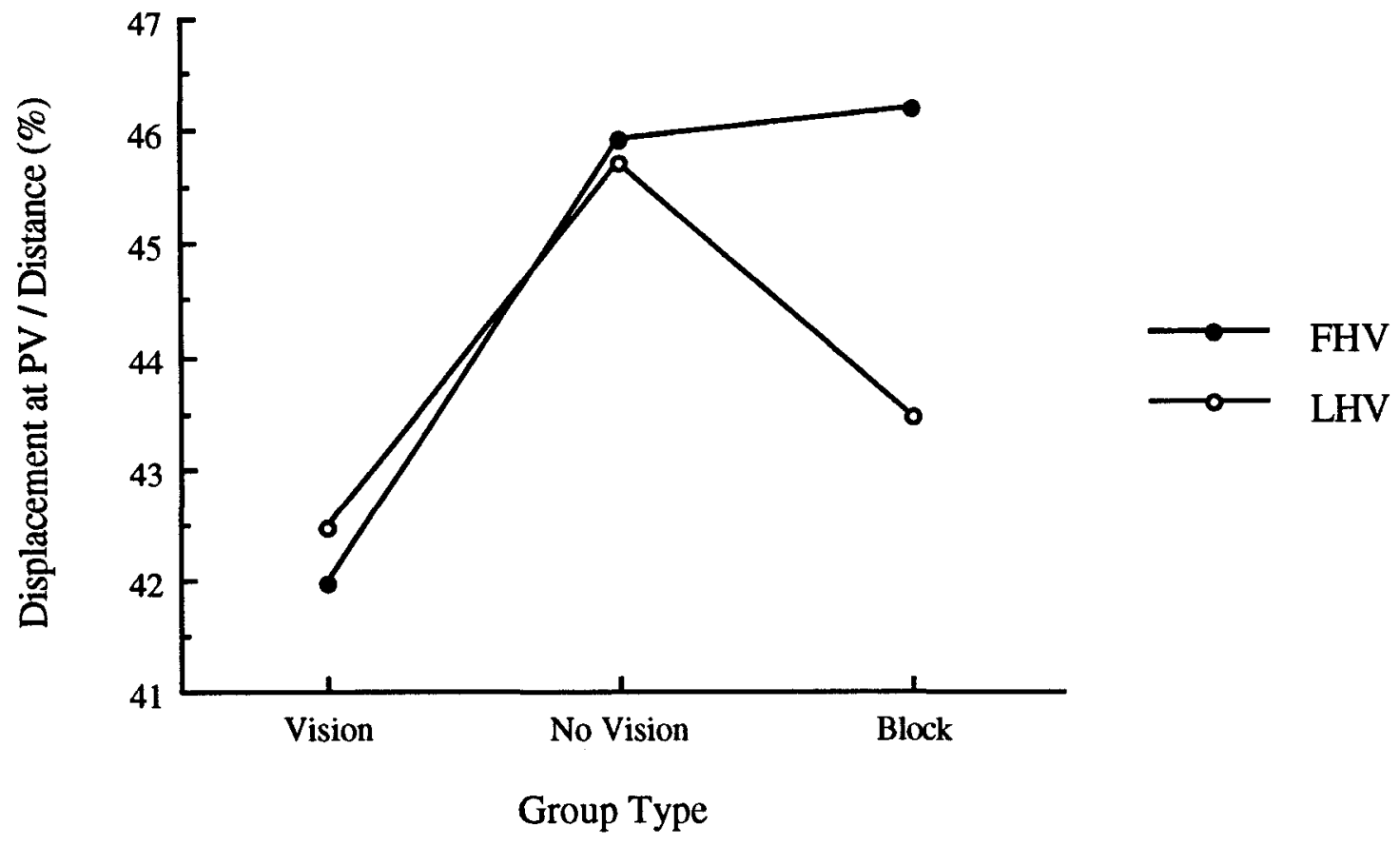

Figure 21. Experiment 2: Displacement at time of peak velocity, expressed as a proportion of target distance (\%), as a function of Partial-Vision condition and Group Type (mm). 
Initial six level Friedman anovas performed on each of the variables relating to movement modifications once again revealed no significant effects for any one of the variables. Therefore, in parallel to the findings in the main analyses, the performance advantages found for the LastHalf Vision conditions are apparently unrelated to the number of modifications made to the movement trajectory.

\section{Discussion}

The primary purpose of this study was to examine the effects of selectively excluding visual feedback on aiming performance and dynamics. If corrective adjustments are based on visual feedback from the preceding initial submovement, then, given the intrinsic delays in visuomotor processing, this feedback must be derived from the earlier stages of the initial submovement and not its endpoint (cf. Meyer et al., 1988). This implies, with respect to the present study, that important visual information can be gained from the first half of the movement trajectory (cf. Carlton, 1981).

In contrast to Experiment 1, constant error was no longer independent of vision condition, nor were the endpoints of the movements distributed about the center of the target. Both of these findings contrast with the predictions of the dual submovement model (Meyer et al., 1988). Subjects exhibited an overall tendency to overshoot 
the center of the target. This finding is in opposition to aiming studies (e.g., Elliott \& Allard, 1985; Jeannerod, 1988; Prablanc et al., 1979a, 1979b, 1986) in which the usual tendency is to undershoot the target. Interestingly, the overshooting was evident primarily when vision of the cursor was available only on the first half of the trajectory. Why this particular visual condition should affect pointing bias in this manner is not immediately obvious. In terms of constant error, receiving vision on the first half of the trajectory was actually detrimental when compared to the situation in which no visual feedback was provided. Clearly, there must be some underlying process which can account for this consistent bias in aiming during First-Half vision trials. The difference between this visual condition and the No Vision condition suggests that subjects probably did attempt to utilize the visual information from the first half of the trajectory. If subjects tried to use this early visual information to derive the speed of the cursor and their limb movement ${ }^{11}$, then perhaps, they may have consistently underestimated the speed and may therefore have applied less decelerative force, consequently terminating their movement beyond the target center. Alternatively, rather than underestimating the speed of the cursor/limb,

11 .Subjects' comments indicated that they attempted to utilize the early visual information to gain information about the speed of the cursor. 
perhaps subjects underestimated the precise location at which the cursor was extinguished, leading to the perception that a greater distance to the target remained.

The findings for variable error generally paralleled what might have been expected. Subjects were more consistent when vision was available throughout the movement or on the last half of the trajectory than when vision was available only on the first half or was not provided. In contrast to the findings for constant error, receiving visual feedback on the first half of the trajectory was of some benefit compared to not receiving any feedback. Thus, in conjunction with Experiment 1, there is evidence of an advantage derived from the availability of visual information.

Movement time differences between the visual conditions were no longer apparent. Embedding the PartialVision conditions within the blocks of Vision-cursor and No Vision trials apparently negated the movement time differences found previously (see Experiment 1). The secondary Partial-Vision analysis, however, does reveal that movements were generally of longer duration when vision was available on the latter half of the trajectory compared to the initial half. This suggests that during the Last-Half vision trials, subjects slowed their movements when vision of the cursor became available. Presumably, this strategy was 
adopted in order to utilize the available visual information and effect any necessary modifications.

It appears that movement regulation based on visual feedback occurred primarily during the latter phase of the movement. In support of this, the magnitude of peak velocity was insensitive to the visual manipulation, and furthermore, subjects once again spent a greater proportion of their movement time decelerating their movements. In Experiment 1, a longer decelerative phase was associated with better terminal accuracy when vision was available. Moreover, the visually-based performance advantage was attributed to a greater number of visual feedback-based adjustments to the movement. In the present study, the time between peak velocity and the end of the movement again predicted absolute error. However, the relationship was small and there was a negligible effect of vision. A difference of greater importance is that the present performance advantage can no longer be attributed to a prevalence of visually-based corrective adjustments. The absence of a visual influence on the number of modifications in the trajectory is in direct opposition to Experiment 1 (see also Carson et al., in press), but is consistent with other work (e.g., Carson et al., 1992; Elliott et al., 1991; Meyer et al., 1988). In summary, receiving visual feedback during the movement generally benefitted terminal accuracy and 
consistency. However, whereas there was evidence in the previous experiment to suggest that the superior performance may be attributable to the presence of modifications in the trajectory, the present findings indicate otherwise. One additional point of interest concerns the performance differences between the vision conditions. I initially expected that subjects would benefit from early visual feedback to a greater extent than what was found. However, the present findings indicate that greater advantages were accrued from visual information on the latter half of the movement. There are two primary issues which therefore merit further consideration. First, what accounts for the performance superiority with vision, given that the present kinematic indices no longer reflect the advantage? Second, why were there only limited benefits from visual information on the initial half of the trajectory? These questions shall be considered in turn.

There are a number of possibilities why the visually mediated performance advantage was no longer reflected in the kinematic measures. First, there were a greater number of errors (target missed completely) committed in the Visioncursor trials compared to Experiment 1. Those subjects who committed a greater number of errors may have paid insufficient attention to accuracy demands. It may be that had these subjects complied with accuracy demands, a greater 
number of corrections would have been evident in the Visioncursor trials. The assumption is, of course, that these adjustments are required and lead to better terminal accuracy (e.g., see Experiment 1). However, despite the larger number of errors committed, the impact of vision upon aiming consistency still remains evident. A second alternative is that the more extensive practice received by the subjects (cf. Experiment 1) may have resulted in a mode of visualmotor control to which the current kinematic indices were insensitive. This issue merits further investigation beyond the scope of the present work. On a related note, a third possibility is that visual regulation of the aiming movements occurred in a continuous, as well as a discrete, fashion (e.g., see Carson et al., 1992, in press; Elliott et al., 1991). Although the use of significant deviations in the acceleration trace was originally proposed to index a continuous or pseudocontinuous mode of visuomotor control, the present evidence suggests that this measure was not sufficiently sensitive to the modificational process that must have occurred when visual feedback was available.

The impact of excluding visual feedback from the latter half of the trajectory was greater than that of excluding feedback from the initial half. These results are supportive of the work of Carlton (1981), in that vision of the latter stages of the trajectory seems more critical for 
performance. However, there is evidence to suggest that early visual information was beneficial when compared to a No Vision condition (cf. Carlton, 1981; Beaubaton \& Hay, 1986). I initially expected that this early visual information would have a greater contribution. There are two possibilities which may explain the limited benefits to performance.

First, the term Last-Half vision (and First-Half vision) may be somewhat of a misnomer. It refers strictly to the availability of visual information once the limb/cursor passes the midpoint of the distance toward the target. There is no reference to the actual amount of time spent within that particular portion of the trajectory. One possible problem with the present methodology is that in situations in which visual information is excluded on the initial portion of the trajectory, subjects may adopt a strategy in which they slow their movements once vision becomes available (e.g., see also Carlton, 1981). They may also spend a greater amount of time traversing this latter portion of the trajectory, thereby allowing sufficient time to utilize the visual information which becomes available. This may therefore compensate for the lack of visual information during the initial portion of the trajectory. In the present study, subjects were already beginning the decelerative phase of their movements when vision became available during the Last-Half vision trials. Movement times were also longer for 
Last-Half vision trials compared to those for First-Half vision trials. Presumably, the longer movement times are primarily reflective of a greater amount of time spent in the latter half of the trajectory during Last-Half vision trials. The end result of this is that the actual durations of visual feedback during the First-Half vision trials and Last-Half vision trials were not equivalent across the two visual conditions. Subjects may therefore have received visual feedback for a longer duration during the Last-Half vision trials, and perhaps, in conjunction with the greater proximity of the cursor at a time when a visual feedbackbased correction could be made (e.g., Carlton, 1979; Howarth et al., 1971), this resulted in better terminal accuracy and consistency. In a future experiment, one might consider constraining movement times across these visual conditions. This would not prevent differences in the proportion of time spent within different portions of the trajectory, but that in itself may be of interest.

A second possibility which may account for the limited benefits of early visual information in the present study concerns the nature of the task. During First-Half vision trials, if subjects focus their gaze on the target, the initial half of the trajectory would generally be in the 
lower periphery of the visual field.12 Thus, subjects receive early visual feedback of the cursor through peripheral vision. Bard, Hay, and Fleury (1985) have suggested that early visual information attained through peripheral vision is more important for detecting errors in the direction of aiming. In the present task, the aiming movement was along a single dimension and only errors in amplitude were of concern. Elliott and Allard (1985) have also shown that early visual information is useful if its importance for accurate performance is emphasized. Employing a traditional aiming task, Elliott and Allard (1985) induced large errors in aiming direction by having subjects wear goggles fitted with a laterally displacing prism. This made errors in aiming obvious to the subject and also increased the importance of early visual feedback information. Therefore, in the present study, the unidimensionality of the aiming task and the lesser importance of the early visual information (compared to when the cursor is close to the target and errors are easier to detect) may have reduced the impact of visual feedback from the initial half of the trajectory.

The original motive for selectively excluding visual feedback during the initial and latter halves of the movement

12 At the conclusion of the experiment, subjects indicated that they focused their attention primarily on the upper part of the monitor about the target. 
trajectory was not simply to examine the importance of early and late visual feedback (e.g., see also carlton, 1981). The plan was also to evaluate the prediction of the dual submovement model (Meyer et al., 1988) with respect to the use of visual feedback in preparing the secondary submovement. I have argued previously that if the preparation of this secondary submovement is based on visual feedback from the initial submovement, then this feedback must be derived prior to the endpoint of the primary submovement, and not the actual endpoint (e.g., Carson et al., in press; cf. Meyer et al., 1988). Consequently, visual feedback from the initial portion of the trajectory was thought to be important. In the present study, I assumed that the initial half of the trajectory would encompass that portion of the primary submovement prior to its endpoint, and the latter half of the trajectory would therefore contain the actual endpoint. Following this line of reasoning, I therefore hypothesized that the visual feedback from the initial half of the trajectory would be important, given that it encompassed that portion of the primary submovement from which visual information was required for the preparation of the secondary submovement (cf. Meyer et al., 1988). In retrospect however, these assumptions are not without problems, and the present study has not provided an adequate test of the proposed hypothesis. The clarification of the 
basis of the preparation of the corrective submovement might have implications for issues concerning the utilization of visual feedback for movement regulation. These problems are therefore discussed in greater detail.

First, assuming that a corrective submovement or adjustment is based on visual feedback, then in a situation in which a secondary submovement follows an initial submovement without delay, can the feedback be based on vision of the primary submovement endpoint? The apparent delays in visuomotor processing (e.g., see Jeannerod, 1988, for review) suggests that it cannot be. Therefore, important visual information must be accrued prior to the end of the primary submovement. Perhaps this information may then be used to extrapolate the endpoint of the primary submovement (e.g., Jeannerod \& Prablanc, 1983).

To examine this issue, let us first consider an

ideal, hypothetical, situation. Assume that a goal-directed pointing movement is composed only of an initial submovement and a secondary correction. One method of testing the above hypothesis would be to manipulate the availability of visual feedback of the endpoint of the primary submovement. One problem with this concerns how one would detect the endpoint and simultaneously eliminate visual feedback. For the moment however, assume that a primary submovement always ends at a specific point of the trajectory. What one might then do is 
to manipulate the availability of vision about this point of the trajectory (cf First-Half and Last-Half vision) The segment of the trajectory prior to this point is then assumed to contain the primary submovement trajectory excluding its endpoint, and the segment consequent to this point is assumed to contain the actual endpoint The prediction would then be that visual feedback during the initial segment would be important if a visually-based corrective submovement is to follow without delay. It would also be predicted that the absence of visual feedback from the latter segment would not affect accuracy If an attempt is made to utilize feedback of the endpoint, then some delay must be evident between the end of the initial and the beginning of the secondary submovement, presumably due to delays in visuomotor processing

Since the location of the endpoint of primary submovements probably vary from trial to trial, then the above paradigm would be inadequate for investigation of the problem However, the premise of the approach would be similar Clearly, one of the shortcomings of the present study is the simple manipulation of vision based on particular segments of the trajectory It is an imprecise manipulation with which to examine the present issues of interest An additional problem is that movements can contain several corrections, not just one This amplifies 
the problem at hand since now one must consider how later corrections are prepared. Therefore, the issue of the role of vision in the preparation of corrective submovements warrants further investigation.

The purpose of embedding the Partial-Vision trials within Vision and No Vision blocks was to attempt to prevent differences in movement strategy between the two PartialVision conditions. However, as discussed earlier, the attempt was apparently not successful. It was also of interest to examine whether any performance differences between the Partial-Vision, conditions would be evident depending on whether they were combined with Vision or No Vision trials. No such differences were found. Even when these trials were compared to the situation in which they were performed in a blocked fashion, the differences were negligible. The effects of embedding the Partial-Vision conditions within Vision and No Vision blocks generally seem to be to reduce the Vision-No Vision differences found in Experiment 1. Although subjects were instructed to prepare either a "Vision" movement or "No Vision" movement, the randomization of these trials along with Partial-Vision trials may have resulted in a somewhat different strategy. The reduction of visual effects, possibly as a result of the embedding and randomization procedure, is reminiscent of previous work (e.g., see Elliott \& Allard, 1985; Zelaznik et 
al., 1983). Perhaps the combined 40 percent of PartialVision trials randomized within a Vision or No Vision block was too high a percentage for movement strategies to remain unchanged from those in a blocked arrangement and for. examining any differences between the Partial-Vision conditions as a function of the type of trials with which they are randomized.

General Discussion

Since the pioneering work of Woodworth (1899), researchers in motor control have been interested in issues concerning the speed-accuracy tradeoff and the role of vision in the guidance of goal-directed movements. Closed-loop models of visuomotor control (e.g., Carlton, 1981; Crossman \& Goodeve, 1963/1983; Keele, 1968) have generally emphasized the importance of vision in mediating the modificational processes inherent in accurate limb movements. The precise nature of this regulatory process remains a topic of debate. The question: Does visual regulation proceed in an intermittent, discrete, fashion, or is the process continuous/pseudocontinuous? The present work sought to examine this issue. In addition, a recent model of limb control, the Stochastic Optimized Dual Submovement Model, proposed by Meyer et al. (1988), was evaluated. of primary interest was the role of visual feedback within the general 
framework of the model and specifically in the preparation of secondary, corrective, submovements.

A number of assumptions of the dual submovement model (Meyer et al., 1988) were tested in the present work. In Experiment 2, evidence was presented to refute the model's assumptions concerning the distribution of movement endpoints. In addition to the demonstration of an overall unequal bias, endpoint distribution also proved to be sensitive to visual conditions. In Experiment 1, results for movement time also contradicted the predictions of the dual submovement model. The model predicts that due to an equal number of secondary submovements being executed across varying visual conditions, movement durations should be independent of visual conditions. However, in Experiment 1, movement times were shorter in the absence of visual feedback, a phenomenon attributed to the lesser number of visually-based, time-consuming modifications in the movement trajectories.

The dual submovement model is based upon earlier work by Meyer and his colleagues (Meyer et al., 1982) in which the authors propose a model which assumes symmetry in the velocity and acceleration profiles. In the present work, these symmetry assumptions were not supported. Movement trajectories were generally characterized by a longer decelerative than accelerative phase (e.g., see also 
Mackenzie et al., 1987; Todor \& Smiley, 1985; Zelaznik et al., 1986). Moreover, the asymmetrical characteristics of these trajectories were influenced by visual conditions. One of the most striking differences between the present data and the dual submovement model involves one of the model's fundamental assumptions: the number of submovements. The present work demonstrated that movements may be composed of more than two submovements. There was a prevalence of movement trajectories which were characterized by several corrections. Moreover, there is evidence to suggest that visual conditions may affect the presence of these corrections. Of course, one question which should be considered is: what precisely is the definition of a submovement? Do the types of modifications enumerated here all qualify as submovements?

On a related note, the dual submovement model predicts that in situations in which the primary submovement misses the target, the proportion of primary submovements which fall short of the target (undershoot) should be equal to those which fall beyond the target (overshoot). This implies, with respect to the nature of the ensuing secondary submovements, that there should be an equal proportion of secondary submovements which are reversals back to the target and those which are secondary accelerations forward to the 
target (D. Elliott, personal communication, April, 1992). The present data do not support this prediction.

one of the focal points of the present investigation was the role of visual feedback and the temporal constraints in the preparation of secondary submovements. As discussed at length previously in Experiment 2, this work has not been able to provide an adequate test of the dual submovement model's assumptions regarding this topic. However, I maintain my arguments against the model with respect to this issue (see Experiment 2 discussion).

This work has demonstrated that vision can be a potent influence on performance in goal-directed limb movements and on the kinematics underlying these actions (e.g., see also Elliott et al., 1991). This poses a problem for the dual submovement model in which the role of vision is minimized or given less than sufficient consideration.

Meyer and his colleagues (Meyer et al., 1990) have recently proposed an extension to the dual submovement model. The fundamental feature of the new model is that the number of submovements are no longer restricted to two. Rather, the Optimized Multiple Submovement Model assumes that a goaldirected limb movement can be composed of one, two, three, or more submovements. The model proposes the use of a parameter, the maximum submovement number, which has some "preset value" (Meyer et al., 1990, p. 213) depending on the 
situation at hand. I have a number of new questions and problems concerning this recent extension of the dual submovement model, specifically with respect to the implications associated with the maximum submovement parameter. For present purposes, no attempts are made to discuss these questions. However, one important point that should be mentioned is that the optimized multiple submovement model faces similar problems as its predecessor. Specifically, there have been no further attempts to integrate the role of vision into the model. Moreover, the basis of how corrective submovements are prepared remains unclarified. Given that the model now assumes that several submovements can occur, this issue becomes increasingly complex.

The nature of visual-motor regulation has received recent attention (e.g., Carson et al., 1992, in press; Elliott, 1992; Elliott et al., 1991; Pélisson et al., 1986). The purpose of employing the present kinematic analyses was to attempt to index different forms of trajectory modifications and infer the nature of the underlying mode of control. The use of "significant deviations", in addition to "zero crossings", provided a more sensitive measure of these adjustments (cf. Carson et al., 1992; Elliott et al., 1991; Meyer et al., 1988). However, as indicated in Experiment 2, even this measure may not be sufficiently discriminative. 
The reason for this may partly lie in the definition adopted here (cf. van Donkelaar \& Franks, 1991).

Significant deviations in the acceleration trace following peak velocity presumably reflect adjustments of the braking impulse without leading to a change in the sign of acceleration. If continuous visual control operates through graded adjustments of the muscle gain, or of the accelerative and braking impulses (Elliott, 1992; Elliott et al., 1991), then significant deviations may also reflect a mode of visuomotor control which is more continuous than discrete, as speculated previously. It does appear that this particular kinematic index is different from secondary accelerations, or zero crossings, which may be more reflective of discrete adjustments.

The absence of a visual influence on the number of adjustments to the trajectory in Experiment 2, despite the presence of a visually-based performance advantage, suggests that the present kinematic analysis was not sensitive to all forms of modificational processes which presumably contributed to better performance with vision. It appears that visual regulation of the limb movements may not necessarily be reflected in changes in the kinematic profiles, at least as presently defined. In this work, inference of a modificational process is primarily based upon the presence of defined fluctuations in the kinematic 
trajectories. It does not necessarily follow that in movement trajectories in which none of these deviations are present, that no amendments to the movement occurred. For example, in a movement in which no deviations or fluctuations are observed in the acceleration trace, it may be that an adjustment occurred in the form of a change in the peak magnitude of the the braking impulse. On an additional note, given the current definitions of continuous control le.g., see Elliott et al., 1991, and discussion above), perhaps an examination of changes in the rate of acceleration ("jerk") is warranted.

Models of limb control have frequently used the term "submovement" (e.g., Crossman \& Goodeve, 1963/1983; Keele, 1968, 1981; Meyer et al., 1988). Although the term seems relatively simple in meaning, its precise definition may require some further consideration. Precisely how should we define a submovement? Do the types of modifications enumerated here all qualify as submovements? My view is that they do not. I prefer to regard submovements as being equivalent to modifications such as secondary accelerations (i.e., zero crossings) and movement reversals. These adjustments are discrete and clearly identifiable in the acceleration, velocity, and displacement profiles. From this perspective, significant deviations (or more subtle changes in the rate of acceleration) would be considered as more 
continuous forms of regulatory adjustments, distinct from zero crossings and reversals.

On a final note, is there any necessity for models of limb control to constrain the number or type of movement corrections? Similarly, do we need to view visuomotor regulation as proceeding strictly in a discrete or continuous fashion? Based on the present data, I suggest that such constraints are neither necessary nor warranted. In the present experiments, there were movements which contained no evident corrections, discrete or continuous; some contained one or more discrete secondary accelerations or a reversal; some contained one or more significant deviations, and some contained a combination of these. There was evidence of both discrete and continuous control, based on present definitions. Thus, the mode of control, and the number and type of corrections probably vary from trial to trial, depending on the particular details of the situation at hand.

It certainly would not be a surprise for visuomotor regulation to exhibit this flexibility. After all, one inherent feature of motor control processes should be the ability to adapt to varying demands. The control of limb movements should be sensitive to a number of factors; these include, among others, the demands of the task, the goal of the performer, the task specific experience of the performer, 
and environmental conditions (e.g., see Marteniuk, Mackenzie, Jeannerod, Athenes, \& Dugas, 1987). 


\section{References}

Abrams, R.A., Meyer, D.E., \& Kornblum, S. (1990). Eye-hand coordination: Oculomotor control in rapid aimed limb movements. Journal of Experimental Psychology: Human Perception and Performance, 16, 248-267.

Annett, J., Golby, C.W. , \& Kay, H. (1958). The measurement of elements in an assembly task: The information output of the human motor system. The ouarterly Journal of Experimental Psychology, 10, 1-11.

Bard, C., Hay, L., \& Fleury, M. (1985). Role of peripheral vision in the directional control of rapid aiming movements. Canadian Journal of Psychology, 39, 151-161.

Beaubaton, D., \& Hay, L. (1986). Contribution of visual. information to feedforward and feedback processes in rapid pointing movements. Human Movement science, 5 . $19-34$.

Beggs, W.D.A., \& Howarth, C.I. (1988). Unpaced aiming - A control theory explanation. In A.C. Colley and J.R. Beech (Eds), cognition and action in skilled behaviour (pp.49-64). Amsterdam: Elsevier Science Publishers B.V.

Brooks, V.B. (1974). Some examples of programmed limb movements. Brain Research, 71, 299-308.

Carlton, L.G. (1979). Control processes in the production of discrete aiming responses. Journal of Human Movement studies, 5, 115-124.

Carlton, L.G. (1981). Processing visual feedback information for movement control. Journal of Experimental Psychology: Human Perception and Performance, 7, $1019-$ 1030 .

Carlton, L.G. (1992). Visual processing time and the control of movement. In L. Proteau and D. Elliott (Eds), Vision and motor control ( $\mathrm{pp} .3-31$ ). Amsterdam: Elsevier Science Publishers B.V.

Carson, R.G., Chua, R., Elliott, D., \& Goodman, D. (1990). The contribution of vision to asymmetries in manual aiming. Neuropsychologia, 28, 1215-1220. 
Carson, R.G., Goodman, D., Chua, R., \& Elliott, D. (in press). Asymmetries in the regulation of visually guided aiming. Journal of Motor Behavior.

Carson, R.G., Goodman, D., \& Elliott, D. (1992). Asymmetries in the discrete and pseudocontinuous regulation of visually guided reaching. Brain and Cognition, 18, 169191.

Carson, R.G., Goodman, D., Elliott, D., \& Chua, R. (1991, June). Evidence for non-functional, visually based modification of movement trajectories: Implications for manual asymmetries. Paper presented at the North American Society for the Psychology of Sport and Physical Activity Conference, Asilomar, California.

Conti, P., et Beaubaton, D. (1976). Utilisation des informations visuelles dans le controle du mouvement: etude de la precision des pointages chez l'homme. Le Travail Humain, 39, 19-32.

Crossman, E.R.F., \& Goodeve, P.J. (1963). Feedback control of hand-movement and Fitts' law. Paper presented at the meeting of the Experimental Psychology Society, Oxford, July 1963. Published in The Quarterly Journal of Experimental Psychology, 1983, 35A, 251-278.

Elliott, D. (1988). The influence of visual target and limb information on manual aiming. Canadian Journal of Psychology, 42, 57-68.

Elliott, D. (1990). Intermittent visual pickup and goal directed movement: A review. Human Movement Science, 2, 531-548.

Elliott, D. (1992). Intermittent versus continuous control of manual aiming movements. In $L$. Proteau and $D$. Elliott (Eds), Vision and motor control (pp. 33-48). Amsterdam: Elsevier Science Publishers B.V.

Elliott, D., \& Allard, F. (1985). The utilization of visual feedback information during rapid pointing movements. The Ouarterly Journal of Experimental Psychology, 37A, $407-425$.

Elliott, D., Carson, R.G., Goodman, D., \& Chua, R. (1991). Discrete vs. Continuous visual control of manual aiming. Human Movement Science, 10, 393-418. 
Elliott, D., \& Madalena, J 1987 The influence of premovement visual information on manual aiming The ouarterly Journal of Experimental Psychology, 39A, 541559 .

Fitts, P.M (1954). The information capacity of the human motor system in controlling the amplitude of movement Journal of Experimental Psychology, 47, 381-391.

Fitts, P.M , \& Peterson, J.R (1964) Information capacity of discrete motor responses Journal of Experimental Psychology, 67, 103-112

Hancock, P.A, \& Newell, K M 1985 The movement speedaccuracy relationship in space-time In $\mathrm{H}$ Heuer, $\mathrm{U}$ Kleinbeck, and $\mathrm{K}-\mathrm{H}$. Schmidt (Eds), Motor behaviour. Programming, control and acquisition (pp 153-188 Berlin: Springer-Verlag

Heuer, H. (1984) On re-scalability of force and time in aiming movements Psychological Research, 46, 73-86

Howarth, C.I., \& Beggs, W D A (1985) The control of movements by multisensory information In $\mathrm{H}$ Heuer, $U$ Kleinbeck, and $\mathrm{K}-\mathrm{H}$ Schmidt (Eds), Motor behaviour: Programming, control and acquisition pp 125-151

Berlin: Springer-Verlag

Howarth, C.I., Beggs, W.D A, \& Bowden, J M (1971) The relationship between speed and accuracy of movement aimed at a target Acta Psychologica, 35, 207-218

Jagacinski, R J , Repperger, D W , Moran, M S , Ward, S.L , \& Glass, B (1980) Fitts' law and the microstructure of rapid discrete movements. Journal of Experimental Psychology: Human Perception and Performance, 6, 309320

Jeannerod, M (1984) The timing of natural prehension movements Journal of Motor Behavior, 16, 235-254

Jeannerod, M. (1988) The neural and behavioural organization of goal-directed movements oxford: Clarendon Press

Jeannerod, M., \& Prablanc, C (1983) Visual control of reaching movements in man In $\mathrm{J} E$ Desmedt Ed), Motor control mechanisms in health and disease pp 13-29) New York Raven Press 
Keele, S W (1968). Movement control in skilled performance Psychological Bulletin, 70, 387-404

Keele, S W. (1981) Behavioural analysis of movement In V.B. Brooks (Ed.), Handbook of physiology, Section I: The nervous system, Vol. II: Motor control part 2 (pp 1391-1414). Baltimore: Williams \& Wilkins

Langolf, G D., Chaffin, D.B , \& Foulke, J A (1976) An investigation of Fitts' law using a wide range of movement amplitudes Journal of Motor Behavior, 8 , 113128

Mackenzie, C L., Marteniuk, R. G., Dugas, C, Liske, D , \& Eickmeier, B. (1987) Three-dimensional movement trajectories in Fitts' task: Implications for control The ouarterly Journal of Experimental Psychology, 39A, $629-647$

Marteniuk, R.G., Mackenzie, C L., Jeannerod, M, Athenes, S , \& Dugas, C (1987) Constraints on human arm movement trajectories Canadian Journal of Psychology, 41, 365378

Meyer, D E, Abrams, R A, Kornblum, S, Wright, C E , \& Smith, J.E K (1988) Optimality in human motor performance Ideal control of rapid aimed movements Psychological Review, 95, 340-370

Meyer, D.E., Smith, J.E.K, Kornblum, S , Abrams, R A, \& Wright, C E (1990) Speed-accuracy tradeoffs in aimed movements: Toward a theory of rapid voluntary action In $M$ Jeannerod (Ed), Attention and performance XIII (pp 173-226) Hillsdale Lawrence Erlbaum

Meyer, D E, Smith, J E K., \& Wright, C E 1982 Models for the speed and accuracy of aimed movements Psychological Review, 89, 449-482

Milner, T.E., \& Ijaz, M M $(1990$ The effect of accuracy constraints on three-dimensional movement kinematics Neuroscience, $35,365-374$

Newell, K M (1980 The speed-accuracy paradox in movement control: Errors of time and space In G E Stelmach and $\mathrm{J}$. Requin (Eds.), Tutorials in motor behavior pp 501510). Amsterdam: Elsevier Science Publishers B V 
Oldfield, R.C (1971) The assessment and analysis of handedness: The Edinburgh inventory Neuropsychologia, 2. 97-113.

Paillard, J. (1980) The multi channeling of visual cues and the organization of a visually guided response In G E. Stelmach and $\mathrm{J}$ Requin (Eds), Tutorials in motor behavior (pp 259-279). Amsterdam: Elsevier Science Publishers B.V

Paillard, J. (1982) The contribution of peripheral and central vision to visually guided reaching In D Ingle, M.A. Goodale, and R Mansfield (Eds), Advances in the analysis of visual behaviour pp 367-385 Cambridge: MIT Press

Pélisson, D., Prablanc, C , Goodale, M A , \& Jeannerod, M (1986). Visual control of reaching movements without vision of the limb. II Evidence of fast unconscious processes correcting the trajectory of the hand to the final position of a double-step stimulus Experimental Brain Research, 62, 303-311

Pepper, R L., \& Herman, L M 1970 . Decay and interference effects in the short-term retention of a discrete motor act Journal of Experimental Psychology Monograph, 83, $1-18$.

Prablanc, C , Echallier, J E, Jeannerod, M , \& Komilis, E (1979). Optimal response of eye and hand motor systems in pointing at a visual target II Static and dynamic visual cues in the control of hand movement Biological Cybernetics, 35, 183-187

Prablanc, C., Echallier, J.E, Komilis, E, \& Jeannerod, M (1979) Optimal response of eye and hand motor systems in pointing at a visual target I spatio-temporal characteristics of eye and hand movements and their relationships when varying the amount of visual

information. Biological Cybernetics, 35, 113-124

Prablanc, C , Pélisson, D., \& Goodale, M A $(1986$ Visual control of reaching movements without vision of the limb I. Role of retinal feedback of target position in guiding the hand Experimental Brain Research, 62, 293302

Schmidt, R A, Sherwood, D E, Zelaznik, H N , \& Leikind, B.J. 1985) Speed-accuracy trade-offs in motor 
behavior: Theories of impulse variability In $\mathrm{H}$ Heuer, $\mathrm{U}$ Kleinbeck, and $\mathrm{K}-\mathrm{H}$ Schmidt (Eds), Motor behaviour. programming, control and acquisition (pp 79-123 Berlin: Springer-Verlag

Schmidt, R.A., Zelaznik, H.N , \& Frank, J S (1978). Sources of inaccuracy in rapid movement. In $G E$ Stelmach (Ed.), Information processing in motor control and learning (pp. 183-203) New York: Academic Press

Schmidt, R.A., Zelaznik, $\mathrm{H} N$, Hawkins, B, Frank, J S , \& Quinn, J.T (1979) Motor output variability: A theory for the accuracy of rapid motor acts Psychological Review, 86, 415-451.

Sherwood, D.E (1986). Impulse characteristics in rapid movement: Implications for impulse-variability models Journal of Motor Behavior, 18, 188-214.

Soechting, J.F. (1984). Effect of target size on spatial and temporal characteristics of a pointing movement in man Experimental Brain Research, 54, 121-132

Todor, J.I, Cisneros, J. 1985) Accomodation to increased accuracy demands by the right and left hands. Journal of Motor Behavior, 17, 355-372

Todor, J.I., \& Smiley, A L (1985). Performance differences between the hands: Implications for studying disruption to limb praxis In E A Roy (Ed), Neuropsychological studies of apraxia and related disorders (pp. 309-344 Amsterdam: Elsevier Science Publishers B V

van Donkelaar, P , \& Franks, I M $(1991$ The effects of changing movement velocity and complexity on response preparation: Evidence from latency, kinematic, and EMG measures. Experimental Brain Research, 83, 618-632

von Holst, E. (1954) Relations between the central nervous system and the peripheral organs Animal Behaviour, 2 , $89-94$

Wallace, S A., \& Newell, K M (1983 Visual control of discrete aiming movements The Quarterly Journal of Experimental Psychology, 35A, 311-321

Woodworth, R S (1899 The accuracy of voluntary movement Psychological Review, 3 (monograph supplement 3, whole no.13), 1-114 
Worringham, C.J (1991) Variability effects on the internal structure of rapid aiming movements. Journal of Motor Behavior, 23, 75-85.

Wright, C.E. \& Meyer, DE $(1983$ Conditions for a linear speed-accuracy trade-off in aimed movements The Quarterly Journal of Experimental Psychology, 35A, 279296.

Young, R.P., \& Zelaznik, H N. (1992) The visual control of aimed hand movements to stationary and moving targets Acta Psychologica, 79, 59-78.

Zelaznik, H N , Hawkins, B , \& Kisselburgh, L (1983) Rapid visual feedback processing in single-aiming movements. Journal of Motor Behavior, 15, 217-236

Zelaznik, H.N., Schmidt, R.A, \& Gielen, S C A M (1986) Kinematic properties of rapid aimed hand movements. Journal of Motor Behavior, 18, 353-372 
Appendix A

Nomenclature 


\section{Summary of Terms}

VC - Vision-Cursor condition

NC - No Vision-Cursor condition

FHV - First-Half Vision condition

LHV - Last-Half Vision condition

VXFH - First-Half Vision trials within a block of VC trials VXLH - Last-Half Vision trials within a block of VC trials NXFH - First-Half Vision trials within a block of NC trials NXLH - Last-Half Vision trials within a block of NC trials MT - Movement Time

AE - Absolute Error

MODS - Total number of modifications

TPV/MT - Time to peak velocity as a proportion of MT

TPV-END - Time from peak velocity to the end of the movement PVDISP/DIST - Proportion of target distance travelled at time of peak velocity

SDPV - Significant deviation in the acceleration profile prior to time of peak velocity PVSD - Significant deviation in the acceleration profile following time of peak velocity $\mathrm{zC}$ - Negative to positive zero crossing in the acceleration profile REVS - Reversal in the direction of movement 
Appendix B

Kinematic Profiles

131 


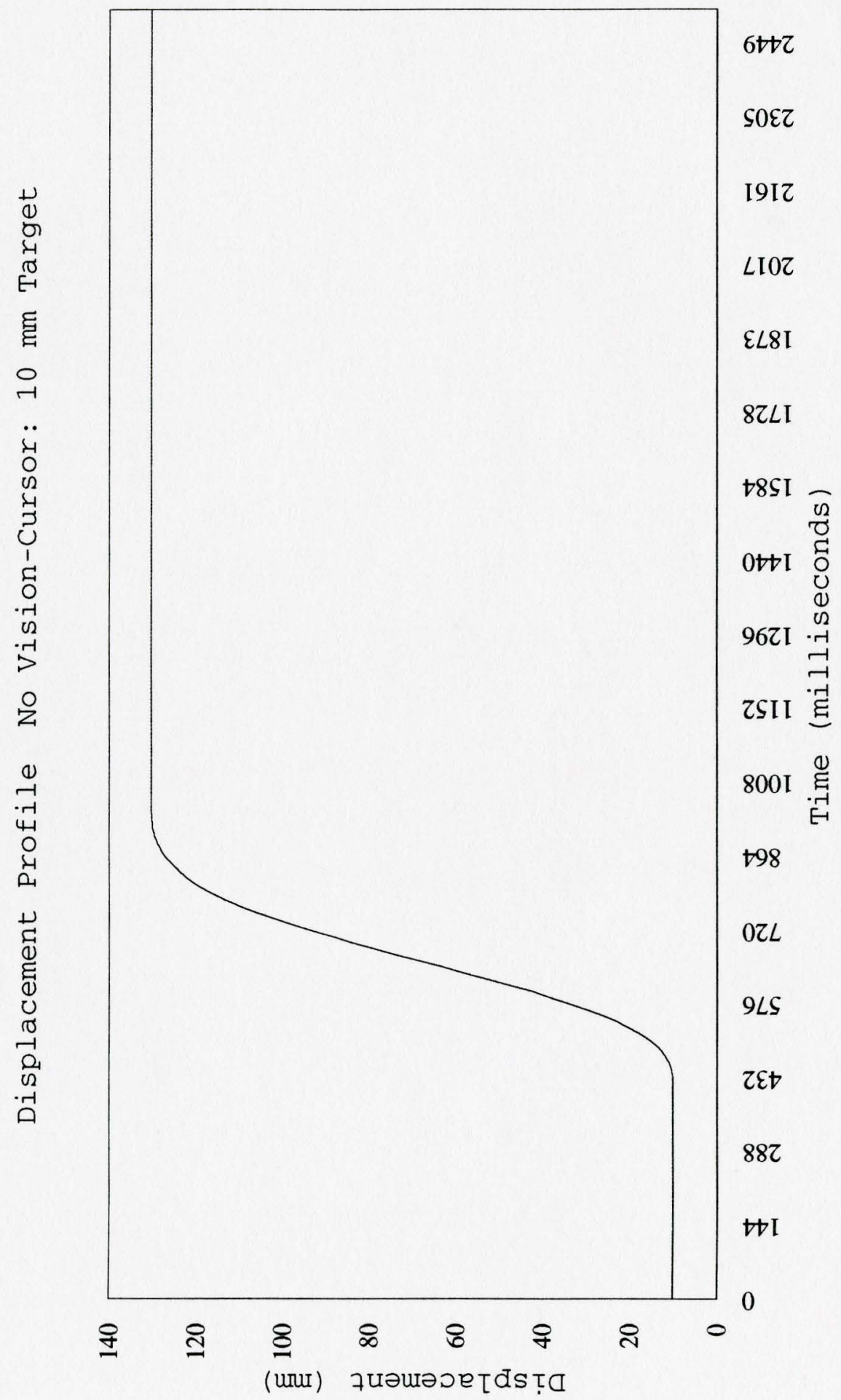




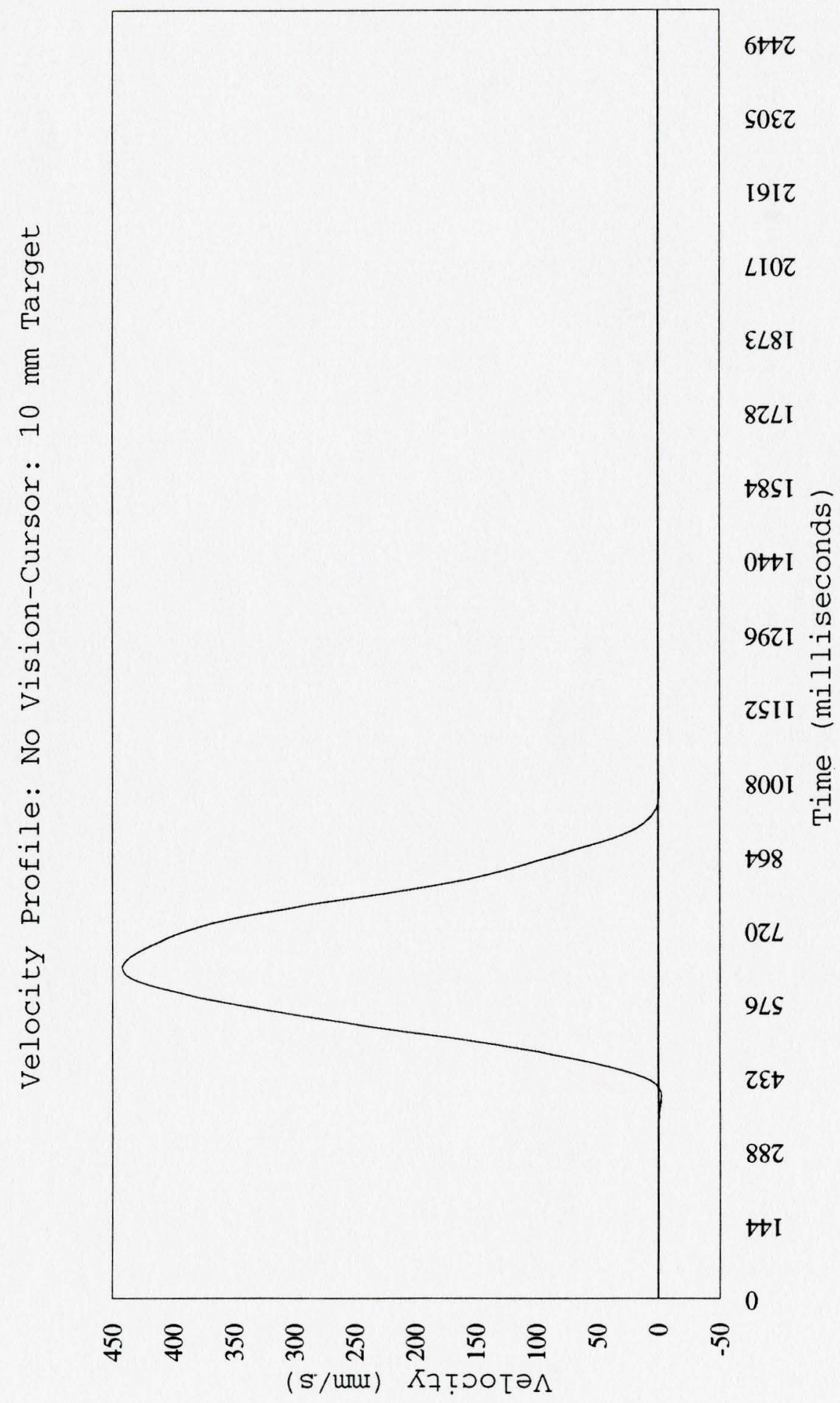




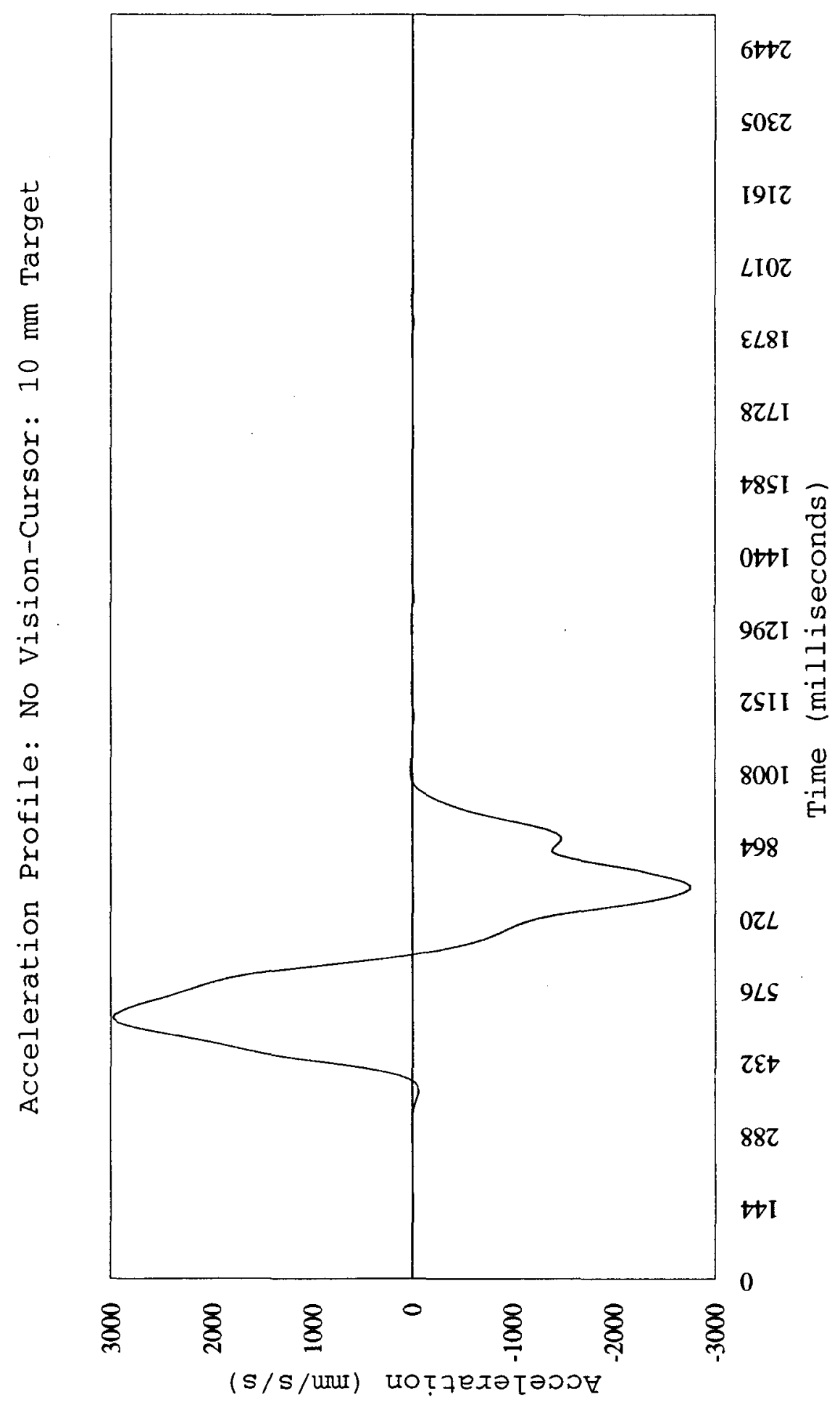




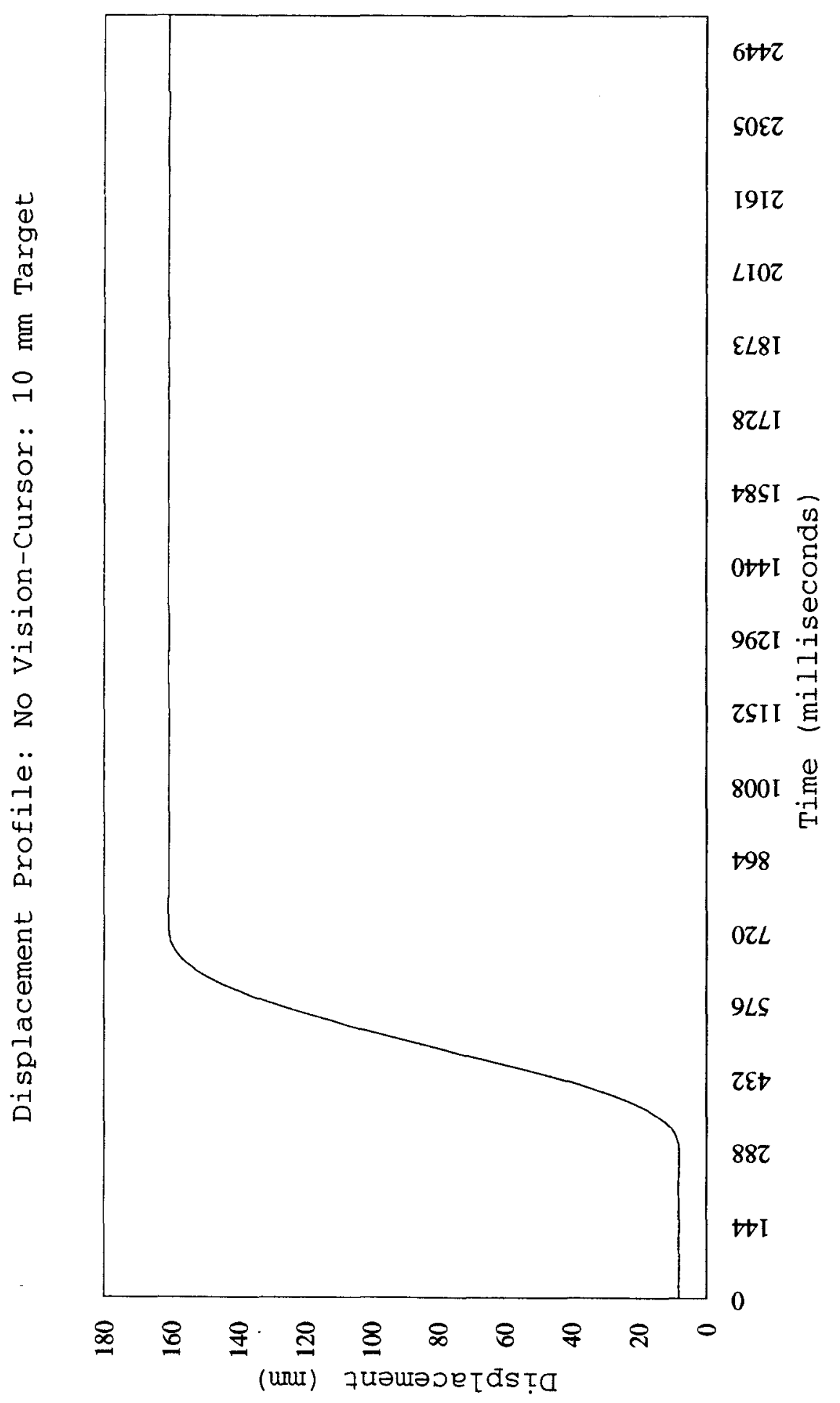




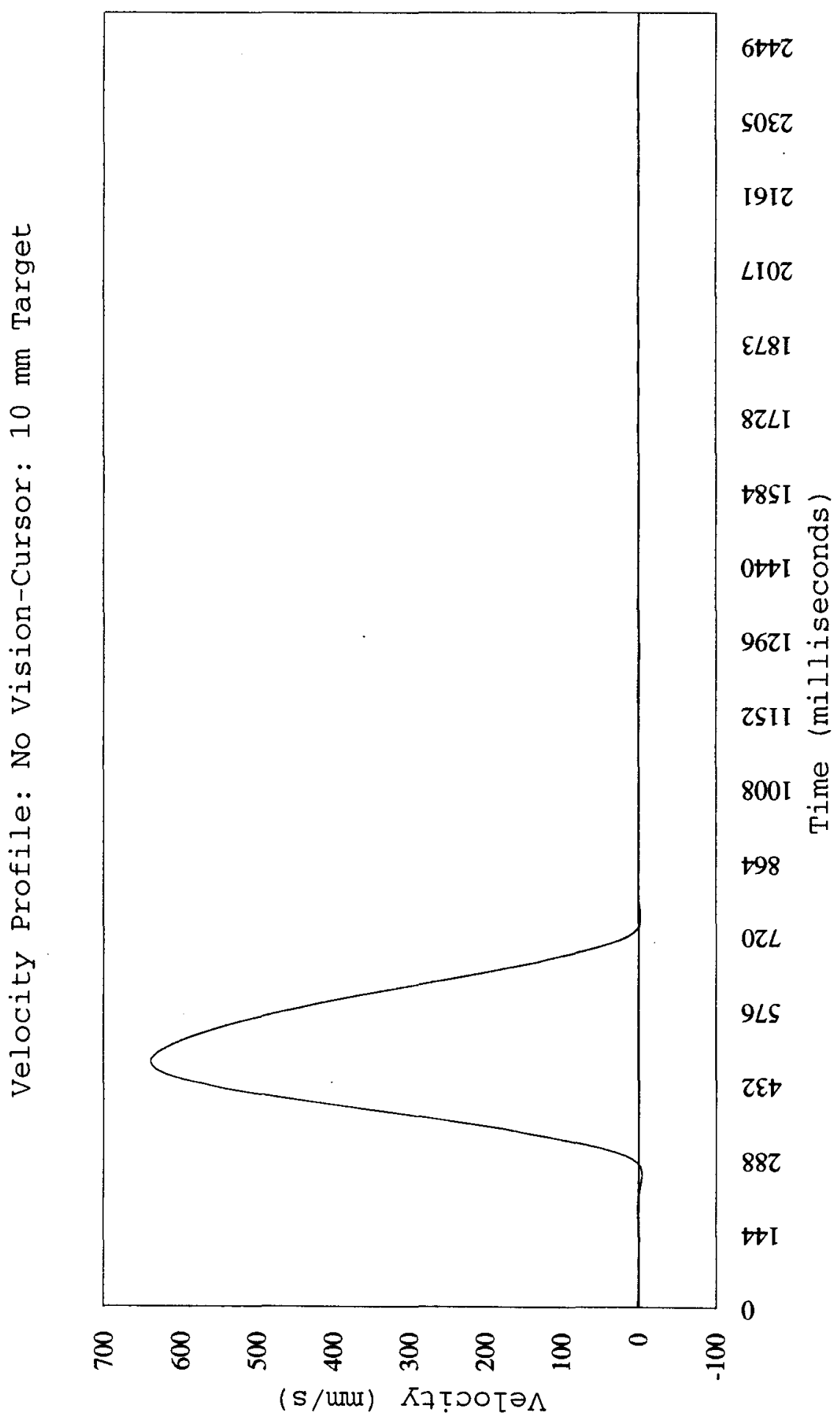




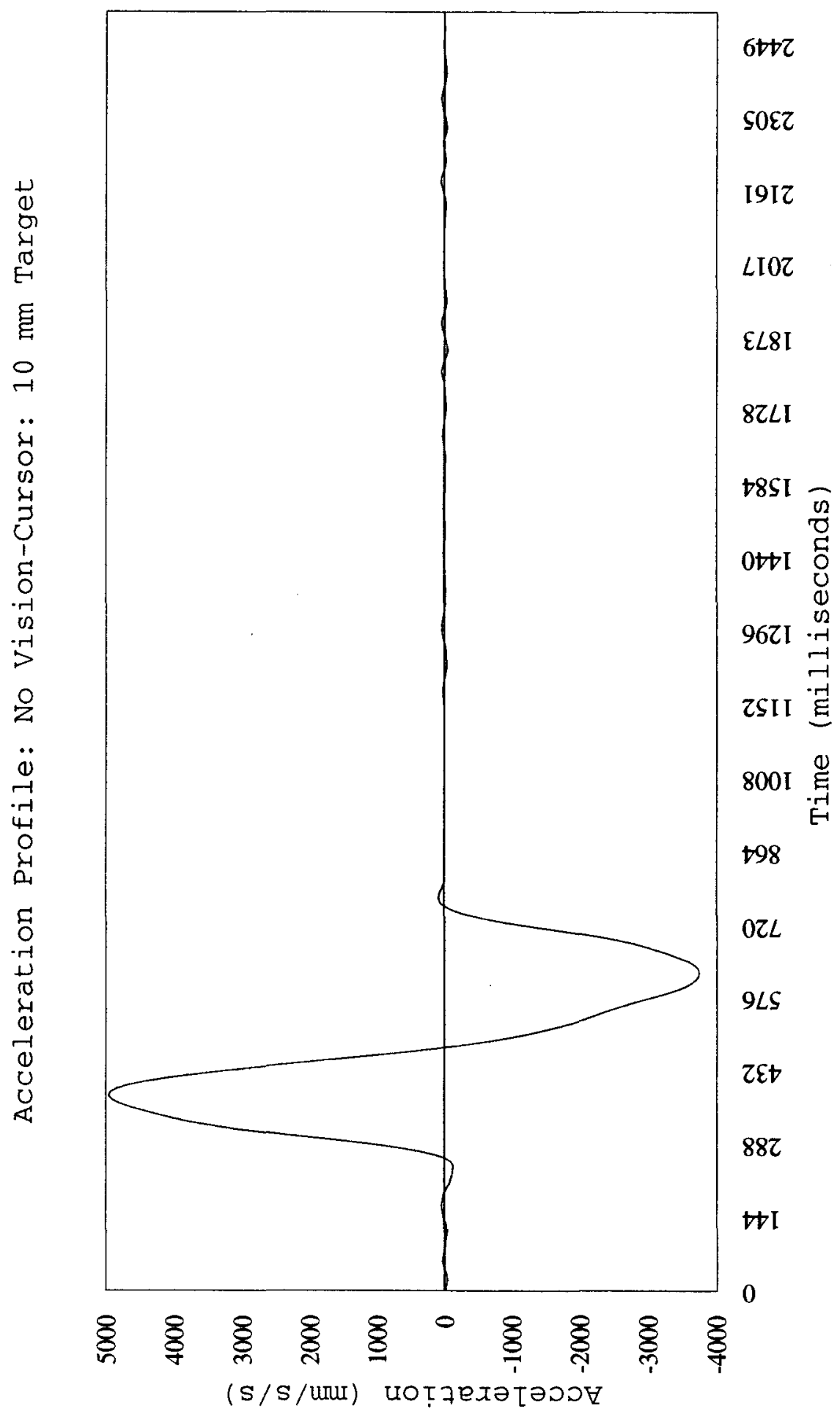




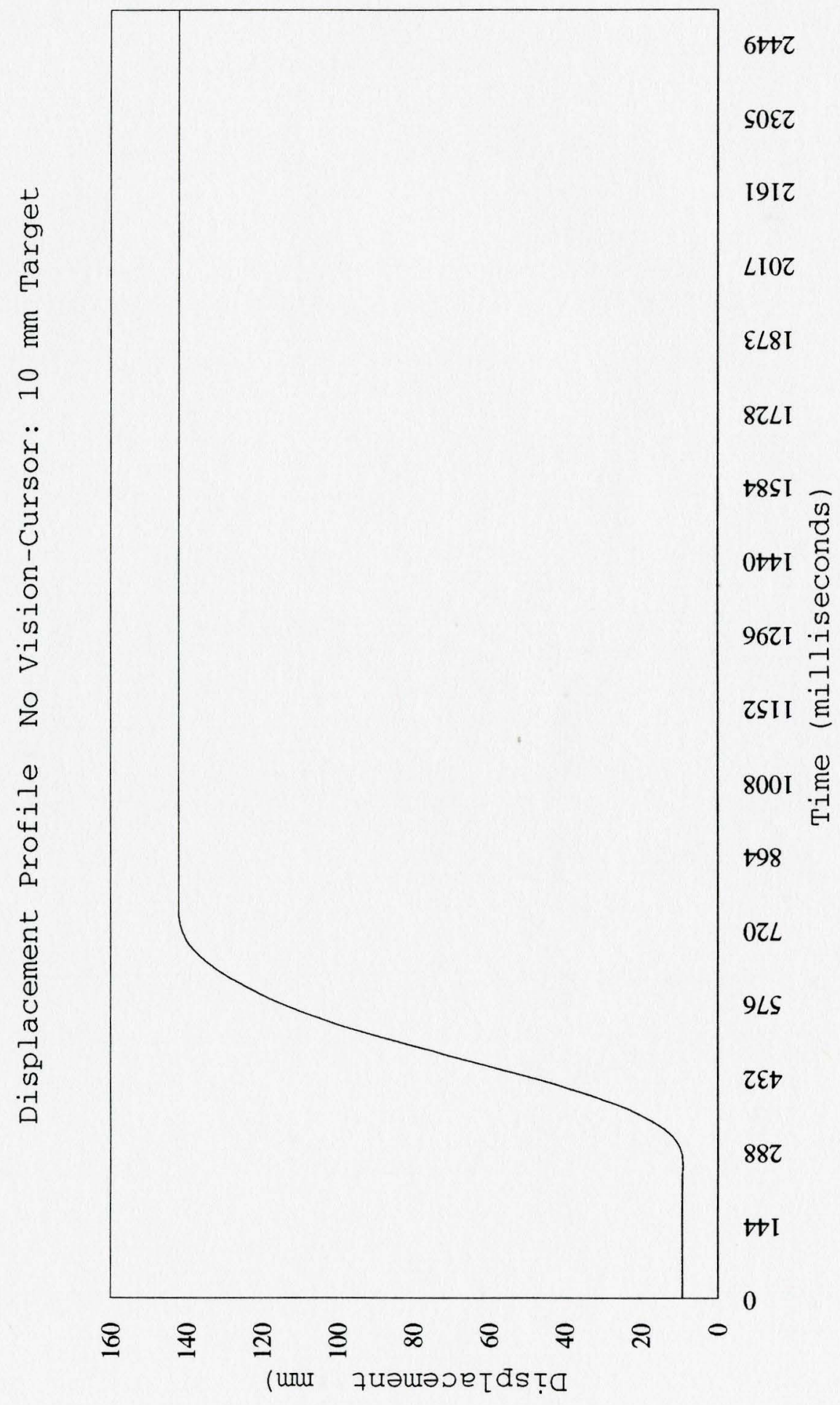




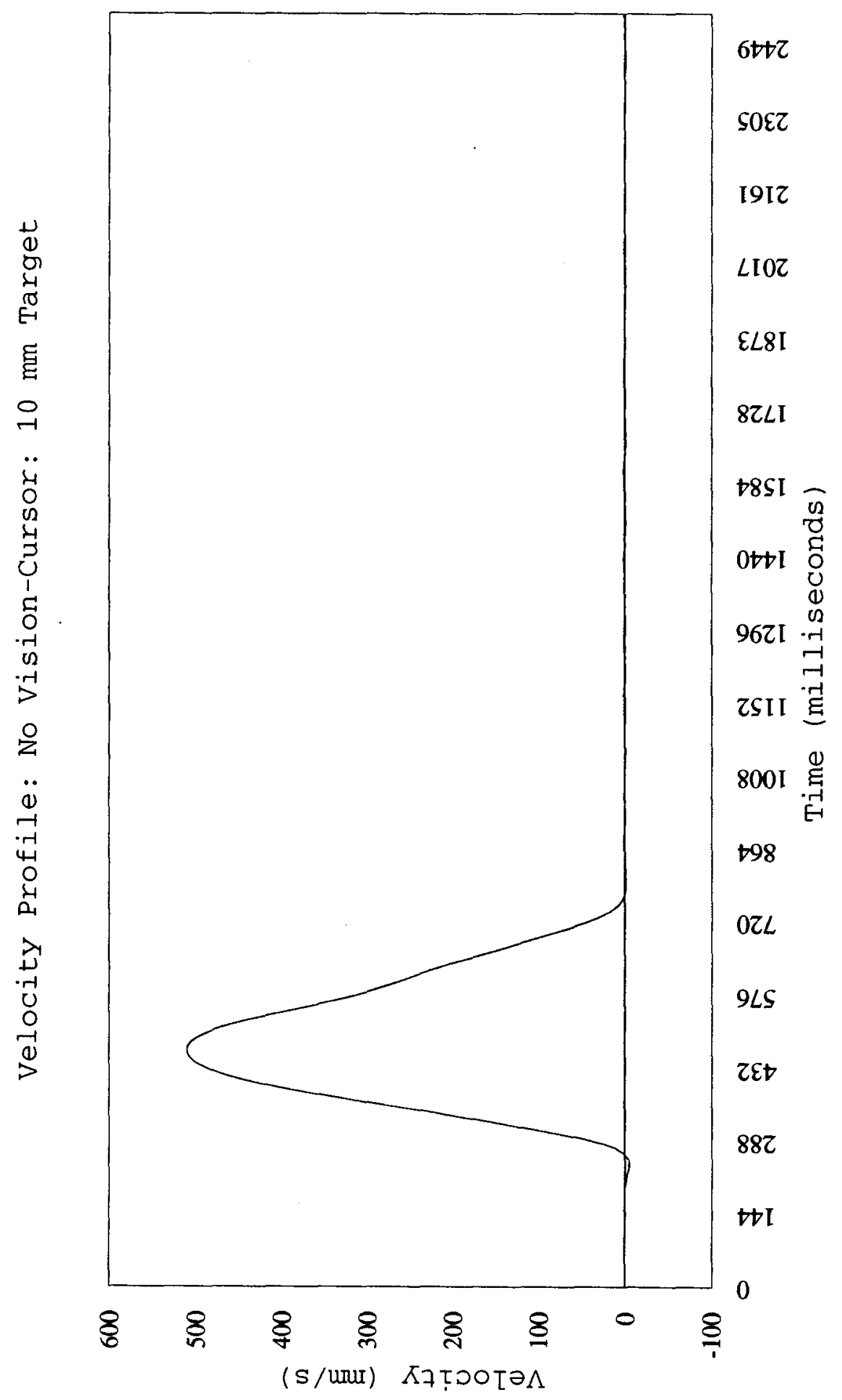




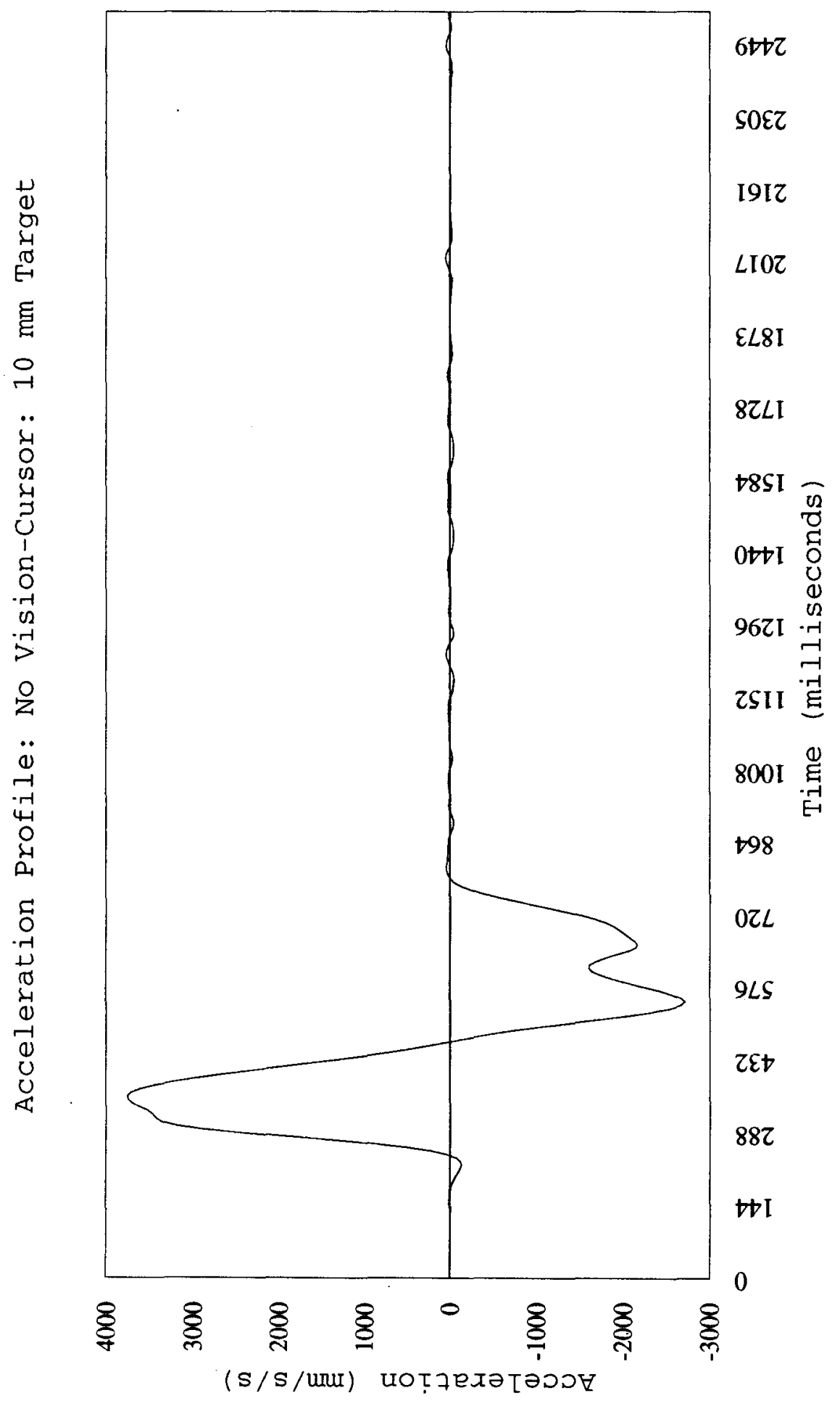




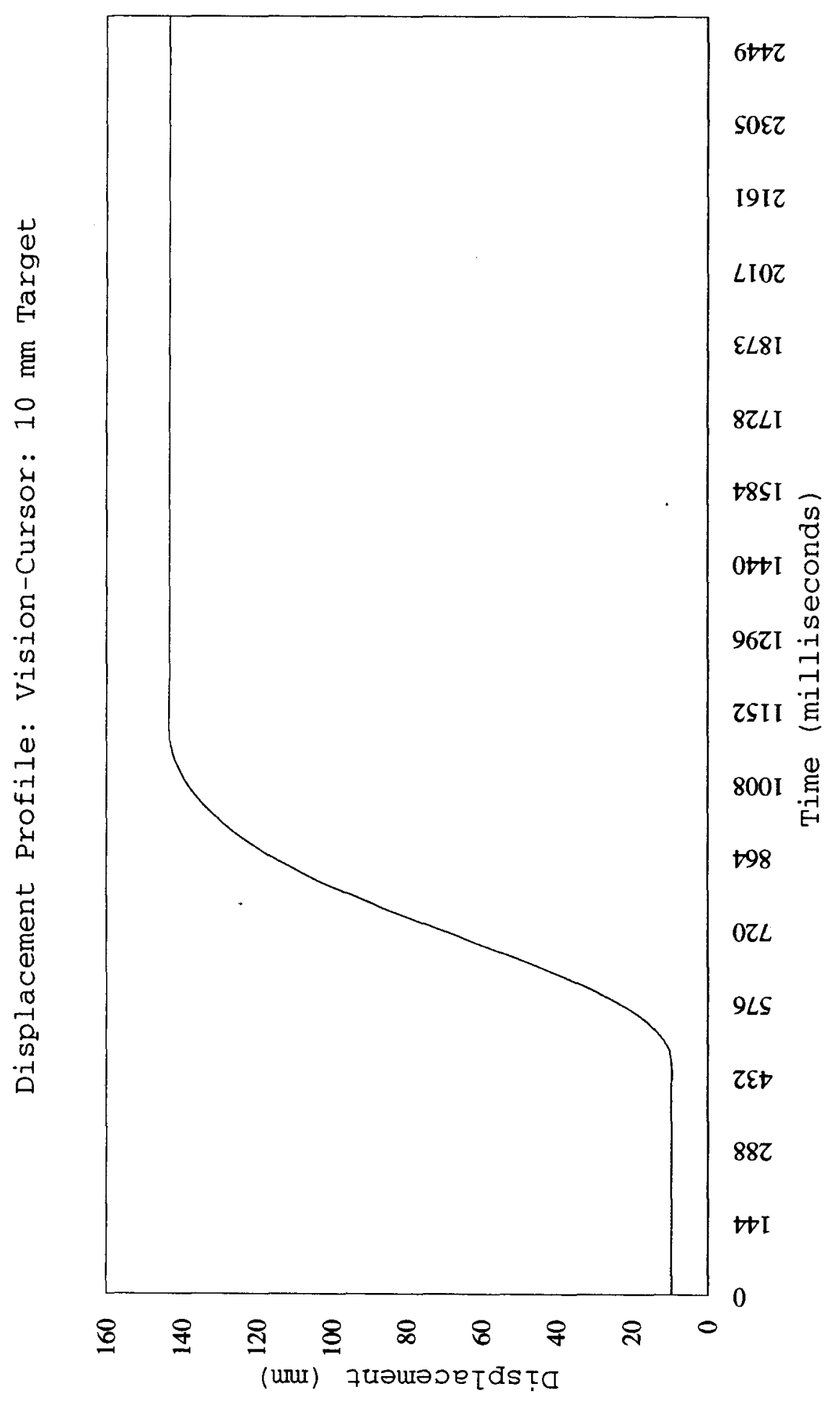




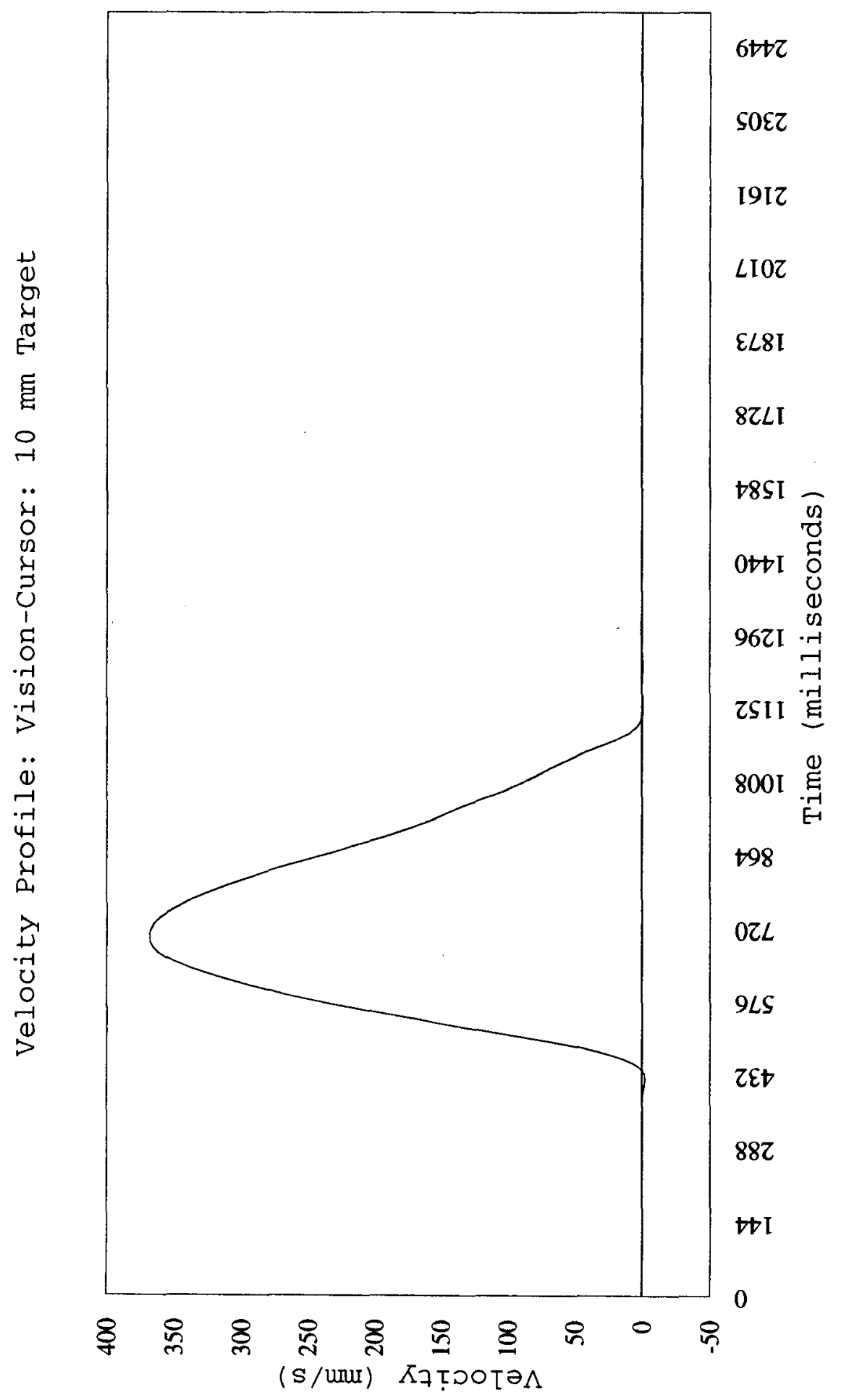




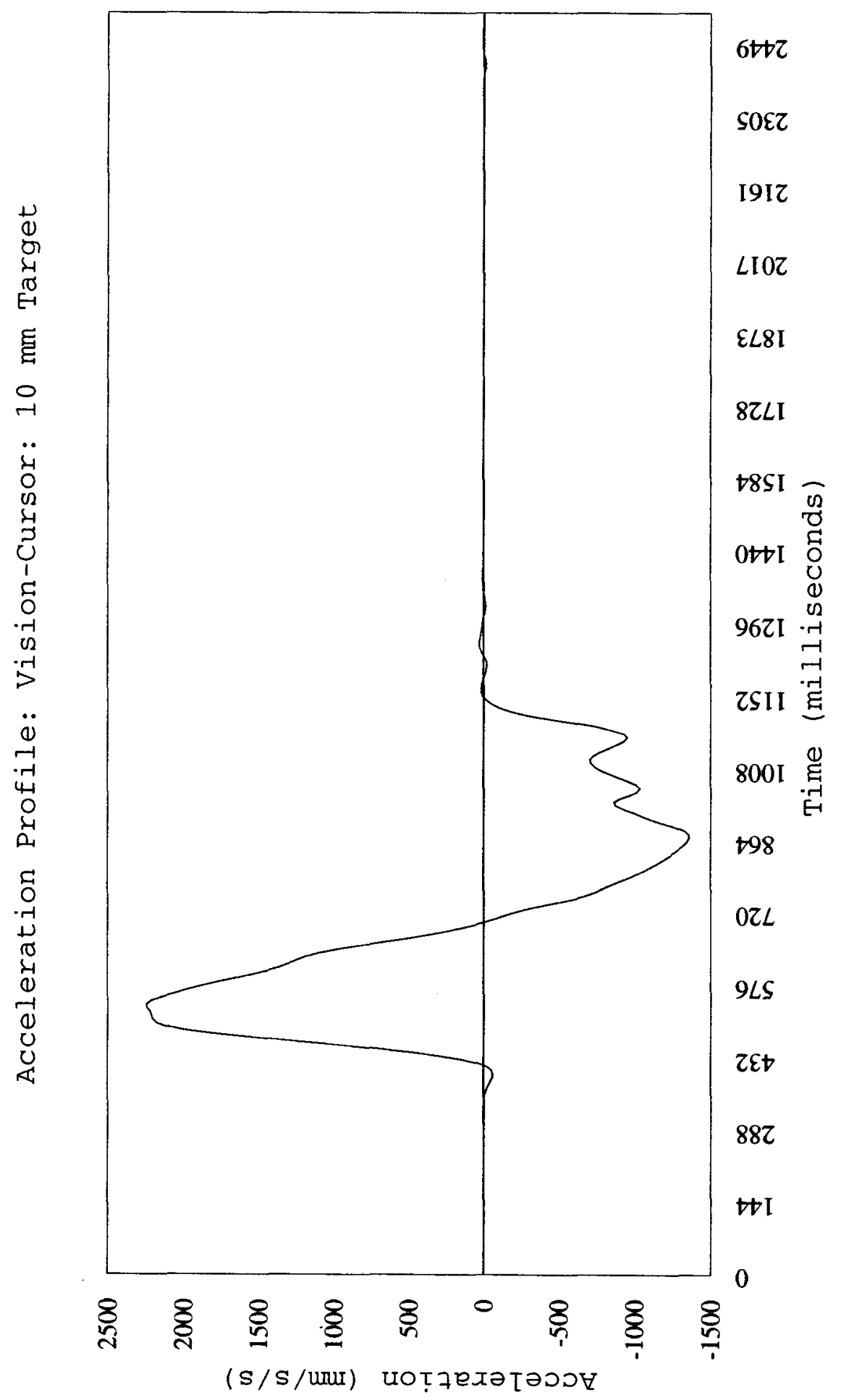




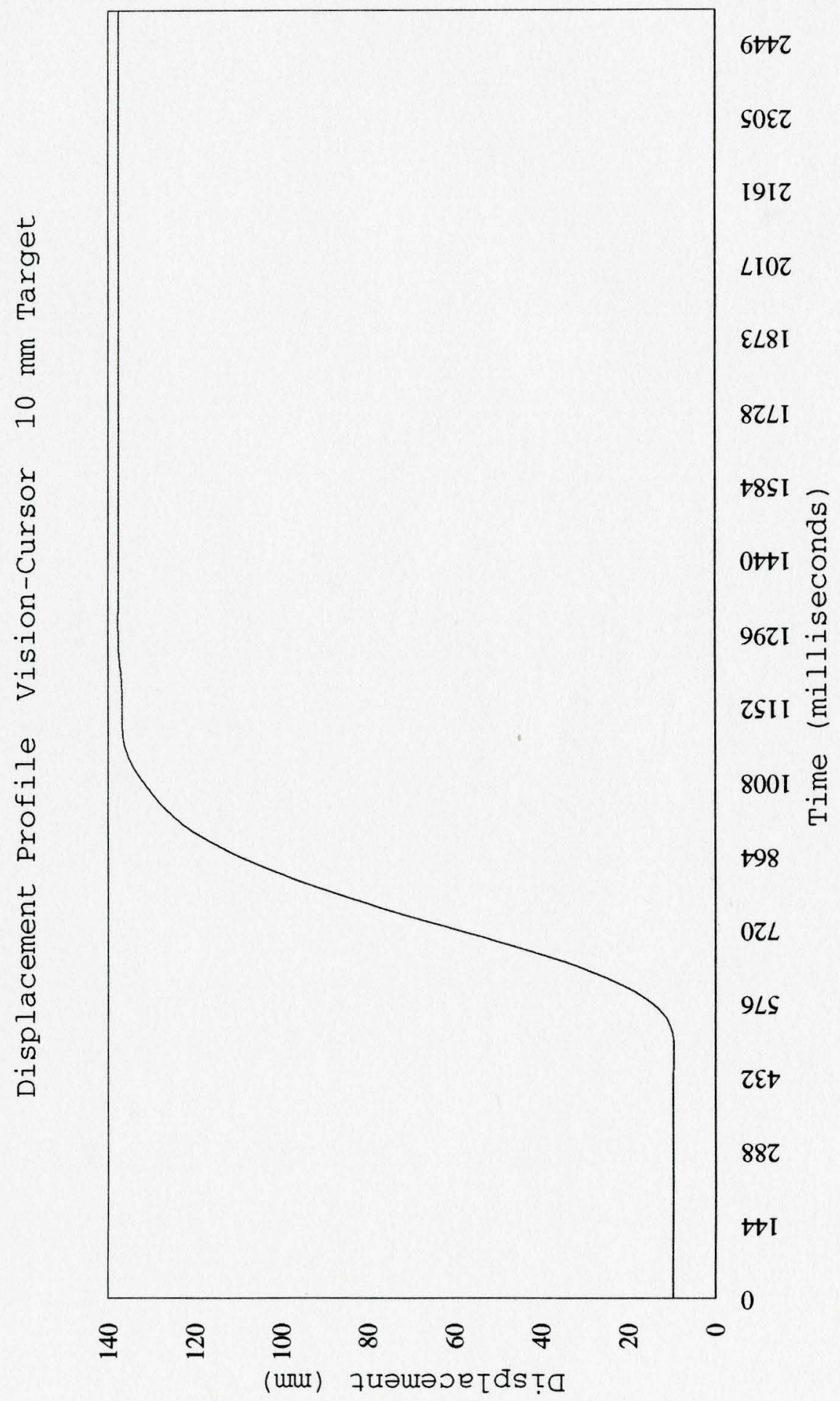


145

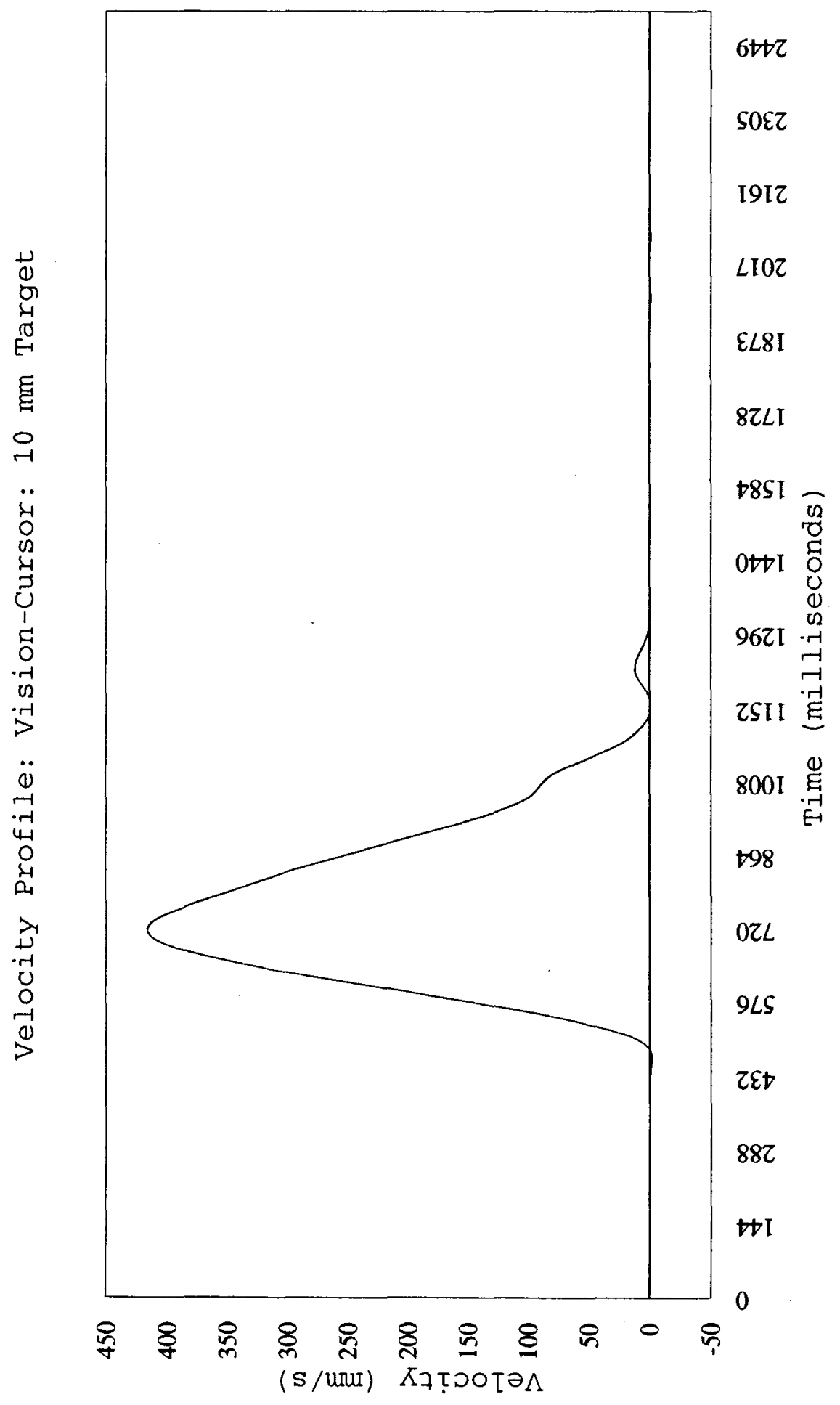




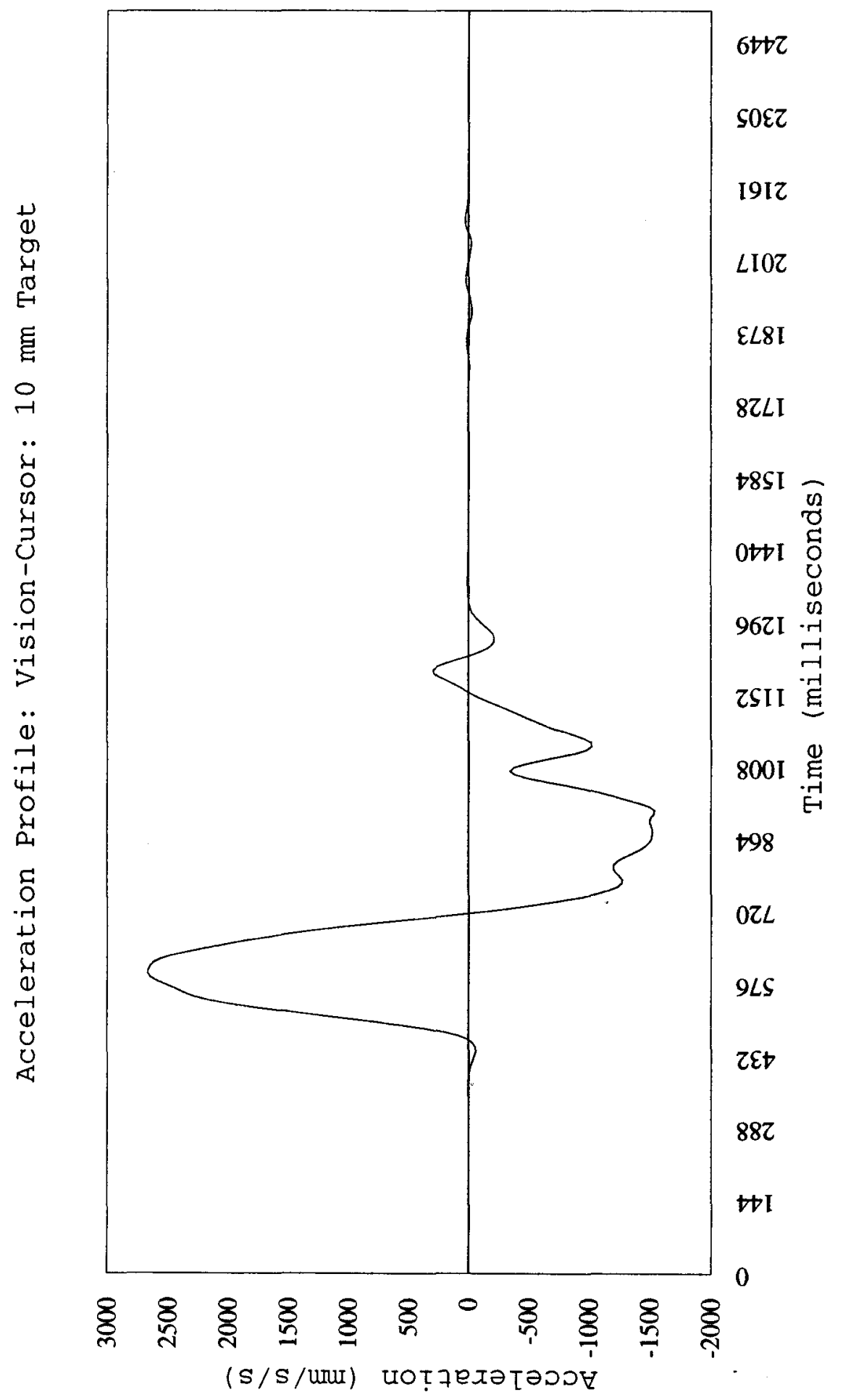




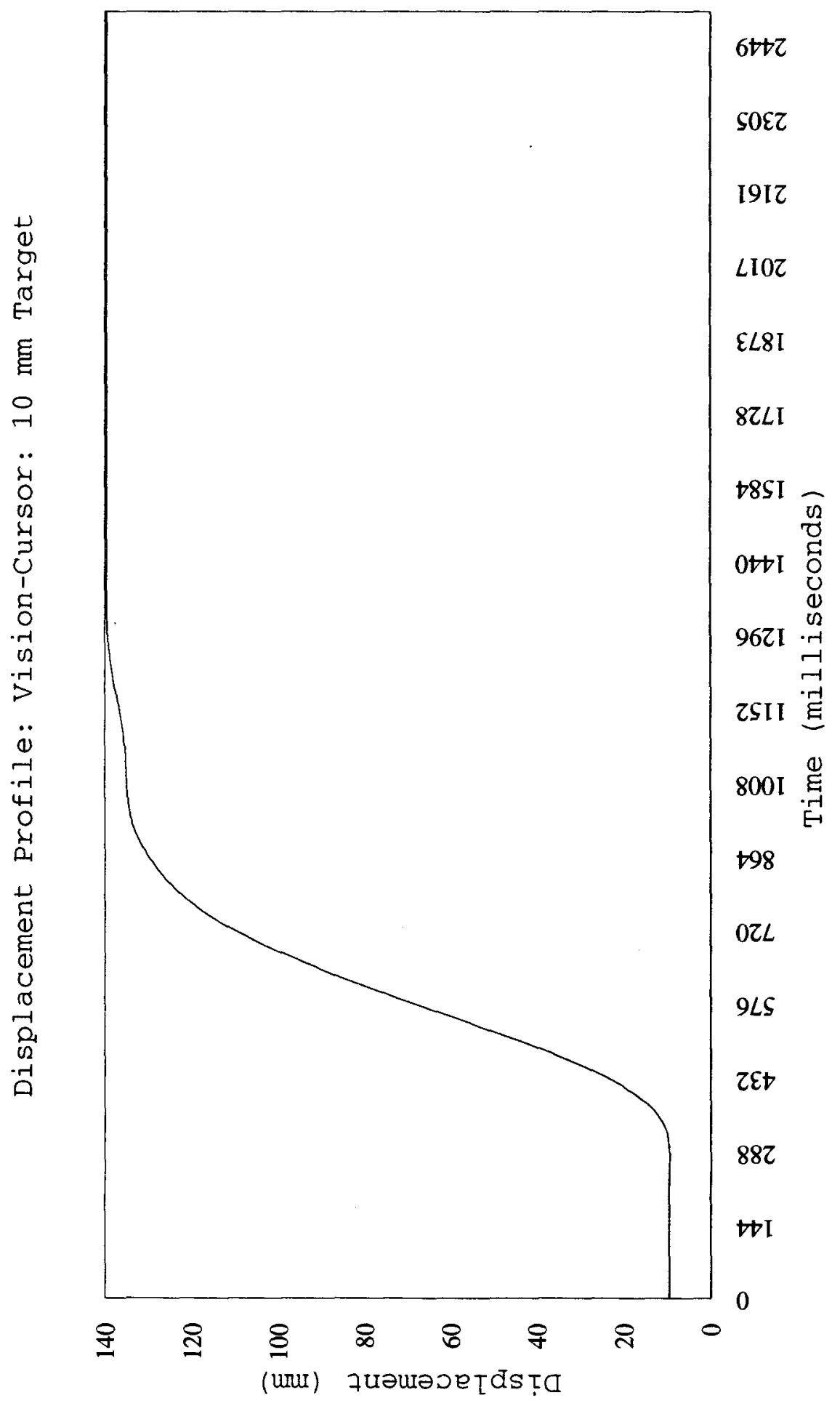




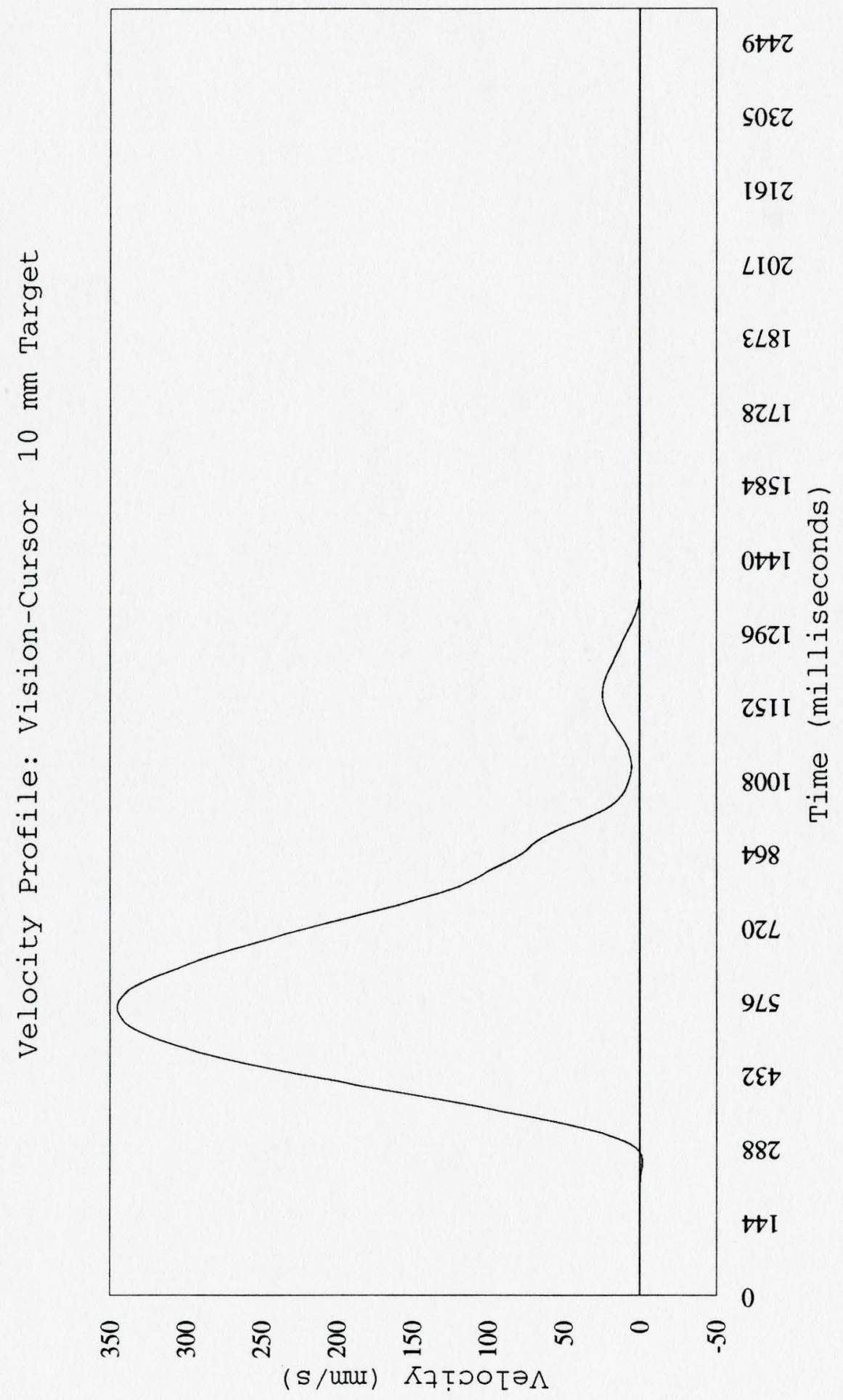




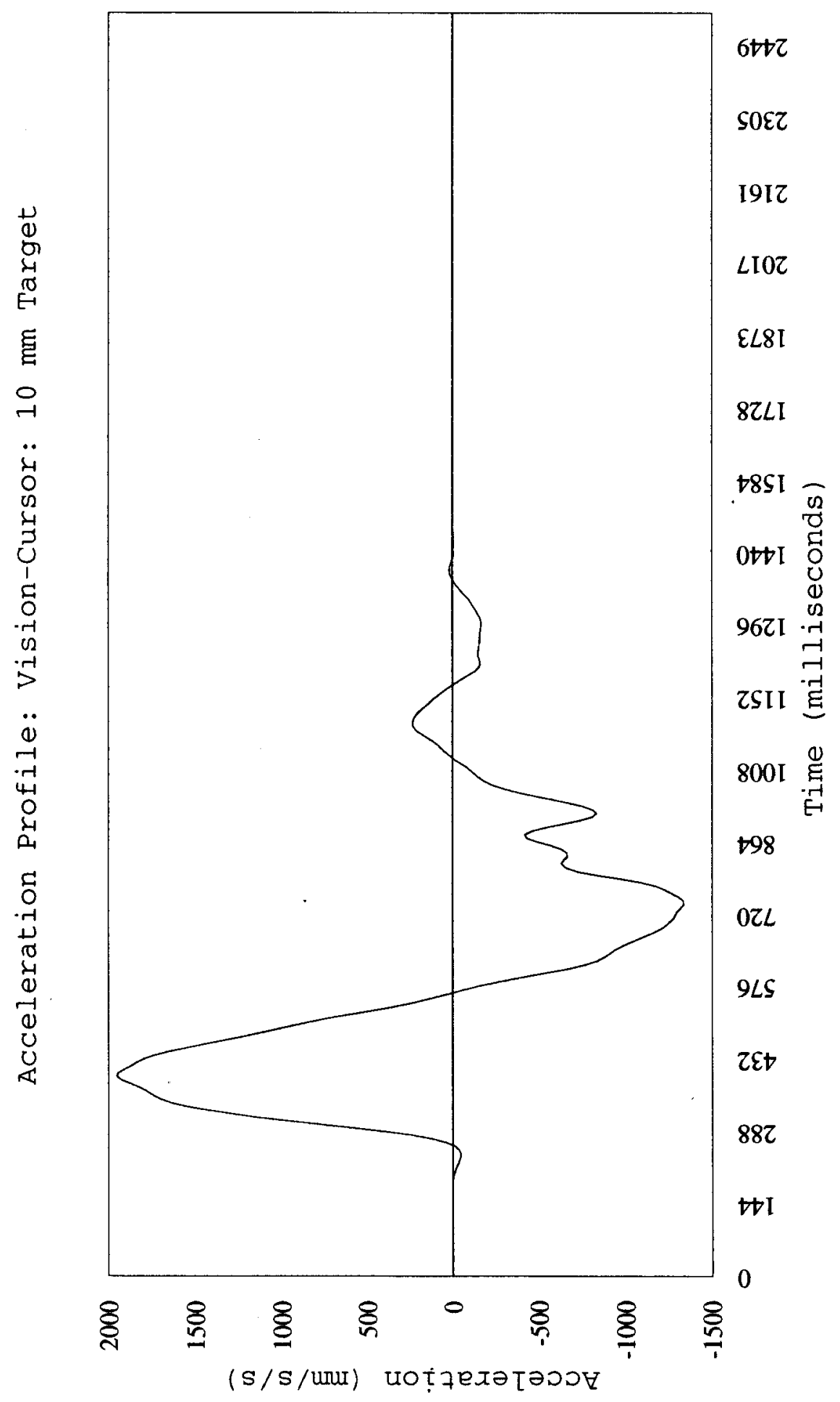




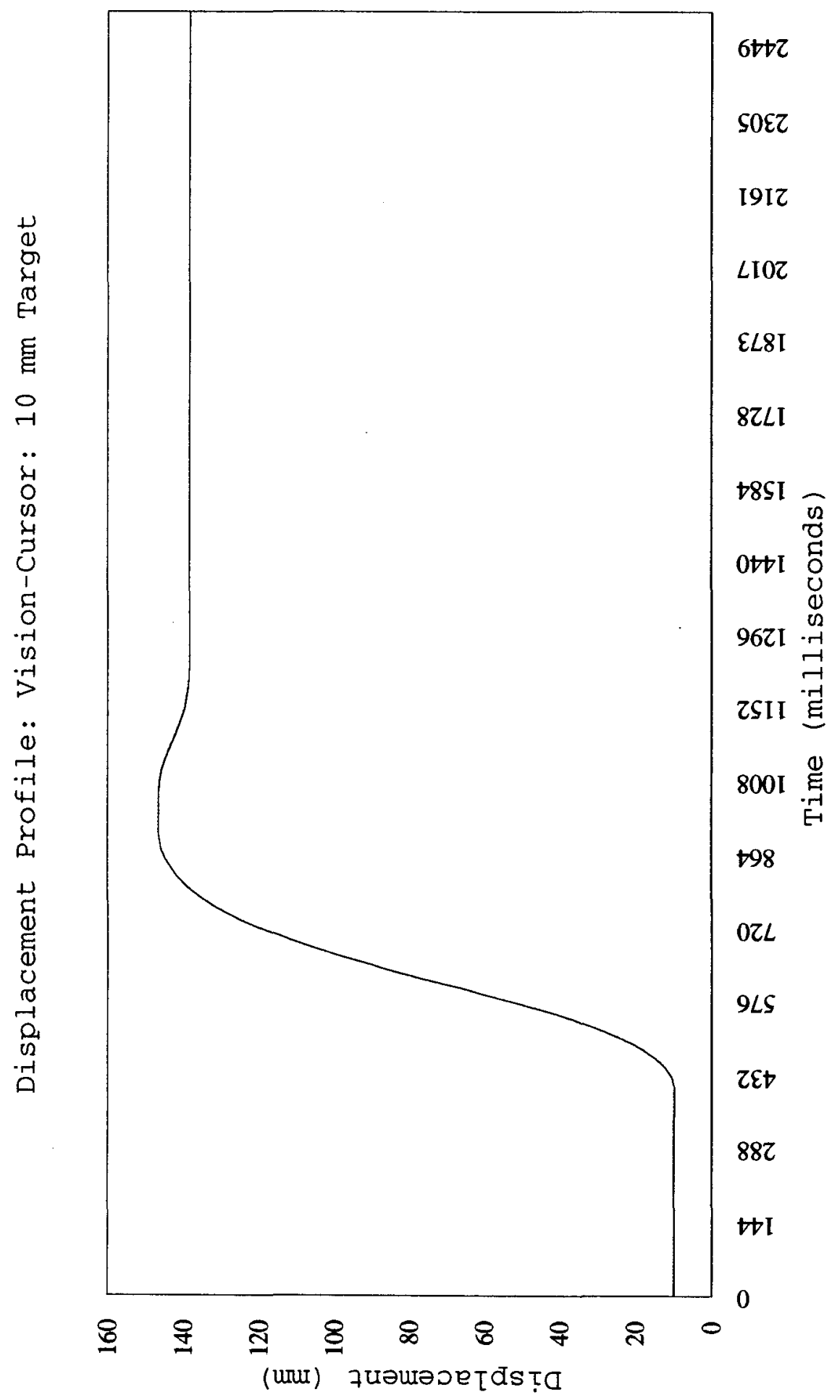




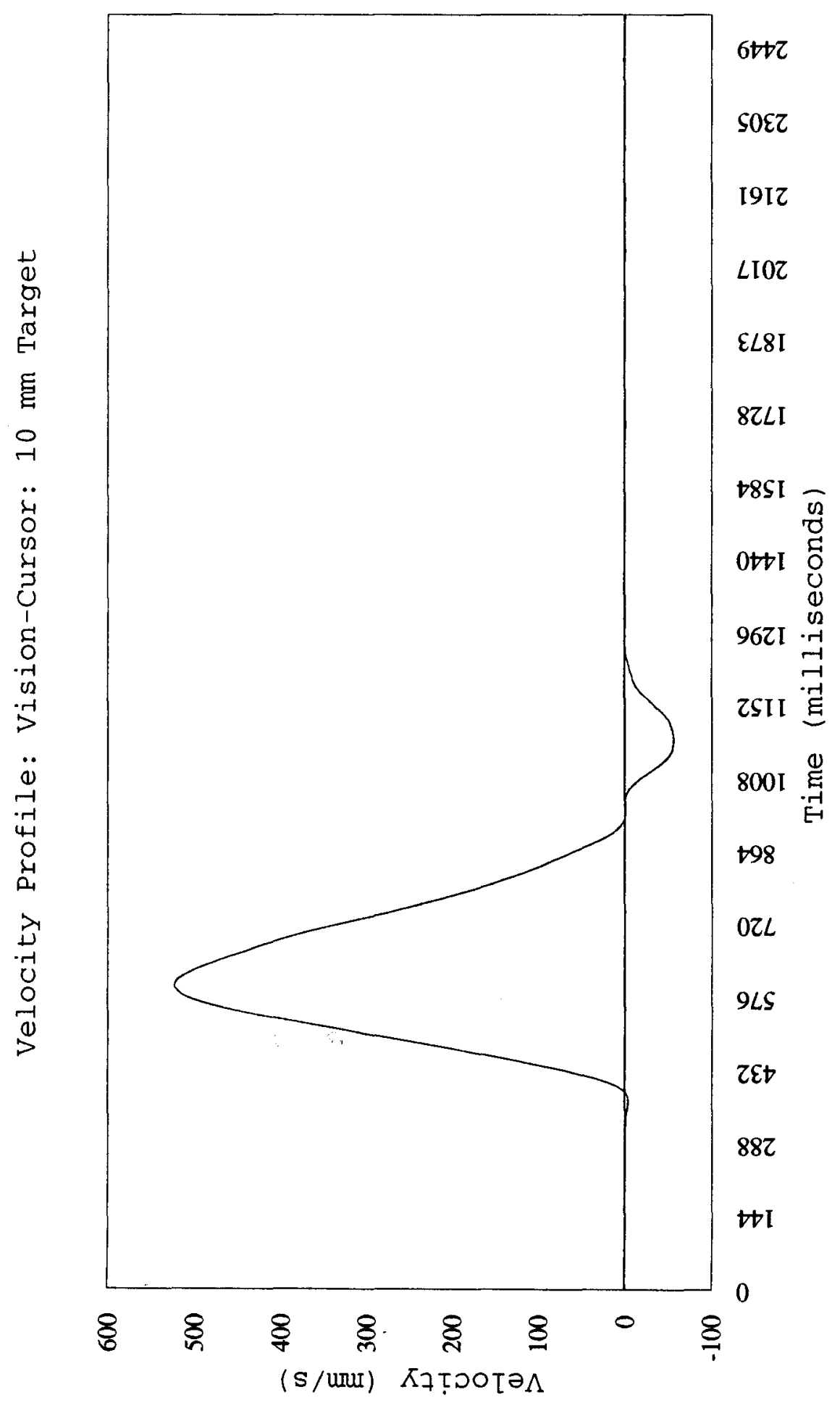




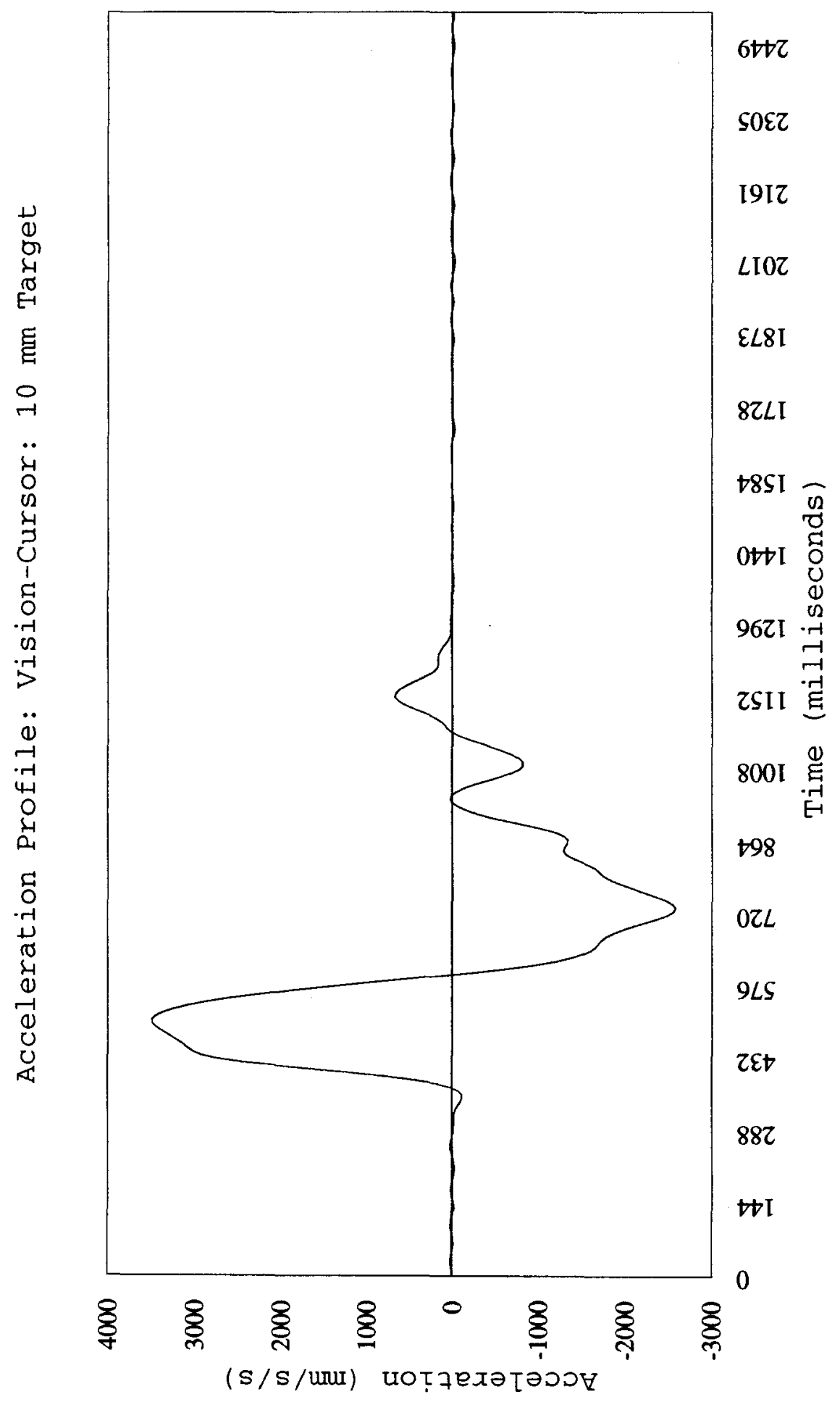


Appendix C

Statistical Analyses Summary Tables 
Table $1 \mathrm{a}$

Experiment 1: Analysis of Variance Summary Table for Movement Time

$\begin{array}{lccccc}\text { SOURCE } & \text { SS } & \text { DF } & \text { MS } & \text { F } & \text { P } \\ \text { Blocks/Subjects } & 500786.735 & 9 & & & \\ \text { Vision } & 194064.307 & 1 & 194064.307 & 10.596 & .009 \\ \text { Error } & 159417.609 & 9 & 17713.068 & & \\ \text { Target } & 39146.836 & 2 & 19573.418 & 3.499 & .051 \\ \text { Error } & 100702.672 & 18 & 5594.593 & & \\ \text { Vision Target } & 7531.650 & 2 & 3765.825 & .619 & \\ \text { Error } & 109560.609 & 18 & 6086.701 & & \\ \text { Total } & 1111210.420 & 59 & & & \\ \text { (Residual) } & 369680.891 & 45 & & & \end{array}$

Table 1b

Experiment 1: Movement Time Means and Standard Deviations

$\begin{array}{lcccc} & & 5 \mathrm{~mm} & 10 \mathrm{~mm} & 20 \mathrm{~mm} \\ \text { Vision-Cursor } & \overline{\mathrm{x}} & 821.3 & 813.23 & 744.5 \\ & \text { SD } & 178.0 & 153.8 & 85.1 \\ \text { No Vision-Cursor } & \overline{\mathrm{x}} & 705.2 & 673.3 & 659.3 \\ & \text { SD } & 129.3 & 94.5 & 92.2\end{array}$


Table $2 \mathrm{a}$

Experiment 1: Analysis of Variance summary Table for

Constant Error

$\begin{array}{lccccc}\text { SOURCE } & \text { SS } & \text { DF } & \text { MS } & \text { F } & \text { P } \\ \text { Blocks/Subjects } & 98.134 & 9 & & & \\ \text { Vision } & 28.954 & 1 & 28.954 & 3.439 & .094 \\ \text { Error } & 75.773 & 9 & 8.419 & & \\ \text { Target } & 14.213 & 2 & 7.107 & 1.134 & .344 \\ \text { Error } & 112.798 & 18 & 6.267 & & \\ \text { Vision Target } & 11.685 & 2 & 5.843 & & \\ \text { Error } & 117.867 & 18 & 6.548 & 0.892 & \\ \text { Total } & 447.739 & 59 & & & \\ \text { (Residual) } & 306.438 & 45 & & \end{array}$

Table 2b

Experiment 1: Constant Error Means and Standard Deviations

$5 \mathrm{~mm} \quad 10 \mathrm{~mm} \quad 20 \mathrm{~mm}$

Vision-Cursor

$\bar{x}$

0.06

$-0.02$

$-0.11$

$\mathrm{SD} \quad 0.5$

1.1

1.5

No Vision-Cursor

1.6

2.4

0.13

$\mathrm{SD} \quad 2.1$

3.9

4.5 
Table $3 a$

Experiment 1: Analysis of Variance Summary Table for

Variable Error

$\begin{array}{lccccc}\text { SOURCE } & \text { SS } & \text { DF } & \text { MS } & \text { F } & \text { P } \\ \text { Blocks/Subjects } & 66.309 & 9 & & & \\ \text { Vision } & 286.803 & 1 & 286.803 & 91.630 & <.001 \\ \text { Error } & 28.174 & 9 & 3.130 & & \\ \text { Target } & 4.648 & 2 & 2.324 & .572 \\ \text { Error } & 73.131 & 18 & 4.063 & & .025 \\ \text { Vision Target } & 30.717 & 2 & 15.358 & 4.530 & \\ \text { Error } & 61.023 & 18 & 3.390 & & \\ \text { Total } & 550.805 & 59 & & \end{array}$

Table 3b

Experiment 1: Variable Error Means and Standard Deviations

\begin{tabular}{|c|c|c|c|c|}
\hline & & $5 \mathrm{~mm}$ & $10 \mathrm{~mm}$ & $20 \mathrm{~mm}$ \\
\hline \multirow[t]{2}{*}{ Vision-Cursor } & $\bar{x}$ & 2.7 & 3.4 & 5.0 \\
\hline & SD & 1.6 & 1.8 & 1.8 \\
\hline No Vision-Cursor & $\bar{x}$ & 8.3 & 8.5 & 7.3 \\
\hline & SD & 2.3 & 2.6 & 2.2 \\
\hline
\end{tabular}


Table $4 a$

Experiment 1: Analysis of Variance Summary Table for Peak Velocity

\begin{tabular}{llllll} 
SOURCE & \multicolumn{1}{c}{ SS } & DF & MS & F & P \\
Blocks/Subjects & 227269.899 & 9 & & & \\
Vision & 9942.590 & 1 & 9942.590 & 2.612 & .138 \\
Error & 34256.504 & 9 & 3806.278 & & \\
Target & 3852.559 & 2 & 1926.279 & 2.042 & .157 \\
Error & 16980.184 & 18 & 943.344 & & \\
Vision Target & 195.417 & 2 & 97.709 & .081 \\
Error & 21791.010 & 18 & 1210.612 & & \\
Total & 314288.162 & 59 & & & \\
(Residual) & 73027.697 & 45 & &
\end{tabular}

Table $4 \mathrm{~b}$

Experiment 1: Peak Velocity Means and Standard Deviations

\begin{tabular}{lcccc} 
& & \multicolumn{1}{c}{$5 \mathrm{~mm}$} & $10 \mathrm{~mm}$ & $20 \mathrm{~mm}$ \\
Vision-Cursor & $\overline{\mathrm{x}}$ & 406.7 & 422.0 & 416.3 \\
& SD & 90.7 & 86.0 & 70.6 \\
No Vision-Cursor & $\overline{\mathrm{x}}$ & 375.9 & 399.0 & 392.8 \\
& SD & 79.7 & 67.8 & 42.6
\end{tabular}


Table $5 a$

Experiment 1: Analysis of Variance Summary Table for Time to Peak Velocity/Movement time

$\begin{array}{lccccc}\text { SOURCE } & \text { SS } & \text { DF } & \text { MS } & \text { F } & \text { P } \\ \text { Blocks/Subjects } & 182.209 & 9 & & & \\ \text { Vision } & 1938.130 & 1 & 1938.130 & 72.039 & <.001 \\ \text { Error } & 242.133 & 9 & 26.904 & & .815 \\ \text { Target } & 15.849 & 2 & 7.925 & & \\ \text { Error } & 175.028 & 18 & 9.724 & & \\ \text { Vision Target } & 38.123 & 2 & 19.061 & 1.349 & \\ \text { Error } & 254.243 & 18 & 14.125 & & \\ \text { Total } & 2845.715 & 59 & & & \\ \text { (Residual) } & 671.404 & 45 & & & \end{array}$

Table 5b

Experiment 1: Time to Peak Velocity/Movement time Means and Standard Deviations

\begin{tabular}{|c|c|c|c|c|}
\hline & & $5 \mathrm{~mm}$ & $10 \mathrm{~mm}$ & $20 \mathrm{~mm}$ \\
\hline \multirow[t]{2}{*}{ Vision-Cursor } & $\overline{\mathrm{x}}$ & 31.4 & 30.4 & 33.0 \\
\hline & SD & 3.7 & 4.7 & 3.7 \\
\hline No Vision-Cursor & $\overline{\mathrm{x}}$ & 42.0 & 44.0 & 42.9 \\
\hline & $\mathrm{SD}$ & 2.4 & 3.2 & 5.4 \\
\hline
\end{tabular}


Table $6 a$

Experiment 1: Analysis of Variance summary Table for

Displacement at Time of Peak Velocity/Target Distance

$\begin{array}{lccccc}\text { SOURCE } & \text { SS } & \text { DF } & \text { MS } & \text { F } & \text { P } \\ \text { Blocks/Subjects } & 196.647 & 9 & & & \\ \text { Vision } & 535.748 & 1 & 535.748 & 39.603 & <.001 \\ \text { Error } & 121.749 & 9 & 13.528 & & \\ \text { Target } & 24.997 & 2 & 12.499 & 2.219 & .136 \\ \text { Error } & 101.385 & 18 & 5.633 & & \\ \text { Vision Target } & 16.657 & 2 & 8.329 & .927 & \\ \text { Error } & 161.708 & 18 & 8.984 & & \\ \text { Total } & 1158.892 & 59 & & & \\ \text { (Residual) } & 384.843 & 45 & & \end{array}$

Table $6 \mathrm{~b}$

Experiment 1: Displacement at Time of Peak Velocity/Target

Distance Means and Standard Deviations

\begin{tabular}{|c|c|c|c|c|}
\hline & & $5 \mathrm{~mm}$ & $10 \mathrm{~mm}$ & $20 \mathrm{~mm}$ \\
\hline \multirow[t]{2}{*}{ Vision-Cursor } & $\bar{x}$ & 40.8 & 41.2 & 41.0 \\
\hline & SD & 2.8 & 3.6 & 3.1 \\
\hline \multirow[t]{2}{*}{ No Vision-Cursor } & $\bar{x}$ & 46.1 & 48.7 & 46.2 \\
\hline & $S D$ & 3.1 & 2.4 & 4.4 \\
\hline
\end{tabular}


Table $7 a$

Experiment 1: Analysis of Variance Summary Table for Total Number of Significant Deviations in the Acceleration Profile Following Peak Velocity

$\begin{array}{lccccc}\text { SOURCE } & \text { SS } & \text { DF } & \text { MS } & \text { F } & \text { P } \\ \text { Blocks/Subjects } & 1368.933 & 9 & & & \\ \text { Vision } & 308.267 & 1 & 308.267 & 7.174 & .024 \\ \text { Error } & 386.733 & 9 & 42.970 & & \\ \text { Target } & 122.233 & 2 & 61.117 & 2.951 & .076 \\ \text { Error } & 372.767 & 18 & 20.709 & & \\ \text { Vision Target } & 57.633 & 2 & 28.817 & 1.756 & .200 \\ \text { Error } & 295.367 & 18 & 16.409 & & \\ \text { Total } & 2911.933 & 59 & & & \\ \text { (Residual) } & 1054.867 & 45 & & & \end{array}$

Table $7 \mathrm{~b}$

Experiment 1: PVSD Mean Sums and Standard Deviations

$\begin{array}{lcccc} & & 5 \mathrm{~mm} & 10 \mathrm{~mm} & 20 \mathrm{~mm} \\ \text { Vision-Cursor } & \overline{\mathrm{x}} & 12.0 & 10.6 & 11.1 \\ & \text { SD } & 7.7 & 5.7 & 7.2 \\ \text { No Vision-Cursor } & \overline{\mathrm{x}} & 9.9 & 6.0 & 4.2 \\ & \text { SD } & 8.4 & 6.3 & 4.1\end{array}$


Table 8

Experiment 1: Friedman Analysis of Variance Summary Table for Total Number of zero crossings in the Acceleration Profile Following Peak Velocity

$\begin{array}{ll}\text { DEGREES FREEDOM } & 5 \\ \text { \# SAMPLES } & 6 \\ \text { \# CASES } & 10 \\ \text { CHIr-SQUARED } & 31.886\end{array}$

CONDITION

NC $5 \mathrm{~mm}$

NC $10 \mathrm{~mm}$

NC $20 \mathrm{~mm}$

VC $5 \mathrm{~mm}$

VC $10 \mathrm{~mm}$

VC $20 \mathrm{~mm}$
$\Sigma$ RANK

21.5

19.5

24.5

49.5

53

42
MEAN RANK

2.15

1.95

2.45

4.95

5.3

4.2
MEAN SUM

0.4

0.1

0.5

4.5

6.1

2.8 
Table 9

Experiment 1: Friedman Analysis of Variance Summary Table for the Number of Zero crossings in the Acceleration profile Following Peak Velocity - Test for Effect of Target size

$\begin{array}{llc}\text { DEGREES FREEDOM } & 2 \\ \text { \# SAMPLES } & 3 & \\ \text { \# CASES } & 10 & \\ \text { CHIr-SQUARED } & 2.85 & \\ & & \\ \text { CONDITION } & \sum \text { RANK } & 2.05 \\ 5 \mathrm{~mm} & 20.5 & 2.35 \\ 10 \mathrm{~mm} & 23.5 & 1.6 \\ 20 \mathrm{~mm} & 16 & \end{array}$


Table 10

Experiment 1: Friedman Analysis of Variance Summary Table for the Number of zero crossings in the Acceleration profile Following Peak Velocity - Test for Effect of Vision Condition

DEGREES FREEDOM

\# SAMPLES

\# CASES

CHIr-SQUARED

CONDITION

Vision-Cursor

No Vision-Cursor
1

2

10

10.000

$\sum$ RANK

20

10
MEAN RANK

2.0

1.0 
Table 11

Experiment 1: Friedman Analysis of Variance Summary Table for Total Number of Significant Deviations in the Acceleration profile prior to Peak Velocity

$\begin{array}{ll}\text { DEGREES FREEDOM } & 5 \\ \# \text { SAMPLES } & 6 \\ \# \text { CASES } & 10 \\ \text { CHIr-SQUARED } & 10.143\end{array}$

CONDITION

NC $5 \mathrm{~mm}$

NC $10 \mathrm{~mm}$

NC $20 \mathrm{~mm}$

VC $5 \mathrm{~mm}$

VC $10 \mathrm{~mm}$

VC $20 \mathrm{~mm}$ $\sum$ RANK

44

46.5

30.5

34

29.5

25.5
MEAN RANK

4.4

4.65

3.05

3.4

2.95

2.55
MEAN SUM

3.9

3.7

1.8

2.2

1.9

1. 4 
Table 12

Experiment 1: Friedman Analysis of Variance Summary Table

for Reversals in Movement Direction

$\begin{array}{ll}\text { DEGREES FREEDOM } & 5 \\ \text { \# SAMPLES } & 6 \\ \text { \# CASES } & 10 \\ \text { CHIr-SQUARED } & 2.771\end{array}$

CONDITION

NC $5 \mathrm{~mm}$

NC $10 \mathrm{~mm}$

NC $20 \mathrm{~mm}$

VC $5 \mathrm{~mm}$

VC $10 \mathrm{~mm}$

VC $20 \mathrm{~mm}$ $\sum$ RANK

30.5

32.5

31.5

35.5

42

38
MEAN RANK

3.05

3.25

3.15

3.55

4.2

- 3.8
MEAN SUM

0.5

0.6

0.8

0.6

1.1

1.0 
Table $13 a$

Experiment 1: Analysis of Variance Summary Table for

Movement Time and Total Number of Modifications

Correlation/Fisher Z-scores

$\begin{array}{lccccc}\text { SOURCE } & \text { SS } & \text { DF } & \text { MS } & \text { F } & \text { P } \\ \text { Blocks/Subjects } & 1.490 & 9 & & & \\ \text { Vision } & 1.637 & 1 & 1.637 & 5.724 & .038 \\ \text { Error } & 2.575 & 9 & .286 & & \\ \text { Target } & .391 & 2 & .195 & 1.639 & .221 \\ \text { Error } & 2.149 & 18 & .119 & & \\ \text { Vision Target } & .170 & 2 & .085 & .649 & \\ \text { Error } & 2.367 & 18 & .131 & & \\ \text { Total } & 10.778 & 59 & & & \\ \text { (Residual) } & 7.091 & 45 & & \end{array}$

Table $13 \mathrm{~b}$

Experiment 1: Fisher $\mathrm{Z}$-score Means and Standard Deviations

$\begin{array}{lllll} & & 5 \mathrm{~mm} & 10 \mathrm{~mm} & 20 \mathrm{~mm} \\ \text { Vision-Cursor } & \overline{\mathrm{x}} & 1.04 & 1.01 & 0.97 \\ & \mathrm{SD} & 0.34 & 0.53 & 0.31 \\ \text { No Vision-Cursor } & \overline{\mathrm{x}} & 0.86 & 0.61 & 0.56 \\ & \mathrm{SD} & 0.34 & 0.38 & 0.45\end{array}$


Table $14 a$

Experiment 1: Analysis of Variance Summary Table for Time

from Peak Velocity to End of Movement and Absolute Error

correlation/Fisher $\mathrm{Z}$-scores

$\begin{array}{lccccc}\text { SOURCE } & \text { SS } & \text { DF } & \text { MS } & \text { F } & \text { P } \\ \text { Blocks/Subjects } & 1.638 & 9 & & & \\ \text { Vision } & 2.460 & 1 & 2.460 & 10.885 & .009 \\ \text { Error } & 2.034 & 9 & .226 & & .029 \\ \text { Target } & 1.317 & 2 & .659 & 4.307 & \\ \text { Error } & 2.760 & 18 & .153 & & \\ \text { Vision Target } & .067 & 2 & .033 & .260 & \\ \text { Error } & 2.288 & 18 & .127 & & \\ \text { Total } & 12.565 & 59 & & \\ \text { (Residual) } & 7.083 & 45 & & \end{array}$

Table $14 \mathrm{~b}$

Experiment 1: Fisher $\mathrm{Z}$-score Means and Standard Deviations

$\begin{array}{lcccc} & & 5 \mathrm{~mm} & 10 \mathrm{~mm} & 20 \mathrm{~mm} \\ \text { Vision-Cursor } & \overline{\mathrm{x}} & -0.54 & -0.42 & -0.21 \\ & \text { SD } & 0.39 & 0.36 & 0.34 \\ \text { No Vision-Cursor } & \overline{\mathrm{x}} & -0.21 & 0.07 & 0.19 \\ & \text { SD } & 0.25 & 0.47 & 0.53\end{array}$


Table $15 a$

Experiment 1: Analysis of Variance Summary Table for Total number of Modifications and Absolute Error Correlation/Fisher $\underline{\text { Z-scores }}$

\begin{tabular}{|c|c|c|c|c|c|}
\hline SOURCE & SS & $\mathrm{DF}$ & MS & $\mathrm{F}$ & $\mathrm{P}$ \\
\hline Blocks/Subjects & 1875 & 9 & & & \\
\hline Vision & 647 & 1 & 647 & 2557 & 142 \\
\hline Error & 2280 & 9 & .253 & & \\
\hline Target & 772 & 2 & 386 & 1643 & 220 \\
\hline Error & 4223 & 18 & 235 & & \\
\hline Vision Target & 206 & 2 & 103 & 512 & \\
\hline Error & 3621 & 18 & 201 & & \\
\hline Total & $13 \quad 623$ & 59 & & & \\
\hline (Residual) & & 45 & & & \\
\hline
\end{tabular}

Table 15b

Experiment 1: Fisher $\mathrm{Z}$-score Means and Standard Deviations

\begin{tabular}{|c|c|c|c|c|}
\hline & & $5 \mathrm{~mm}$ & $10 \mathrm{~mm}$ & $20 \mathrm{~mm}$ \\
\hline \multirow[t]{2}{*}{ Vision-Cursor } & $\bar{x}$ & -046 & -039 & $\begin{array}{ll}-0 & 07\end{array}$ \\
\hline & SD & 029 & 046 & 049 \\
\hline \multirow[t]{2}{*}{ No Vision-Cursor } & $\bar{x}$ & -019 & $\begin{array}{ll}-0 & 09\end{array}$ & $\begin{array}{ll}-0 & 03\end{array}$ \\
\hline & SD & $\begin{array}{ll}0 & 47\end{array}$ & 049 & $0 \quad 58$ \\
\hline
\end{tabular}


Table $16 a$

Experiment 2: Analysis of Variance Summary Table for

Movement Time

$\begin{array}{lccccc}\text { SOURCE } & \text { SS } & \text { DF } & \text { MS } & \text { F } & \text { P } \\ \text { Blocks/Subjects } & 1719509.600 & 9 & & & \\ \text { Vision } & 61051.563 & 5 & 12210.313 & 1.060 & .395 \\ \text { Error } & 518595.305 & 45 & 11524.340 & \\ \text { Total } & 2299156.470 & 59 & & \end{array}$

Table 16b

Experiment 2: Movement Time Means and Standard Deviations

$\begin{array}{rllllll}\text { Condition } & \text { VC } & \text { VXFH } & \text { VXLH } & \text { NV } & \text { NXFH } & \text { NXLH } \\ \overline{\mathrm{x}} & 618.0 & 631.2 & 658.5 & 555.9 & 593.2 & 618.2 \\ \text { SD } & 218.8 & 198.1 & 269.4 & 167.1 & 160.8 & 187.5\end{array}$


Table $17 \mathrm{a}$

Experiment 2: Analysis of Variance Summary Table for

Constant Error

$\begin{array}{lccccc}\text { SOURCE } & \text { SS } & \text { DF } & \text { MS } & \text { F } & \text { P } \\ \text { Blocks/Subjects } & 32.162 & 9 & & & \\ \text { Vision } & 191.554 & 5 & 38.311 & 7.124 & <.001 \\ \text { Error } & 242.011 & 45 & 5.378 & & \\ \text { Total } & 465.727 & 59 & & \end{array}$

Table $17 \mathrm{~b}$

Experiment 2: Constant Error Means and Standard Deviations

$\begin{array}{rcccccc}\text { Condition } & \text { VC } & \text { VXFH } & \text { VXLH } & \text { NV } & \text { NXFH } & \text { NXLH } \\ \overline{\mathrm{x}} & 0.2 & 2.8 & -1.1 & -1.2 & 3.5 & 0.6 \\ \text { SD } & 1.9 & 3.0 & 1.4 & 2.6 & 2.5 & 1.8\end{array}$


Table 18a

Experiment 2: Analysis of Variance Summary Table for

Variable Error

$\begin{array}{lccccc}\text { SOURCE } & \text { SS } & \text { DF } & \text { MS } & \text { F } & \text { P } \\ \text { Blocks/Subjects } & 95.701 & 9 & & & \\ \text { Vision } & 110.369 & 5 & 22.074 & 9.665 & <.001 \\ \text { Error } & 102.787 & 45 & 2.284 & & \\ \text { Total } & 308.856 & 59 & & \end{array}$

Table $18 a$

Experiment 2: Variable Error Means and standard Deviations

$\begin{array}{llllll}\text { Condition } & \text { VC } & \text { VXFH } & \text { VXLH } & \text { NV } & \text { NXFH }\end{array}$

$\begin{array}{lllllll}\overline{\mathrm{x}} & 4.6 & 6.7 & 4.7 & 8.3 & 7.4 & 5.6 \\ \mathrm{SD} & 2.4 & 1.4 & 2.0 & 2.0 & 1.8 & 1.8\end{array}$


Table 19a

Experiment 2: Analysis of Variance Summary Table for Peak Velocity

\begin{tabular}{|c|c|c|c|c|}
\hline SOURCE & SS & $\mathrm{DF}$ & MS & $\mathrm{F}$ \\
\hline Blocks/Subjects & 2048534.660 & 9 & & \\
\hline Vision & 2385.698 & 5 & 477.140 & .081 \\
\hline Error & 265896.289 & 45 & 5908.806 & \\
\hline Total & 2316816.640 & 59 & & \\
\hline
\end{tabular}

Table $19 \mathrm{~b}$

Experiment 2: Peak Velocity Means and Standard Deviations

$\begin{array}{rllllll}\text { Condition } & \text { VC } & \text { VXFH } & \text { VXLH } & \text { NV } & \text { NXFH } & \text { NXLH } \\ \overline{\mathrm{x}} & 532.1 & 529.3 & 529.9 & 544.2 & 541.5 & 542.8 \\ \text { SD } & 179.1 & 167.2 & 181.4 & 237.2 & 227.7 & 236.8\end{array}$


Table 20a

Experiment 2: Analysis of Variance Summary Table for Time to Peak Velocity/Movement time

$\begin{array}{lccccc}\text { SOURCE } & \text { SS } & \text { DF } & \text { MS } & \text { F } & \text { P } \\ \text { Blocks/Subjects } & 624.721 & 9 & & & \\ \text { Vision } & 365.969 & 5 & 73.194 & 3.622 & .007 \\ \text { Error } & 909.289 & 45 & 20.206 & & \\ \text { Total } & 1899.980 & 59 & & \end{array}$

Table $20 \mathrm{~b}$

Experiment 2: Peak Velocity/Movement time Means and standard Deviations

$\begin{array}{rllllll}\text { Condition } & \text { VC } & \text { VXFH } & \text { VXLH } & \text { NV } & \text { NXFH } & \text { NXLH } \\ \overline{\mathrm{x}} & 35.5 & 34.2 & 34.8 & 41.3 & 38.4 & 38.0 \\ \text { SD } & 5.2 & 5.0 & 6.7 & 4.9 & 4.6 & 5.5\end{array}$


Table $21 a$

Experiment 2: Analysis of Variance summary Table for

Displacement at Time of Peak Velocity/Target Distance

$\begin{array}{lccccc}\text { SOURCE } & \text { SS } & \text { DF } & \text { MS } & \text { F } & \text { P } \\ \text { Blocks/Subjects } & 520.826 & 9 & & & \\ \text { Vision } & 197.519 & 5 & 39.504 & 4.250 & .003 \\ \text { Error } & 418.285 & 45 & 9.295 & \\ \text { Total } & 1136.631 & 59 & & \end{array}$

Table $21 b$

Experiment 2: Displacement at Time of Peak Velocity/Target Distance Means and Standard Deviations

$\begin{array}{rllllll}\text { Condition } & \text { VC } & \text { VXFH } & \text { VXLH } & \text { NV } & \text { NXFH } & \text { NXLH } \\ \overline{\mathrm{x}} & 42.3 & 42.0 & 42.5 & 45.9 & 45.9 & 45.7 \\ \text { SD } & 4.5 & 4.8 & 5.4 & 3.1 & 3.3 & 3.4\end{array}$


Table 22

Experiment 2: Friedman Analysis of Variance Summary Table for the Mean Number of Significant Deviations in the Acceleration Profile prior to Peak Velocity

\begin{tabular}{lll} 
DEGREES FREEDOM & \multicolumn{1}{l}{} \\
\# SAMPLES & 6 & \\
\# CASES & 10 & \\
CHIY-SQUARED & 1.571 & MEAN RANK \\
& & 3.5 \\
CONDITION & I RANK & 3.1 \\
VC & 35 & 3.15 \\
VXFH & 31 & 3.75 \\
VXLH & 31.5 & 3.55 \\
NV & 37.5 & 3.95 \\
NXFH & 35.5 & \\
NXLH & 39.5 &
\end{tabular}


Table 23

Experiment 2: Friedman Analysis of Variance Summary Table for the Mean Number of Significant Deviations in the Acceleration Profile Following Peak Velocity

$\begin{array}{lll}\text { DEGREES FREEDOM } & 5 \\ \text { \# SAMPLES } & 6 & \\ \text { \# CASES } & 10 & \\ \text { CHIr-SQUARED } & 5.157 & \\ & & \text { MEAN RANK } \\ \text { CONDITION } & \text { L RANK } & 3.3 \\ \text { VC } & 33 & 4.5 \\ \text { VXFH } & 45 & 3.3 \\ \text { VXLH } & 33 & 2.75 \\ \text { NV } & 27.5 & 3.85 \\ \text { NXFH } & 38.5 & 3.3 \\ \text { NXLH } & 33 & \end{array}$




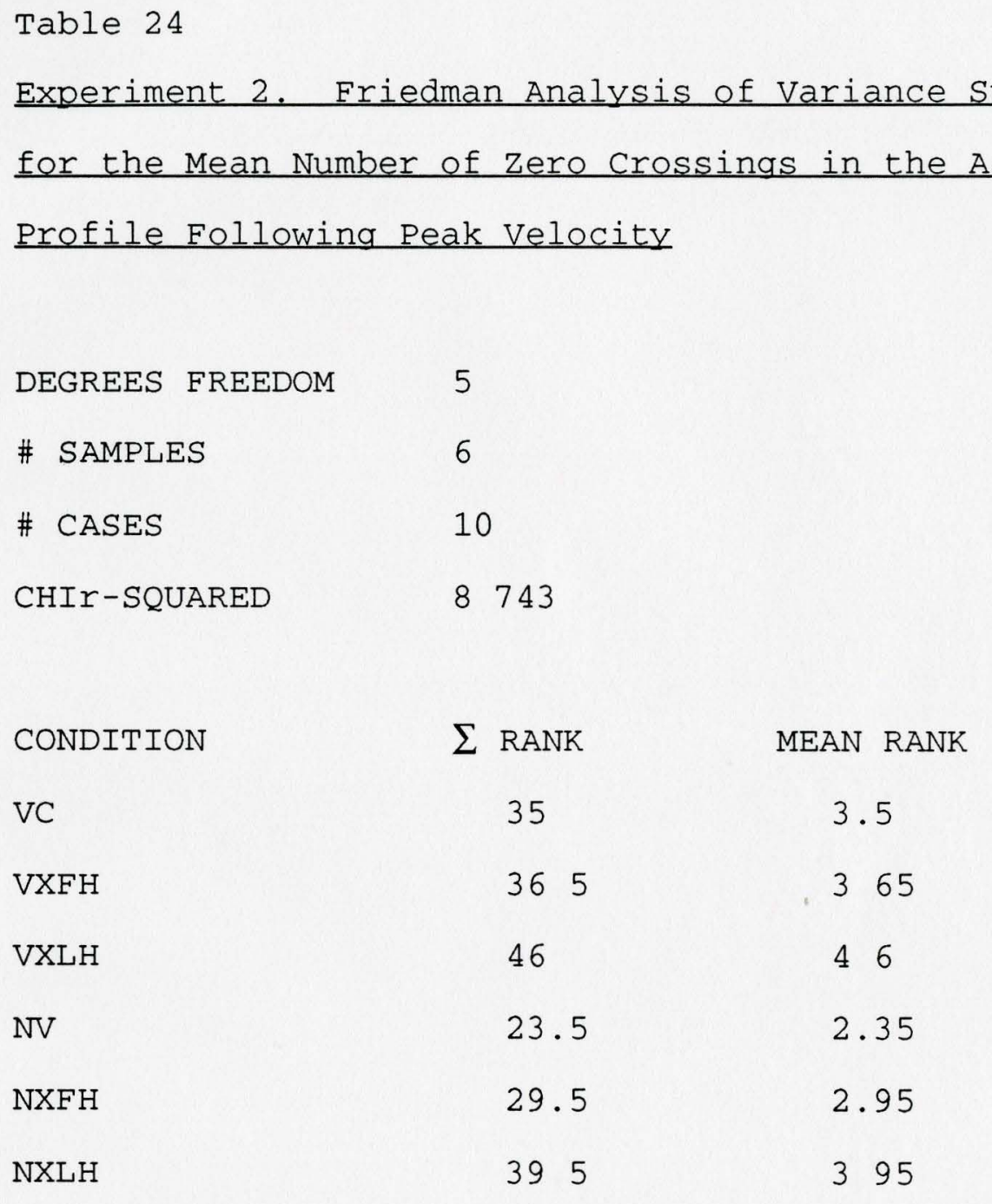


Table 25

Experiment 2: Friedman Analysis of Variance Summary Table

for Mean Number of Reversals in Movement Direction

DEGREES FREEDOM 5

\# SAMPLES

\# CASES

CHIr-SQUARED

CONDITION

VC

VXFH

VXLH

NV

NXFH

NXLH
5

6

10

2514

$\sum$ RANK

415

345

37.5

32

29.5

35
MEAN RANK

4.15

345

375

32

295

35 
Table 26

Experiment 2. Movement Modification Means Collapsed Across

Subjects

Movement Modification

Condition

SDPV

PVSD

$\mathrm{ZC}$

REVS

VC

008

053

0 19

013

VXFH

$\begin{array}{ll}0 & 07\end{array}$

0.68

0.19

$\begin{array}{ll}0 & 08\end{array}$

VXLH

0.07

056

0.39

$\begin{array}{ll}0 & 12\end{array}$

NV

010

028

0.06

0 11

$\mathrm{NXFH}$

$\begin{array}{ll}0 & 13\end{array}$

046

$\begin{array}{ll}0 & 14\end{array}$

0 10

NXLH

0.13

$0 \quad 35$

0.23

013 
Table $27 a$

Experiment 2: Analysis of Variance Summary Table for

Movement Time and Total Number of Modifications

Correlation/Fisher Z-scores

\begin{tabular}{|c|c|c|c|c|c|}
\hline SOURCE & SS & $\mathrm{DF}$ & MS & $\mathrm{F}$ & $\mathrm{P}$ \\
\hline Blocks/Subjects & 4105 & 9 & & & \\
\hline Vision & 2280 & 5 & 456 & 3020 & 01 \\
\hline Error & 6784 & 45 & 151 & & \\
\hline Total & 13169 & 59 & & & \\
\hline
\end{tabular}

Table 27b

Experiment 2. Fisher $\mathrm{Z}$-score Means and Standard Deviations

$\begin{array}{clllllll}\text { Condition } & \text { VC } & \text { VXFH } & \text { VXLH } & \text { NV } & \text { NXFH } & \text { NXLH } \\ \bar{x} & 091 & 098 & 090 & 040 & 068 & 070 \\ \text { SD } & 044 & 035 & 055 & 036 & 047 & 050\end{array}$


Table $28 a$

Experiment 2: Analysis of Variance Summary Table for Time from Peak Velocity to End of Movement and Absolute Error Correlation/Fisher Z-scores

\begin{tabular}{|c|c|c|c|c|c|}
\hline SOURCE & SS & DF & MS & & $F$ \\
\hline Blocks/Subjects & 1183 & 9 & & & \\
\hline Vision & 1990 & 5 & 398 & 3 & 827 \\
\hline Error & 4683 & 45 & 104 & & \\
\hline Total & 7857 & 59 & & & \\
\hline
\end{tabular}

Table $28 \mathrm{~b}$

Experiment 2: Fisher $\mathrm{Z}$-score Means and Standard Deviations

\begin{tabular}{|c|c|c|c|c|c|c|}
\hline Condition & VC & VXFH & VXLH & NV & $\mathrm{NXFH}$ & $\mathrm{NXLH}$ \\
\hline$\overline{\mathrm{x}}$ & -026 & 014 & $\begin{array}{ll}-0 & 41\end{array}$ & -006 & -024 & $\begin{array}{ll}-0 & 31\end{array}$ \\
\hline SD & 029 & 035 & 031 & 027 & 038 & 036 \\
\hline
\end{tabular}


Table 29 a

Experiment 2: Analysis of Variance Summary Table for Total

Number of Modifications and Absolute Error Correlation/Fisher

$\underline{z \text {-scores }}$

\begin{tabular}{|c|c|c|c|c|c|c|}
\hline SOURCE & SS & $\mathrm{DF}$ & MS & & $F$ & $P$ \\
\hline Blocks/Subjects & 756 & 9 & & & & \\
\hline Vision & 1153 & 5 & 231 & 3 & 122 & 016 \\
\hline Error & 3342 & 45 & 074 & & & \\
\hline Total & 5251 & 59 & & & & \\
\hline
\end{tabular}

Table $29 b$

Experiment 2: Fisher $\mathrm{Z}$-score Means and Standard Deviations

\begin{tabular}{|c|c|c|c|c|c|c|}
\hline condition & VC & VXFH & VXLH & NV & $\mathrm{NXFH}$ & NXLH \\
\hline $\bar{x}$ & -016 & 005 & -038 & $\begin{array}{ll}-0 & 02\end{array}$ & $-0 \quad 09$ & $\begin{array}{ll}-0 & 21\end{array}$ \\
\hline SD & 027 & 0.37 & 025 & 013 & 029 & 028 \\
\hline
\end{tabular}


Table 30a

Experiment 2 Partial Vision Analysis: Analysis of Variance Summary Table for Movement Time

\begin{tabular}{|c|c|c|c|c|c|}
\hline SOURCE & SS & DF & MS & $\mathrm{F}$ & $\mathrm{P}$ \\
\hline Blocks/Subjects & $1972248 \quad 800$ & 9 & & & \\
\hline Partialvision & 33353916 & 1 & 33353916 & 5739 & 038 \\
\hline Error & $52310 \quad 313$ & 9 & $5812 \quad 257$ & & \\
\hline MixType & $24543 \quad 447$ & 2 & 12271724 & 533 & \\
\hline Error & 414226789 & 18 & 23012599 & & \\
\hline \multicolumn{6}{|l|}{ Partialvision } \\
\hline x Mixtype & $13203 \quad 174$ & 2 & 6601587 & 2723 & .091 \\
\hline Error & 43632861 & 18 & $2424 \quad 048$ & & \\
\hline Total & 2553519260 & 59 & & & \\
\hline (Residual) & 510169963 & 45 & & & \\
\hline
\end{tabular}

Table $30 b$

Experiment 2 Partial Vision Analysis: Movement Time Means and Standard Deviations

Vision Mix No Vision Mix Blocked

$\begin{array}{cccccc}\text { First-Half Vision } & \overline{\mathrm{x}} & 6312 & 5932 & 5544 \\ & \text { SD } & 198 & 1 & 160 & 1817 \\ \text { Last-Half Vision } & \overline{\mathrm{x}} & 658 & 5 & 6182 & 6435 \\ & \text { SD } & 2694 & 187 & 2645\end{array}$


Table $31 a$

Experiment 2 partial Vision Analysis. Analysis of Variance

Summary Table for Constant Error

\begin{tabular}{|c|c|c|c|c|c|}
\hline SOURCE & SS & DF & MS & $\mathrm{F}$ & $\mathrm{P}$ \\
\hline Blocks/Subjects & 42868 & 9 & & & \\
\hline Partialvision & 118807 & 1 & $118 \quad 807$ & $32 \quad 127$ & $<001$ \\
\hline Error & 33279 & 9 & 3698 & & \\
\hline MixType & $27 \quad 125$ & 2 & 13562 & 3829 & 040 \\
\hline Error & 63759 & 18 & 3542 & & \\
\hline \multicolumn{6}{|l|}{ Partialvision } \\
\hline x Mixtype & $13 \quad 022$ & 2 & 6511 & 1377 & 277 \\
\hline Error & 85093 & 18 & 4727 & & \\
\hline Total & 383952 & 59 & & & \\
\hline (Residual) & $182 \quad 131$ & 45 & & & \\
\hline
\end{tabular}

Table $31 b$

Experiment 2 Partial Vision Analysis. Constant Error Means and Standard Deviations

Vision Mix No Vision Mix Blocked

$\begin{array}{cccccc}\text { First-Half Vision } & \overline{\mathrm{x}} & 28 & 35 & 13 \\ & \text { SD } & 30 & 25 & 19 \\ \text { Last-Half Vision } & \overline{\mathrm{x}} & -11 & 0 & -03 \\ & \text { SD } & 14 & 18 & 10\end{array}$


Table 32 a

Experiment 2 Partial Vision Analysis: Analysis of Variance Summary Table for Variable Error

\begin{tabular}{|c|c|c|c|c|c|}
\hline SOURCE & SS & DF & MS & $\mathrm{F}$ & $\mathrm{P}$ \\
\hline Blocks/Subjects & 91693 & 9 & & & \\
\hline PartialVision & $59 \quad 541$ & 1 & $59 \quad 541$ & 26700 & $<001$ \\
\hline Error & $20 \quad 073$ & 9 & 2230 & & \\
\hline MixType & 6549 & 2 & 3275 & 908 & \\
\hline Error & 64951 & 18 & 3608 & & \\
\hline \multicolumn{6}{|l|}{ Partialvision } \\
\hline x Mixtype & 478 & 2 & 239 & 161 & \\
\hline Error & 26720 & 18 & 1484 & & \\
\hline Total & $270 \quad 006$ & 59 & & & \\
\hline (Residual) & 111744 & 45 & & & \\
\hline
\end{tabular}

Table $32 b$

Experiment 2 Partial Vision Analysis: Variable Error Means and Standard Deviations

Vision Mix No Vision Mix Blocked

$\begin{array}{lllll}\text { First-Half Vision } & \overline{\mathrm{x}} & 67 & 74 & 73 \\ & \mathrm{SD} & 14 & 18 & 20 \\ \text { Last-Half Vision } & \overline{\mathrm{x}} & 47 & 56 & 50 \\ & \text { SD } & 20 & 18 & 25\end{array}$


Table $33 a$

Experiment 2 Partial Vision Analysis: Analysis of Variance Summary Table for Peak Velocity

SOURCE

Blocks/Subjects

Partialvision

Error

MixType

Error

PartialVision

$$
\text { x Mixtype }
$$

Error

Total

(Residual)
SS

1988921610

315840

3234742

8410923

240992516

911777

$12308 \quad 591$

2255095990

256535849
DF

MS

9

1

9

2

18

315840

879

359416

$4205 \quad 461$

314

$13388 \quad 473$

2

455889

667

$18 \quad 683 \quad 811$

59

45

Table 33b

Experiment 2 Partial Vision Analysis: Peak Velocity Means and Standard Deviations

Vision Mix

$\overline{\mathrm{x}}$

First-Half Vision

Last-Half Vision

5293

1672

5299

$\bar{x}$

SD

20

1814

SD

SD

1814

No Vision Mix

Blocked

$566 \quad 4$
$207 \quad 5$
5508
$194 \quad 0$


Table $34 a$

Experiment 2 partial Vision Analysis: Analysis of Variance Summary Table for Time to Peak Velocity/Movement Time

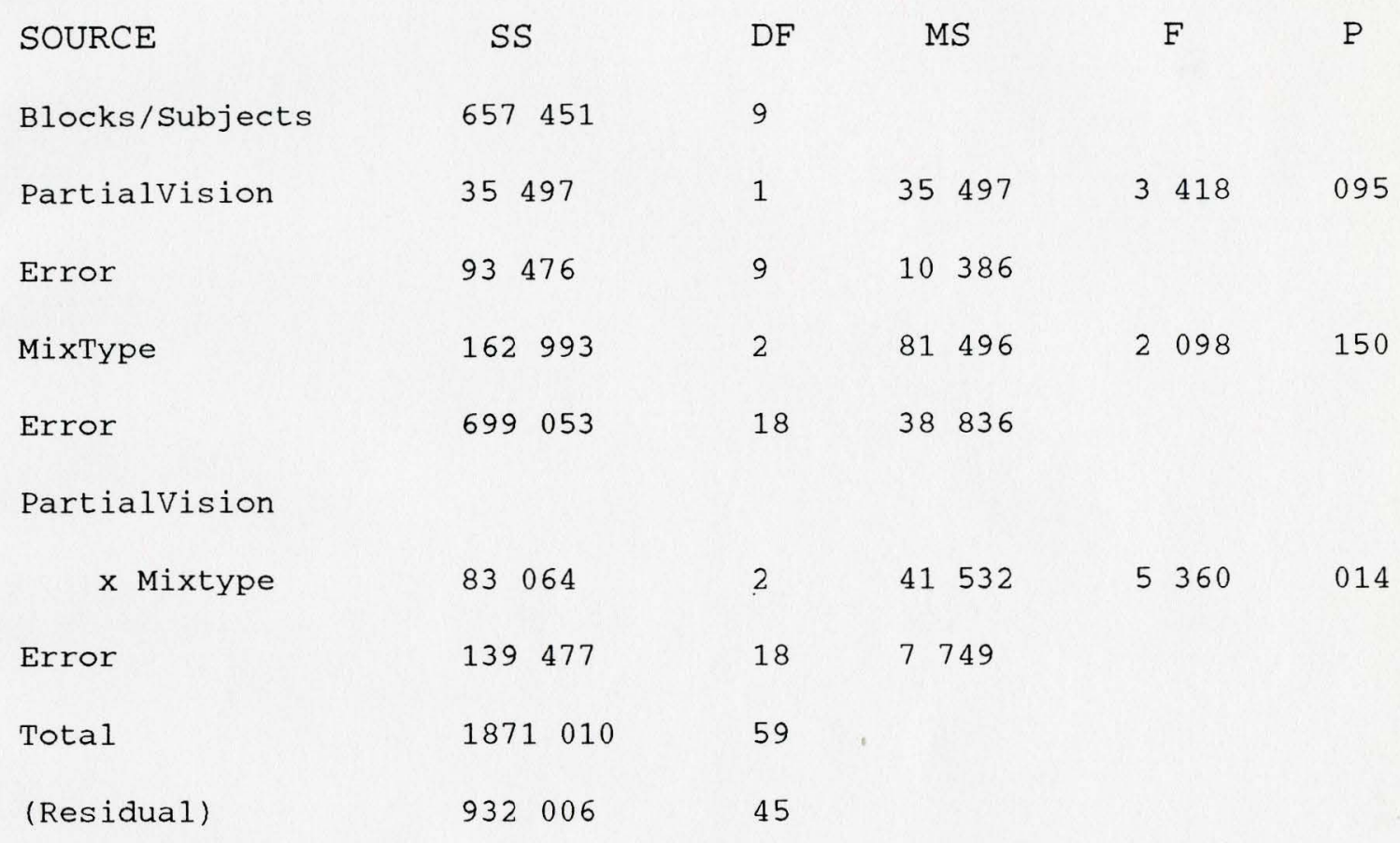

Table $34 b$

Experiment 2 Partial Vision Analysis. Time to Peak

Velocity/Movement Time Means and Standard Deviations

Vision Mix No Vision Mix Blocked

$\begin{array}{lllll}\text { First-Half Vision } & \overline{\mathrm{x}} & 342 & 384 & 400 \\ & \text { SD } & 50 & 46 & 51 \\ \text { Last-Half Vision } & \overline{\mathrm{x}} & 348 & 380 & 352 \\ & \text { SD } & 67 & 55 & 55\end{array}$


Table $35 a$

Experiment 2 Partial Vision Analysis: Analysis of Variance Summary Table for Displacement at Time of Peak

Velocity/Target Distance

\begin{tabular}{|c|c|c|c|c|c|c|}
\hline SOURCE & SS & $\mathrm{DF}$ & MS & $\mathrm{F}$ & & $\mathrm{P}$ \\
\hline Blocks/Subjects & 615690 & 9 & & & & \\
\hline Partialvision & 9898 & 1 & 9898 & 3 & 456 & 093 \\
\hline Error & 25778 & 9 & 2864 & & & \\
\hline MixType & 136916 & 2 & $68 \quad 458$ & 2 & 988 & 074 \\
\hline Error & 412400 & 18 & 22911 & & & \\
\hline \multicolumn{7}{|l|}{ Partialvision } \\
\hline $\mathrm{x}$ Mixtype & $28 \quad 504$ & 2 & 14252 & 10 & 487 & 001 \\
\hline Error & $24 \quad 463$ & 18 & 1359 & & & \\
\hline Total & 1253651 & 59 & & & & \\
\hline (Residual) & $462 \quad 641$ & 45 & & & & \\
\hline
\end{tabular}

Table $35 b$

Experiment 2 Partial Vision Analysis: PVDISP/DIST Means and Standard Deviations

Vision Mix No Vision Mix Blocked

$\begin{array}{ccccc}\text { First-Half Vision } & \overline{\mathrm{x}} & 420 & 459 & 462 \\ & \text { SD } & 48 & 33 & 49 \\ \text { Last-Half Vision } & \overline{\mathrm{x}} & 425 & 457 & 435 \\ & \text { SD } & 54 & 34 & 46\end{array}$

